Florida International University

FIU Digital Commons

FIU Electronic Theses and Dissertations

University Graduate School

$11-22-1996$

\title{
Reactions of singlet oxygen and singlet oxygen mimics with dicyclopropylethylenes: comparison of reactivity and mechanisms
}

Gina Elizabeth Alume

Florida International University

DOI: $10.25148 /$ etd.FI13101612

Follow this and additional works at: https://digitalcommons.fiu.edu/etd

Part of the Chemistry Commons

\section{Recommended Citation}

Alume, Gina Elizabeth, "Reactions of singlet oxygen and singlet oxygen mimics with dicyclopropylethylenes: comparison of reactivity and mechanisms" (1996). FIU Electronic Theses and Dissertations. 1216.

https://digitalcommons.fiu.edu/etd/1216 


\section{FLORIDA INTERNATIONAL UNIVERSITY \\ Miami, Florida}

REACTIONS OF SINGLET OXYGEN AND SINGLET OXYGEN MIMICS WITH DICYCLOPROPYLETHYLENES: COMPARISON OF REACTIVITY AND MECHANISMS

A thesis submitted in partial satisfaction of the requirements for the degree of

MASTER OF SCIENCE

IN

CHEMISTRY

b y

Gina Alume

1996 
To: Dean Arthur W. Herriott

College of Arts and Sciences

This thesis, written by Gina Alume, and entitled "Reactions of singlet oxygen and singlet oxygen mimics with dicyclopropylethylenes: Comparison of reactivity and mechanisms" having been approved in respect to style and intellectual content, is referred to you for judgment.

We have read this thesis and recommend that it be approved.

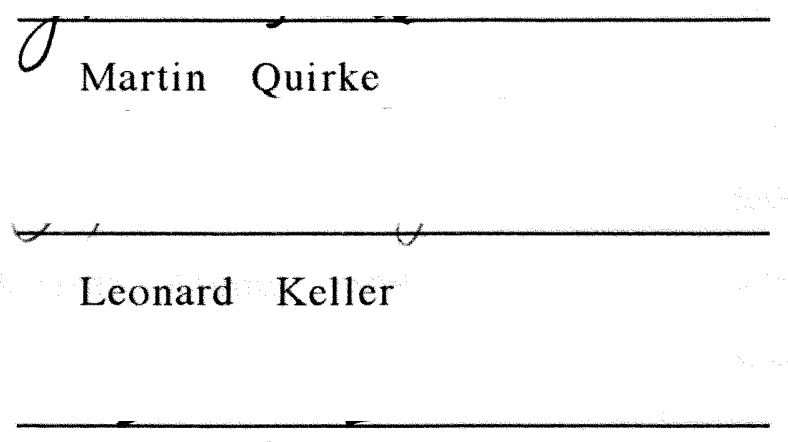

Kevin O'Shea, Major Professor

Date of Defense: November 22nd, 1996

The thesis of Gina Alume is approved.

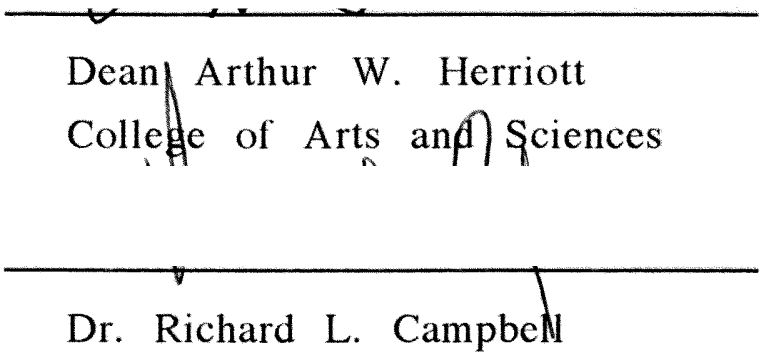

Dean of Graduate Studies

Florida International University, 1996 
COPYRIGHT (C) Gina Alume, 1996

All rights reserved 
I dedicate my thesis to my husband Marcos, my son Anthony and my parents Martha and Ricardo. Without their patience, support, understanding and most of all love, the completion of this work would not have been possible. 


\section{ACKNOWLEDGEMENTS}

I wish to thank the members of my committec Dr. Leonard Keller and Dr. Martin Quirke for their comments. Dr. Ramon Lopez de la Vega and Dr. Stephen Winkle for their assistance with the NMR cxperiments. I want to thank Ms. Zaida Morales-Martinez for her guidance in my teaching assignments; all the professors in the FIU Chemistry Department, for their motivation. I also want to thank Claudia Cardona, John Walton and Stephan Jannach for their unvaluable help during my first year as a graduate student and the members of the lab Ivelitza, Sary, Martha, Ailette, Melita and Sugunya for their friendship and assistance.

A special thanks to my professor, Dr. Kevin E. O'Shea, for his guidance, advise, encouraging comments, and especially for having the confidence in me to give me the chance to do this project. 


\section{ABSTRACT OF THE THESIS}

REACTIONS OF SINGLET OXYGEN AND SINGLET OXYGEN MIMICS WITH DICYCLOPROPYLETHYLENES: COMPARISON OF REACTIVITY AND

MECHANISMS

b y

Gina Alume

Florida International University, 1996

Miami, Florida

Professor Kevin E. O'Shea, Major Professor

An extensive study of the reaction pathways of 1,1dicyclopropyl ethylene, cis- and trans- 1,2-dicyclopropylethylenes has been undertaken with different electrophiles 4-methyl-1,2,4 triazoline-3,5-dione (MTAD), tetracyanoethylene (TCNE), and singlet oxygen $\left({ }^{1} \mathrm{O}_{2}\right)$. Comparison of reactivity and reaction mechanisms among the electrophiles is investigated. Singlet oxygen exhibits significantly lower reactivity compared to the other electrophiles. MTAD and TCNE react with dicyclopropylethylenes to produce predominantly "2+2" adducts and a small amount of the "ene" adducts. The " $2+2 "$ is the major product presumably because of the high activation energy leading to the highly strained "ene" products. Solvent trapping studies provide strong evidence of a "stepwise" mechanism, involving a zwitterionic or aziridinium imide as an intermediate from the study of the reactions products of dicyclopropylethylenes and MTAD. 
I. INTRODUCTION.

1.1 General Bakground....................................................................... 2

1.2 Reaction Mechanisms of 1 On, MTAD, TCNE............................... 4

1.3 Conjugation and Reactivity of

Cyclopropyl Groups.............................................................. 16

II. SYNTHESIS AND CHARACTERIZATION OF DICYCLOPROPYLETHYLENES.

2.1 Synthesis and Characterization of

1,1-dicyclopropylethylene.

2.2 Synthesis and Characterization of cis-1,2-dicyclopropylethylene

2.2.1 Synthesis of cis-1,2-dicyclopropylethylene via Wittig Reaction.. 36

2.2.2 Synthesis of cis-1,2-dicyclopropylethylene by Fritsch-Buttemberg-Wiechell Rearrangement...... 39

2.3 Synthesis and Characterization of trans-1,2-dicyclopropylethylene

2.3.1 Synthesis of trans-1,2-dicyclopropylethylene via Wittig-Horner reaction. 55

2.3.2 trans-Selective olefin synthesis by reversibility of betaine formation during the Wittig reaction.... 56

2.3.3 Synthesis of trans-1,2-dicyclopropylethylene using lithium aluminum hydride, a homogeneous hydrogenation catalyst....

2.4 Synthesis and Characterization of

2,4-dimethyl-3-pentene. 61 
III. REACTION STUDIES AND PRODUCT DISTRIBUTION

3.1 Singlet oxygen reactions with dicyclopropyl

substituted ethylenes

3.1.1 The reaction of 1,1-dicyclopropylethylene

with singlet oxygen

3.1.2 The reaction of cis-dicyclopropylethylene

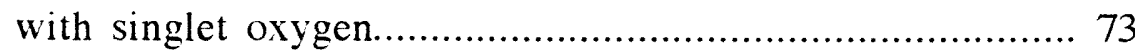

3.1.3 The reaction of 1,1-diisopropylethylene with singlet oxygen.

3.2 Reactions of 4-methyl-1,2,4-triazoline-3,5-dione

(MTAD) with disubstituted ethylenes.

3.2.1 Reaction of 4-methyl-1,2,4-triazoline-3,5-dione

(MTAD) with 1,1-dicyclopropylethylene................ 77

3.2.2 Reaction of 4-methyl-1,2,4-triazoline-3,5-dione

(MTAD) with cis-1,2-dicyclopropylethylene.

3.2.3 Reaction of 4-methyl-1,2,4-triazoline-3,5-dione (MTAD) with trans-1,2-dicyclopropylethylene...

3.2.4 Reaction of 4-phenyl-1,2,4-triazoline-3,5-dione (PTAD) with 1,1-diisopropylethylene.

3.3 Reaction of tetracyanoethylene (TCNE) with dicyclopropyl substituted ethylenes.

3.3.1 Reaction of tetracyanoethylene (TCNE) with 1,1-dicyclopropylethylene.

3.3.2 Reaction of tetracyanoethylene (TCNE) with cis-dicyclopropylethylene. 
Figure I

Figure II

Figure III

Figure IV

Figure V

Figure VI

Figure VII

Figure VIII

Figure IX

Figurc $\mathrm{X}$

Figure XI

Figure XII

Figure XIII

Figure XIV

Figure $\mathrm{XV}$

Figure XVI

Figure XVII

Figure XVIII

Figure XIX
Structure of Elcctrophiles............................................ 6

Non-concerted Reaction Mechanisms...................... 7

Structure of Substrates.............................................. 17

Cyclopropylcarbinyl Cation Equilibrium................ 23

${ }^{1}$ H NMR Spectrum of dicyclopropyl ketonc........... 30

$13 \mathrm{C}$ NMR Spectrum of dicyclopropyl ketone......... 31

${ }^{1} \mathrm{H}$ NMR Spectrum of 1,1-dicyclopropylethylenc.. 32

$13 \mathrm{C}$ NMR Spectrum of 1,1-dicyclopropylethylene 33

Mass Spectrum of 1,1-dicyclopropylethylene....... 34

${ }^{1} \mathrm{H}$ NMR Spectrum of 1-chloro-2,2-

dicyclopropylethylene.

$13 \mathrm{C}$ NMR Spectrum of 1-chloro-2,2-

dicyclopropylethylene.

Mass Spectrum of 1-chloro-2,2-

dicyclopropylethylene.

${ }^{1} \mathrm{H}$ NMR Spectrum of 1,2-dicyclopropylethyne..... 46

$13^{3} \mathrm{C}$ NMR Spectrum of 1,2-dicyclopropylethyne... 47

Mass Spectrum of 1,2-dicyclopropylethyne.......... 48

${ }^{1}$ H NMR Spectrum of cis-dicyclopropylethylene... 50

$1{ }^{3} \mathrm{C}$ NMR Spectrum of cis-dicyclopropylethylene. 51

Mass Spectrum of cis-dicyclopropylethylene........ 52

${ }^{1} \mathrm{H}$ NMR Spectrum of

trans-dicyclopropylethylene .59 
Figure $X X$

$13 \mathrm{C}$ NMR Spcctrum of

trans-dicyclopropylethylene

Figure XXI

${ }^{1} \mathrm{H}$ NMR Spectrum of 2,4-dimethyl-3-pentanone. 63

Figure XXII

${ }^{1} \mathrm{H}$ NMR Spectrum of 1,1-diisopropylethylene $\ldots . .64$

Figure XXII

$13 \mathrm{C}$ NMR Spectrum of 1,1-diisopropylethylenc $\ldots .65$

Figure XXIV

${ }^{1} \mathrm{H}$ NMR Spectrum of the reaction of 1,1 -

dieyclopropylethylene with singlet oxygen

in the presence of tetramethylene.

71

Figure XXV

$1{ }^{3} \mathrm{C}$ NMR Spectrum of the reaction of 1,1 -

dicyclopropylethylene with singlet oxygen

in the presence of tetramethylene.

72

Figure XXVI ${ }^{1} \mathrm{H}$ NMR Spectrum of reaction of 1,1 -

dicyclopropylethylene with MTAD.

Figure XXVII 13 C NMR Spectrum of reaction of 1,1 -

dicyclopropylethylene with MTAD.

81

Figure XXVIII Attached Proton Test Spectrum of reaction of 1,1-

dicyclopropylethylene with MTAD..................... 82

Figure XXIX $\quad{ }^{1} \mathrm{H}$ NMR Spectrum of cycloaddition product of

reaction of 1,1-dicyclopropylethylene

with MTAD.

84

Figure XXX Mass Spectrum of cycloaddition product of

reaction of 1,1-dicyclopropylethylene

with MTAD.

Figure XXXI ${ }^{1} \mathrm{H}$ NMR Spectrum of ene product of reaction of

1,1-dicyclopropylethylene with MTAD................ 86 
Figure XXXII Mass Spectrum of enc product of reaction of 1,1-dicyclopropylethylene with MTAD................ 87

Figure XXXIII ${ }^{1} \mathrm{H}$ NMR Spectrum of reaction of 1,1 dicyclopropylethylene with MTAD in the presence of methanol.................................... 91

Figure XXXIV ${ }^{3} \mathrm{C}$ NMR Spectrum of reaction of 1,1 dicyclopropylethylene with MTAD in the presence of methanol.................................. 92

Figure XXXV Attached Proton Test Spectrum of reaction of 1,1-dicyclopropylethylene with MTAD in the presence of methanol...................................... 93

Figure XXXVI ${ }^{1} \mathrm{H}$ NMR Spectrum of reaction of cisdicyclopropylethylene with MTAD...................... 96

Figure XXXVII $13 \mathrm{C}$ NMR Spectrum of reaction of $c$ is dicyclopropylethylene with MTAD........................ 97

Figure XXXVIII Attached Proton Test Spectrum of reaction of cis-dicyclopropylethylene with MTAD................. 98

Figure XXXIX Coupling constant of diazetidine of reaction of cis-dicyclopropylethylene with MTAD................... 99

Figure XL $\quad{ }^{1} \mathrm{H}$ NMR Spectrum of reaction of $c$ isdicyclopropylethylene with MTAD in the presence of methanol... 102

Figure XLI ${ }^{13} \mathrm{C}$ NMR Spectrum of reaction of $c$ isdicyclopropylethylene with MTAD in the presence of methanol 103 
Figure XLII

Attached Proton Test Spectrum of reaction of cis-dicyclopropylethylene with MTAD

in the presence of methanol.................................. 104

Figure XLIII ${ }^{1} \mathrm{H}$ NMR Spectrum of methanol adduct of

reaction of cis-dicyclopropylethylene

with MTAD

Figure XLIV ${ }^{1} \mathrm{H}$ NMR Spectrum of methanol adduct of

reaction of cis-dicyclopropylethylene with

MTAD in the presence of chiral reagent.............. 106

Figure XLV $\quad 1_{\mathrm{H}}$ NMR Spectrum of reaction of 1,1 -

diisopropylethylene with PTAD

in the presence of methanol..................................... 110

Figure XLVI ${ }^{1} \mathrm{H}$ NMR Spectrum of reaction of 1,1 -

dicyclopropylethylene with TCNE......................... 114

Figure XLVII 13 C NMR Spectrum of reaction of 1,1 -

dicyclopropylethylene with TCNE......................... 115

Figure XLVIII Attached Proton Test Spectrum of reaction

of 1,1 dicyclopropylethylene with TCNE.............. 116

Figure XLIX Mass Spectrum of reaction of 1,1 -

dicyclopropylethylene with TCNE......................... 117

Figure L ${ }^{1} \mathrm{H}$ NMR Spectrum of reaction of cis-

dicyclopropylethylene with TCNE........................ 119

Figure LI $\quad 1^{3} \mathrm{C}$ NMR Spectrum of reaction of cis-

dicyclopropylethylene with TCNE......................... 120 
CHAPTER I

INTRODUCTION 


\subsection{GENERAL BACKGROUND}

The oxidation of organic and biological substrates in the presence of oxygen, light and a photosensitizer has been the subject of extensive study over the past several decades.1,2 This photooxidation requires a number of steps. Upon absorption of a photon of light, the sensitizer is excited to a singlet state $\left({ }^{1} \mathrm{~S}\right)$ which undergoes rapid intersystem crossing to a triplet excited state $\left({ }^{3} \mathrm{~S}\right)$. The triplet state, which is generally longer lived than the $1 \mathrm{~S}$, can transfer its energy to triplet oxygen $\left({ }^{3} \mathrm{O}_{2}\right)$ in a spin-allowed process to form singlet oxygen $\left({ }^{1} \mathrm{O}_{2}\right)$, or it can undergo electron transfer to initiate a radical oxidation process.

Singlet oxygen $\left({ }^{1} \Delta_{\mathrm{g}}\right)$ is the lowest electronic excited state of molecular oxygen, $22.4 \mathrm{Kcal} / \mathrm{mole}$ above the ground state. It is short lived in solution with a lifetime of $10^{-3}-10^{-6}$ seconds depending on solvent and experimental conditions.

Singlet oxygen is involved in a number of chemical, biological and environmental processes, and thus its properties and reactions are of great interest to a variety of scientists. Several techniques have been developed which employ photooxidative processes for a variety of applications, from the treatment of skin disorders to the destruction of pollutants. The use of a photosensitizer in the presence of oxygen (Photodynamic Therapy, PDT) for the treatment of earlystage superficial tumors 3 is one of the new advances in the medical field involving photooxidation. A fundamental understanding of the reaction pathways and intermediates involved in these processes is 
crucial for the application and implementation of PDT and other photooxidative technologies.

The reaction mechanisms involved in photosensitized oxidation can be characterized as Type I or Type II (Scheme I). ${ }^{4}$ In the Type I process, substrate, solvent or oxygen reacts with the photoexcited sensitizer to give radical or radical ions, by hydrogen atom or electron transfer. These radicals commonly react with oxygen to give oxygenated products. In the Type II process, the photoexcited sensitizer transfers energy to ground state oxygen to form singlet molecular oxygen $\left({ }^{1} \mathrm{O}_{2}\right)$ which can react with a variety of substrates to form oxygenated products. The Type I and Type II mechanisms are in competition, the factors governing the competition are oxygen concentration, reactivities of the substrates and photoexcited sensitizer, substrate concentration, and singlet oxygen lifetime. 5

Scheme I: Mechanism of Photooxygenation

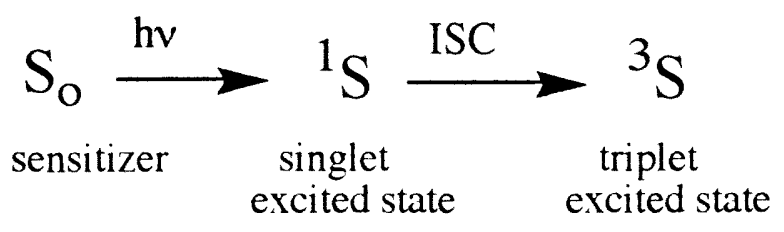

"TYPE I"

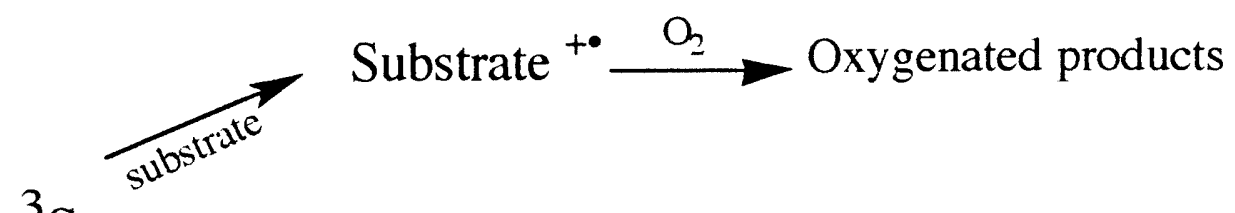

$3 \mathrm{~S}$

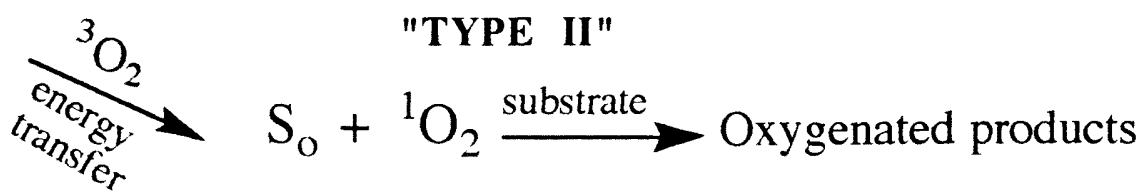




\subsection{REACTION MECHANISMS OF $1 \mathrm{O}_{2}$, MTAD, TCNE.}

We have been interested in the reactions of singlet oxygen with unsaturated systems, particularly alkenes, because $\mathrm{l}_{\mathrm{O}}$ can lead to the damage of biological target molecules with the consequent biochemical effects that include enzyme deactivation, nucleic acid oxidation and membrane damage, also degradation of polymeric materials such as rubber and plastics.

Singlet oxygen reacts with olefins in a "2+2" type cycloaddition affording a dioxetane, which generally cleaves to give two carbonyl compounds accompanied by chemiluminescence, as shown below.

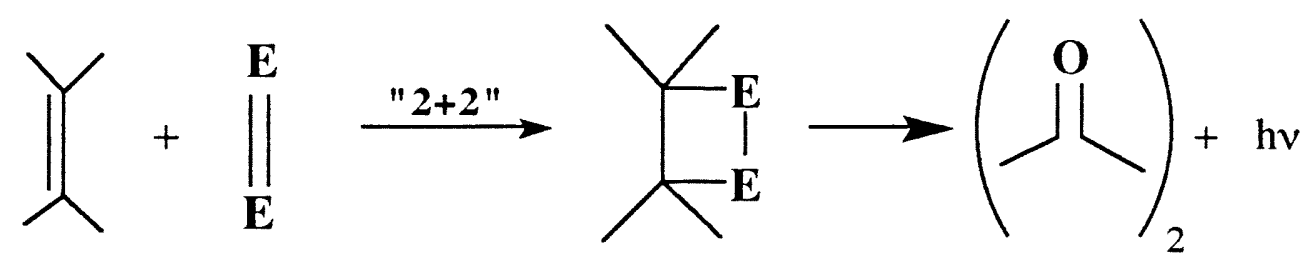

DIOXETANE $\left({ }^{1} \mathrm{O}_{2}\right)$

$\mathrm{E}=\mathrm{E}=\mathrm{l}_{\mathbf{O}}$

An "ene" type reaction can occur with alkenes with allylic hydrogens to afford a hydroperoxide. Due to its instability, 6 the hydroperoxide is usually reduced to the corresponding alcohol prior to its isolation and identification. Singlet oxygen can also react with dienes in a [4+2] cycloaddition to give an endoperoxide as its product, as shown below. 


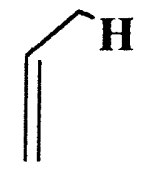

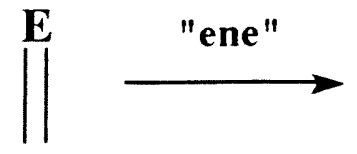

$\mathbf{E}$<smiles>C=C[Te]C=C</smiles>

HYDROPEROXIDE $\left({ }^{1} \mathrm{O}_{2}\right)$

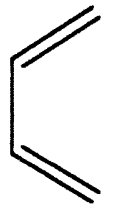

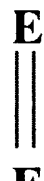

$\mathbf{E}$

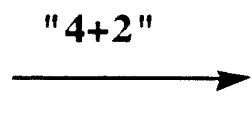

\section{ENDOPEROXIDE $\left({ }^{1} \mathrm{O}_{2}\right)$}

$\mathrm{E}=\mathrm{E}=1_{\mathrm{O}_{2}}$

Several reports ${ }^{7}$ suggest that $\mathrm{TCNE}$ and $\mathrm{RTAD}^{\mathrm{N}}$ undergo reactions similar to those of $1 \mathrm{O}_{2}$, but they produce more stable products which are readily isolated and identified under moderate conditions. We have chosen to study the reactions of 4-alkyl-1,2,4-triazoline-3,5dione (RTAD), tetracyanoethylene (TCNE), and ${ }^{1} \mathrm{O}_{2}$ with alkenes (Figure I). The two former compounds are highly reactive and well known as diagnostic reagents for the study of cycloaddition reactions of acyclic and cyclic olefins. RTAD reacts with olefins in an ene type reaction to give $\mathrm{N}$-allylurazoles, and with some olefins in a "2+2" type reaction to afford 1,2-diazetidines. 


\section{Figure I: Structure of Electrophiles}
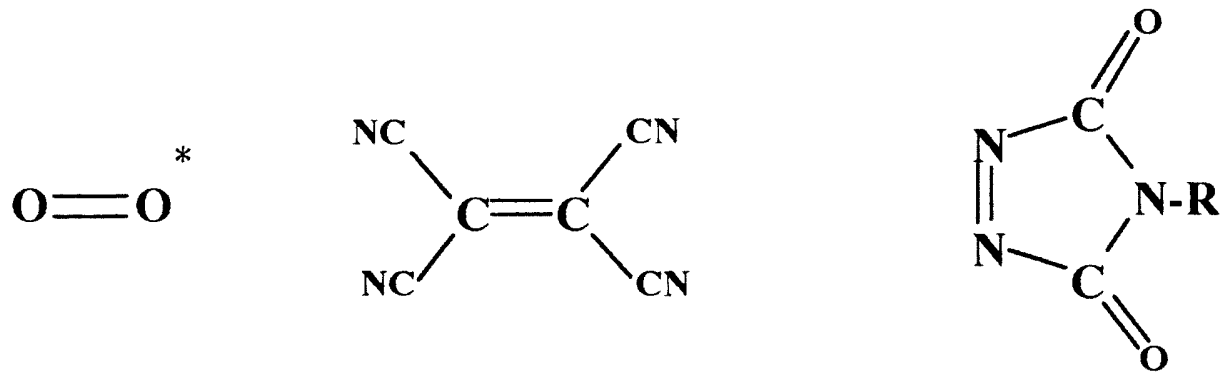

Singlet Oxygen Tetracyanoethylene<smiles>[18OH]</smiles>

TCNE

4-Alkyl -1,2,4-Triazoline-

3,5-Dione

RTAD

$\mathbf{R}=\mathbf{M e}, \mathbf{P h}$

Although the reactions of olefins and aromatics with the electrophile, ${ }^{1} \mathrm{O}_{2}, \mathrm{RTAD}$ and TCNE, have received considerable attention recently, there are still controversy and uncertainty regarding the mechanistic details of these reactions. Strong evidence for both concerted and non-concerted mechanisms has been reported. 8

The observation and trapping of unstable intermediates in the reaction of MTAD, generally the most reactive of the three dienophiles, with olefins have been reported.9,10 Kinetic isotopic effects and product studies suggest that reaction of olefins with RTAD and $\mathrm{IO}_{2}$ involve intermediates with similar geometry, a close zwitterion, called aziridinium imide (AZI) if the electrophile is RTAD and perepoxide if the electrophile is ${ }^{1} \mathrm{O}_{2}$ (Figure II). 
Figure II: Non-concerted Reaction Mechanisms

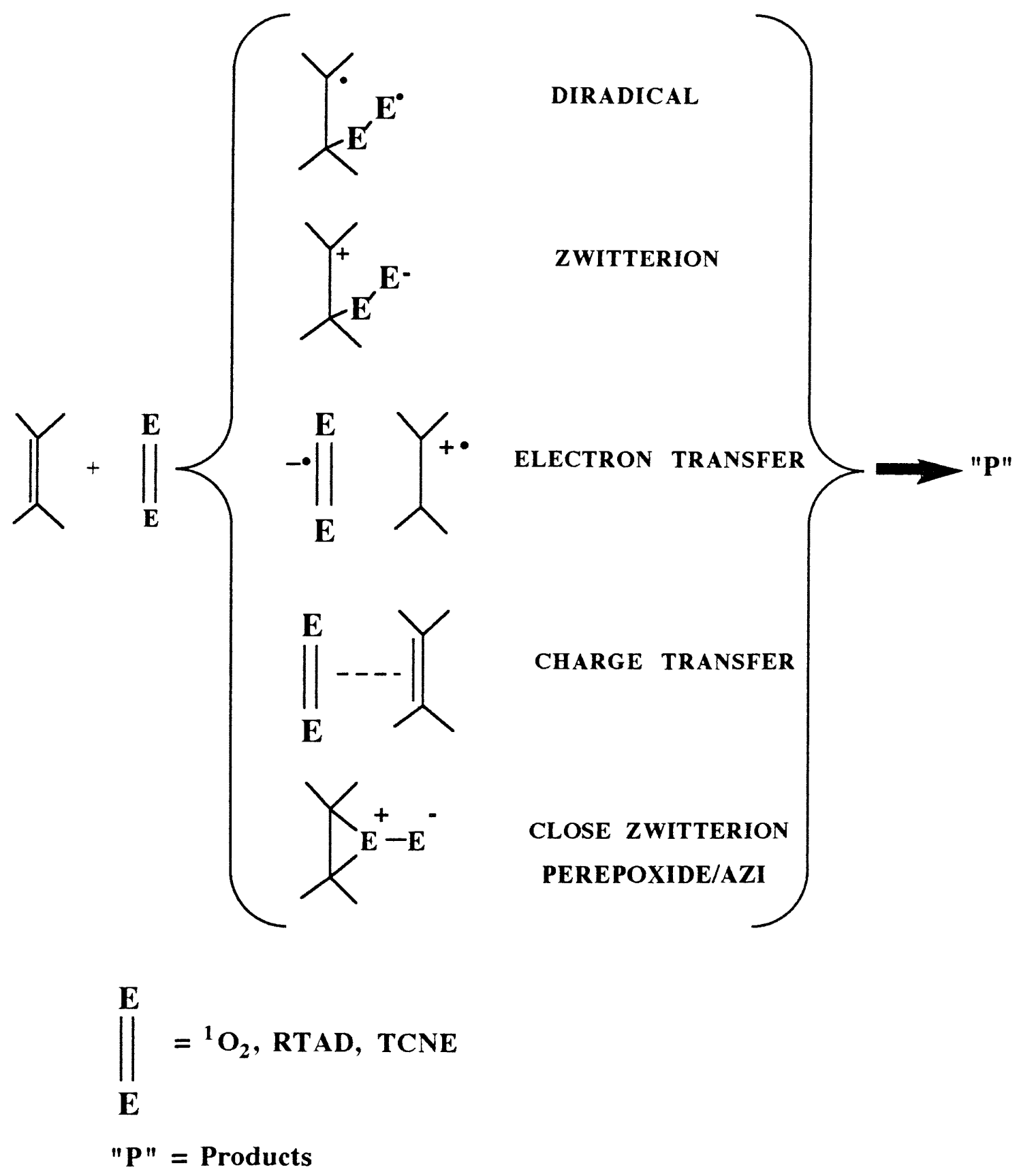


The debate between concerted and non-concerted mechanisms for these electrophilic reactions has received substantial attention because of the strong experimental evidence indicating an intermediate present in some of the reaction pathways. Recent reports provide strong support that some of these reactions involve non-concerted pathways. A number of non-concerted mechanisms involving 1,4-biradicals, ${ }^{11}$ perepoxides, 12 1,4-zwitterions, ${ }^{13}$ charge transfer, and electron transfer has bcen proposed for these types of reactions, Figure II.

Although the reaction mechanisms appear to depend on the substrate, strong evidence for dipolar intermediates in reactions of olefins with $1 \mathrm{O}_{2}$ and the singlet oxygen mimics, RTAD and TCNE, has been reported. A number of systems in which formation of a carbocation stabilized by conjugation with a double bond or aromatic system provides convincing evidence for zwitterionic intermediates. Jefford ${ }^{1+}$ has trapped zwitterionic intermediates during the addition of 2-methoxynorbornene to $\mathrm{I}_{2}$, which is complete in 30 minutes. This intermediate is sufficiently long-lived to be trapped by alcohols and aldehydes. In aprotic solvents, only the exo dioxetane and its cleavage product were obtained. However, in protic solvents a mixture of the exo dioxetane, its cleavage product, and the hydroperoxyketal is afforded. In the presence of methanol, oxygenation is slower, giving the cleavage product of exo dioxetane together with the corresponding ketal and a condensation product in substantial amounts, shown below. 


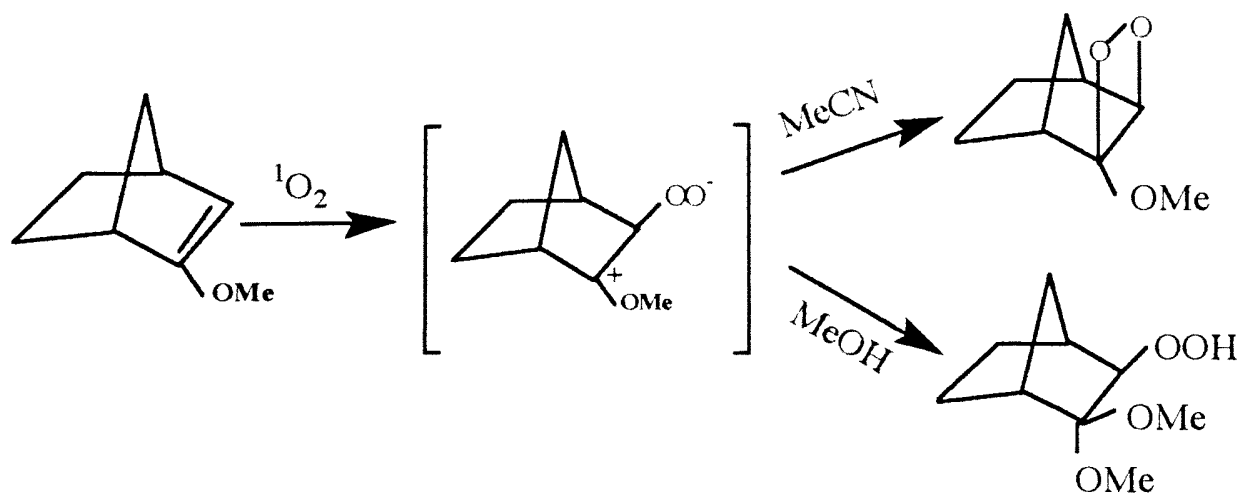

Jensen and Foote 15 investigated the reactions of substituted butadienes with PTAD in the absence and presence of methanol. $(\mathrm{Z}, \mathrm{Z})$-Hexadiene reacts with PTAD to afford a mixture of two DielsAlder products; the major product is non-stereospecific. In addition, the formation of methanol adducts further supports the involvement of a non-concerted pathway and the presence of a polar, trappable intermediate. Initial formation of an aziridinium imide that subsequently opens to a 1,4-zwitterion is proposed.

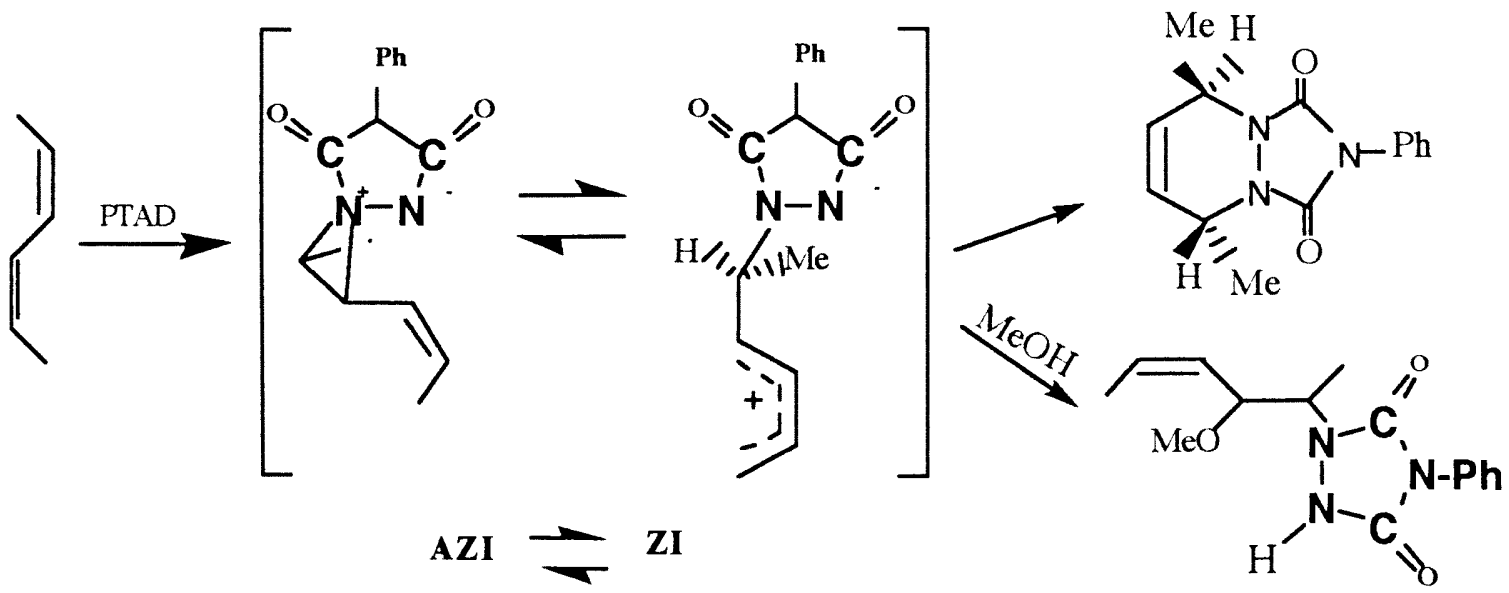


O'Shea and Foote ${ }^{16}$ studied the photooxidation of the three isomeric 2,4-hexadienes at $-78{ }^{\circ} \mathrm{C}$ reporting evidence for a zwitterionic intermediate analogous to the Jensen study. ${ }^{15}$ These dienes react with singlet oxygen affording endoperoxides as the major products, a mixture of hydroperoxides, acetaldehyde and cisand trans-2-butenal. The non-stereospecific product distribution requires the involvement of an open zwitterionic intermediate that can undergo rotation at the former double bond. Although an equilibrium between open and closed zwitterions is postulated as shown below, attempts to characterize the zwitterionic intermediate by low temperature NMR were unsuccessful.

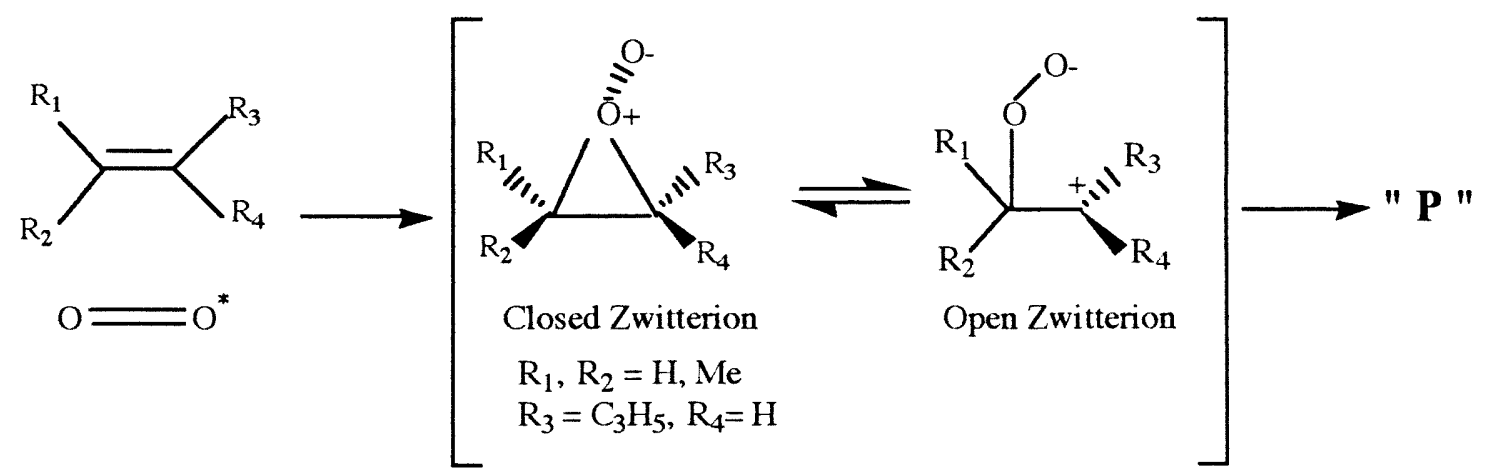

Sharp ${ }^{17}$ was the first to suggest a perepoxide intermediate. Kearns ${ }^{18}$ isolated trapping products consistent with a perepoxide intermediate. Frimer and Barlett ${ }^{19}$ studied the isotope effect in some dihydropyrans and proposed the involvement of a perepoxide or a charge-transfer complex with similar geometry, to yield both ene and " $2+2$ " products. 


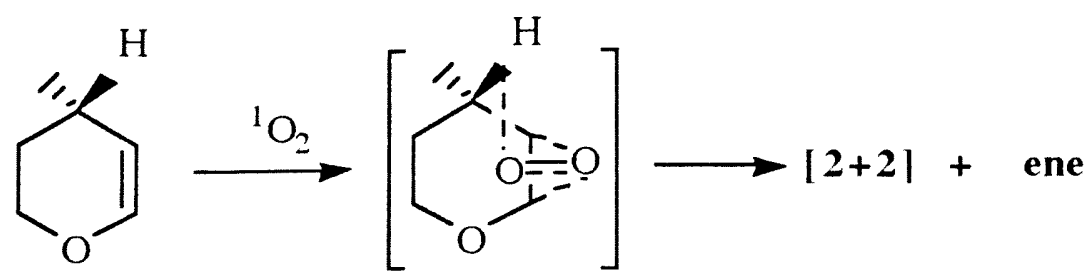

Stephenson 20 and Greene 21 used kinctic isotope effects to provide strong evidence for the perepoxide and aziridinium imide $(A Z I)$ in the "ene" type reaction of $1 \mathrm{O}_{2}$ and RTAD with (Z)- and (E)2,3-bis(trideuteriomethyl)-2-butene. Greene observed an isotope effect only when $\mathrm{CH}_{3}$ is cis to $\mathrm{CD}_{3}, \mathrm{k}_{\mathrm{H}} / \mathrm{k}_{\mathrm{D}}=3.8, \mathrm{R}=\mathrm{CH}_{3}, \mathrm{k}_{\mathrm{H}} / \mathrm{k}_{\mathrm{D}}=3.7$, $\mathrm{R}=\mathrm{Ph}$. In contrast, when $\mathrm{CH}_{3}$ is trans or geminal to $\mathrm{CD}_{3}, \mathrm{k}_{\mathrm{H}} / \mathrm{k}_{\mathrm{D}}=1.08$ $\mathrm{R}=\mathrm{CH}_{3}, \mathrm{k}_{\mathrm{H}} / \mathrm{k}_{\mathrm{D}}=1.1 \mathrm{R}=\mathrm{Ph}$. These results imply the involvement of an intermediate which is polarized to one side (cis effect). A representation of an intermediate in which little or no breaking of an allylic carbon-proton bond has taken place is the aziridinium imide, AZI, analogous to the perepoxide, shown below.

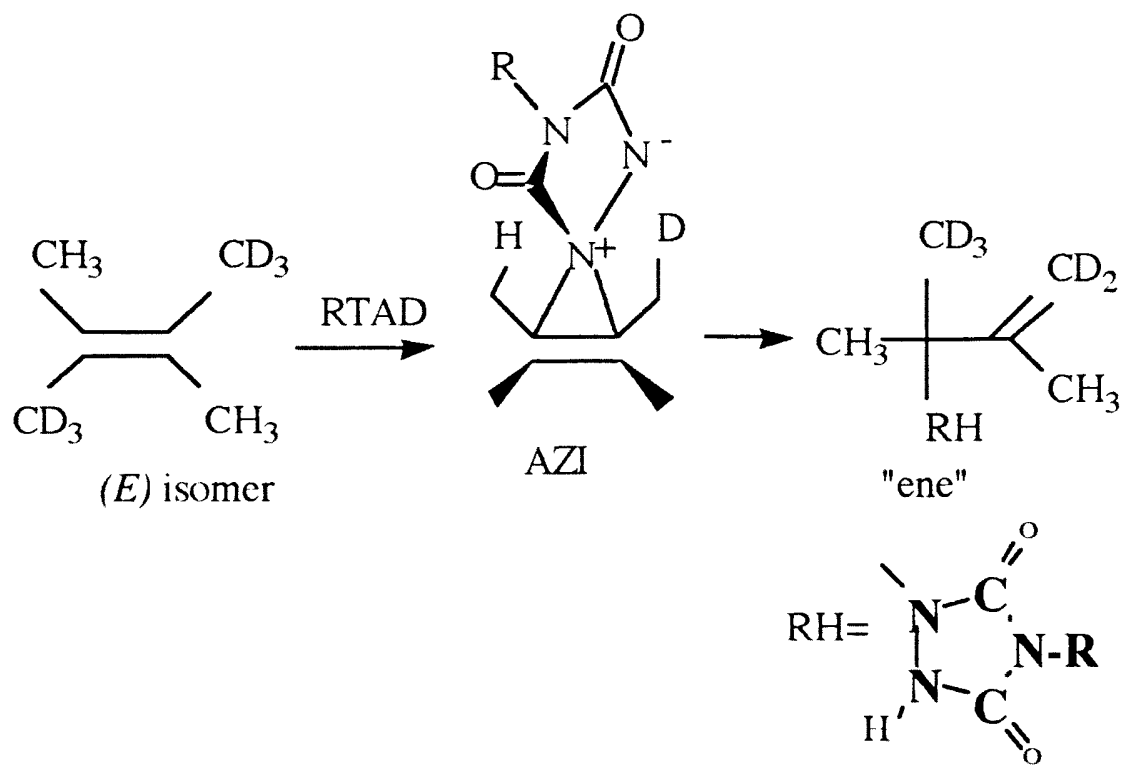


A limited number of investigators have reported the direct observation of aziridinium imide, AZI. Nelsen22 observed the AZI during the "2+2" cycloaddition reaction of adamantylideneadamantane and 4-phenyl-1,2,4-triazoline-3,5-dione (PTAD) by ${ }^{1}$ H-NMR. The FT-IR spectrum of the intermediate shows carbonyl absorptions at 1790 and $1686 \mathrm{~cm}^{-1}$ consistent with expectations based on an ammonium imide model, with carbonyl absorptions reported to be at 1790 and $1670 \mathrm{~cm}^{-1}{ }^{1} \mathrm{H}-$ and $13 \mathrm{C}-\mathrm{NMR}$ were used to follow the dissappearance of the reactants and the formation of an intermediate, presumably aziridinium imide, AZI.

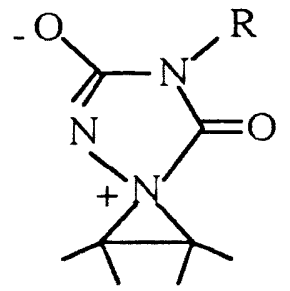

AZIRIDINIUM IMIDE

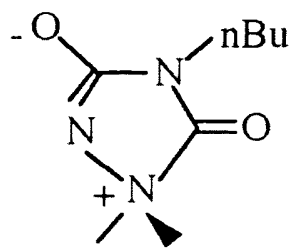

AMMONIUM IMIDE

Squillacote 23 showed spectral evidence of an AZI at $-135^{\circ} \mathrm{C}$ and its conversion to the "ene" product at higher temperatures in the ene reaction of trans-cycloheptene and 4-methyl-1,2,4-triazoline3,5-dione, shown below.

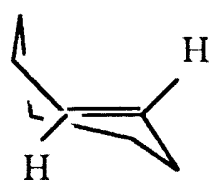

MTAD

$\mathrm{H}$

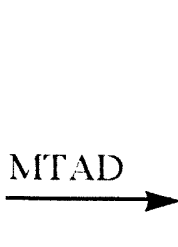

trans-Cy cloheptene

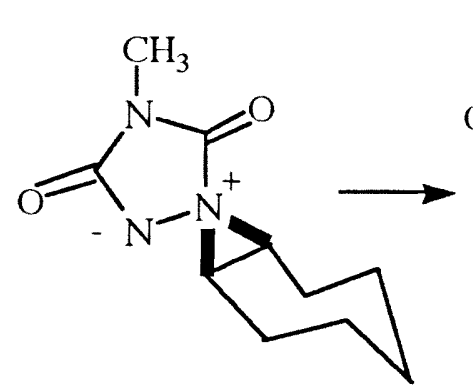

( A Z I)

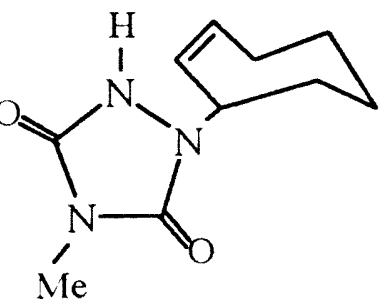

e n e - p rod u c t 
Orfanopoulos and coworkers ${ }^{2+}$ reported the addition and stereochemistry of methanol/PTAD adducts formed in the reaction of PTAD with cis- and trans-2-butene, 1-methylcyclopentene, (E)-2methyl-2-butene-1,1,1-d3 and substituted indenes (indene, 2methylindene, 2,3-dimethylindene). There is no loss of stereochemistry in the addition of methanol and PTAD to most of these compounds although methyl-substituted indenes lose their stereochemistry at the reaction center. The preference for open and closed zwitterion intermediates is established from the stereospecific methanol adducts. A strong directing effect in the ene reaction toward attack at the crowded side in the substituted olefin (Markonikov effect) is observed. It is only in benzylically-stabilized tertiary indenes, however, that the zwitterionic intermediate is stable enough to observe loss of stereochemistry in the methanol adducts.<smiles>CC1=CCc2ccccc21</smiles>

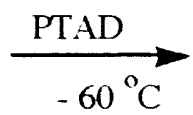

2-methylindene

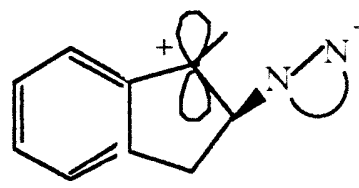

ZI
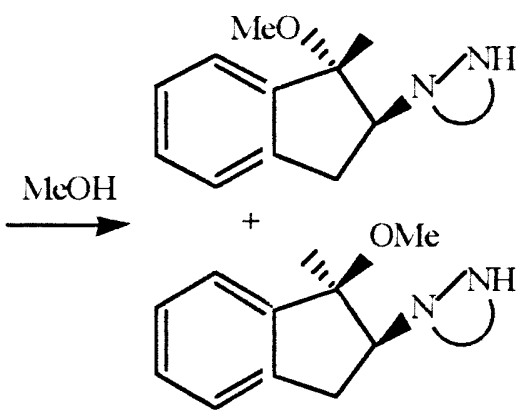

Foote and Poon 25 directly observed an AZI in the reaction of $\mathrm{N}$ substituted-triazolines-3,5-dione and trans-cyclooctene. The reaction occurs stereospecifically affording three different products. Nucleophilic trapping of the intermediate with methanol and water 
suggests an AZI followed by an open zwitterion that can lead to trans-annular ring closure and hydride shifts, as shown below. The formation of a trans-aziridinium imide intermediate at $-83^{\circ} \mathrm{C}$ is observed by low temperature NMR, and its formation was found to be nearly quantitative.

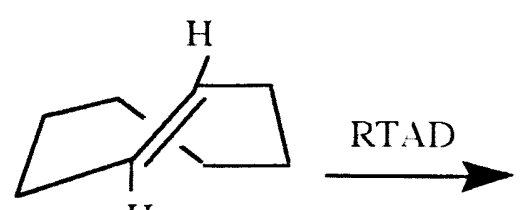

$\mathrm{H}$
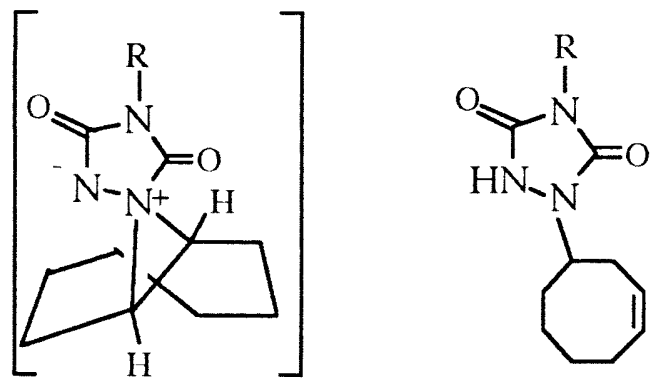

$\mathrm{MeOH}$
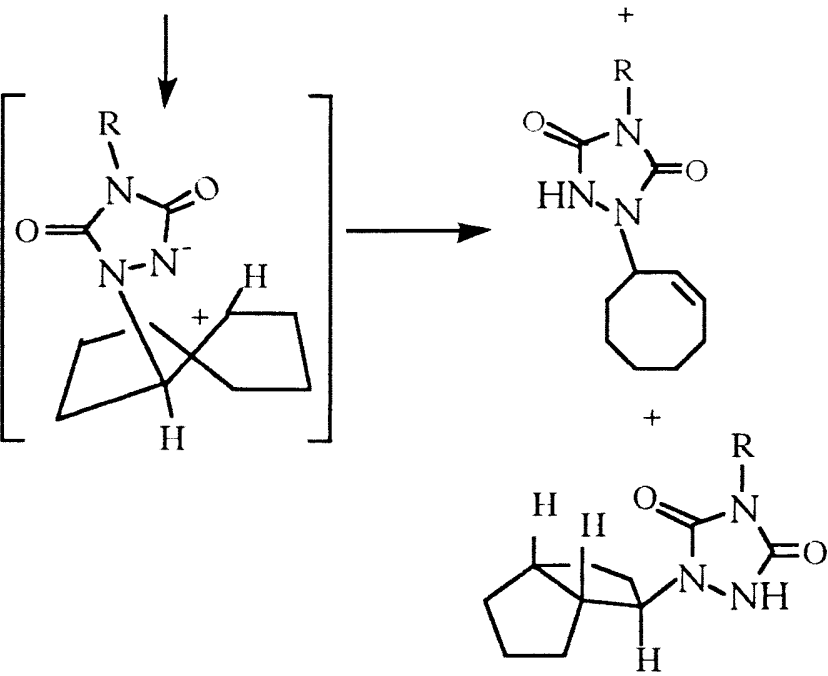

The similarity in the structures of perepoxide and aziridinium imide intermediates and the possibility of using the knowledge obtained from the AZI reactions as applied to the perepoxide chemistry are one of the goals in this study.

Fukui 26 has suggested a geometry for complexes of singlet oxygen with alkenes that is very similar to that of the expected 
perepoxide. Semiempirical MINDO/3 calculations by Dewar and Thiel27 suggest that addition of singlet oxygen to propene to form a perepoxide is $16 \mathrm{kcal} / \mathrm{mol}$ exothermic.

Orbital correlations diagrams 28 and $\mathrm{CNDO} / 2-\mathrm{CI}$ calculations 26 suggest evidence against the formation of the perepoxide. Harding and Goddard 29 favor a diradical intermediate by using GVB-CI calculations which place the perepoxide at least $8 \mathrm{kcal} / \mathrm{mol}$ above the known activation enthalpies.

TCNE, a powerful electrophile, undergoes cycloaddition with olefins and dienes analogous to the products observed with singlet oxygen and RTAD. The reactions of TCNE appear to involve the initial formation of a charge transfer complex. Evidence supporting a polar intermediate has not been reported, such as the AZI and perepoxide.

Nishida, Moritani and Teraji30 investigated the cycloaddition reactions of 1,1-dicyclopropylethylene and cis- and trans-1,2dicyclopropylethylenes with tetracyanoethylene. They suggest the presence of a 1,4-dipolar intermediate in the reaction pathway. The higher reactivity of 1,1-dicyclopropylethylene is attributed to the presence of the geminal cyclopropyl groups that increases the electron density at the double bond in the olefin.

Based on the relative reactivities of tri- and tetracyclopropylethylenes with TCNE, Tsuji and Nishida ${ }^{31}$ suggest an initial electron transfer that affords radical ion pairs. For trisubstituted ethylenes, they observed that the presence of a third cyclopropyl group decreases the rate of the reaction, and an even slower rate is observed by substitution with an isopropyl group. The 
difference in reactivities between the isopropyl and the cyclopropyl group is attributed to electronic effects and to steric hinderance which inhibits the access of TCNE to the $\pi$-bond. The cyclic group is less sterically-hindered than the isopropyl group.

The high reactivity of TCNE towards electron-rich systems and the stabilizing effect of the cyclopropyl group with an adjacent electron-deficient center, motivated us to study the reactions of dicyclopropylethylenes with this electrophile and to compare our results with reports from previous investigations.

\subsection{CONJUGATION AND REACTIVITY OF CYCLOPROPYL GROUPS}

The cyclopropyl groups in 1,1-dicyclopropylethylene and cisand trans-dicyclopropylethylene (Figure III) were used as probes to investigate both their reaction modes with electrophiles and the resulting product distributions. A cyclopropyl group can effectively conjugate with an unsaturated system, similar to an olefinic group. It is also susceptible to attack by electrophiles and undergoes nucleophilic ring opening when the ring is activated by strongly electron withdrawing groups. They can stabilize a carbocation and rearrange into a five-membered ring. 
Figure 11I: Structure of Substrates

1, 1-Dicyclopropylethylene, cis-Dicyclopropylethylene, and trans-Dicyclopropylet hylene.
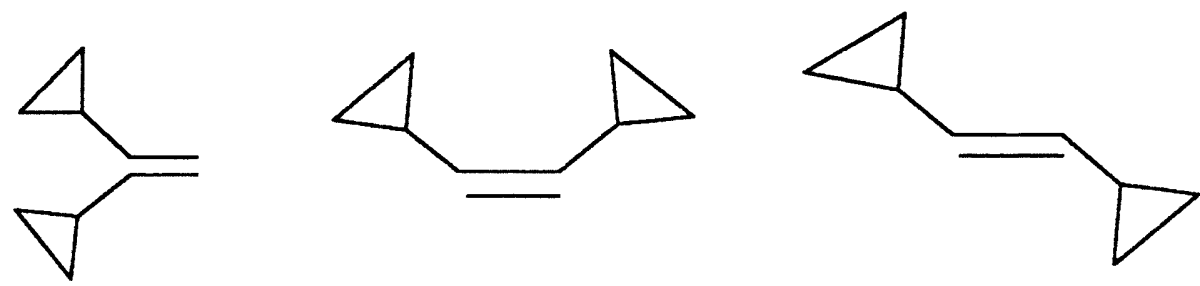

The bonding regions in cyclopropane are easily recognized as the region of positive deformation density between the nuclei, which lies outside of lines drawn between carbons. Such deformation leads to the formation of bent bonds as shown below. In ordinary compounds the deformation density between a pair of bonded carbons is collinear with the conventional bond.

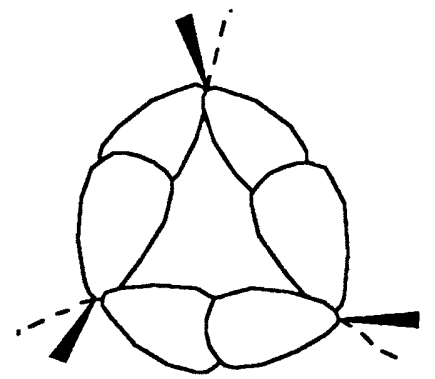

Ingold,32 first proposed the idea that strongly-bent bonds lead to relatively poor overlap and the resulting weak $\mathrm{C}-\mathrm{C}$ bonds in the cyclopropyl group. Theoretically, Forster ${ }^{32}$ recognized the bent character of the $\mathrm{C}-\mathrm{C}$ bond in eyclopropane. Trying to provide an 
explanation for the unusual properties and reactivity of cyclopropanes relative to other saturated compounds. Coulson and Moffit ${ }^{33}$ developed in more detail a zeroth order model starting with $\mathrm{sp}^{3}$-hybridized carbons. From these hybrid orbitals a two-clectron molecular orbital can be readily constructed. Their results confirm the weakness of the C-C bond, the decrease in the s-character of the $\mathrm{C}-\mathrm{C}$ bond and a consequent increase in s-character of the $\mathrm{C}-\mathrm{H}$ bond. These all lead to less bent and stronger bonds with a subsequent increase in the $\mathrm{HCH}$ bond angle. Recent results report a 20\% $\mathrm{s}$ and $80 \%$ p-character for the C-C bonds and $30 \% \mathrm{~s}$ and $70 \%$ p-character for the $\mathrm{C}-\mathrm{H}$ bonds.

Walsh ${ }^{3+}$ proposed a zcroth order model that uscs sp $^{2}$-hybrid orbitals to form the $\mathrm{CH}$ bonds. The $\mathrm{C}-\mathrm{C}$ bonds are formed using $\mathrm{p}$ orbitals as well as $\mathrm{sp}^{2}$ orbitals. The $\mathrm{sp}^{2}$ orbitals point toward the center of the three carbon unit, and the three $p$ orbitals are coplanar. This model also predicts the bent bonds as shown below.

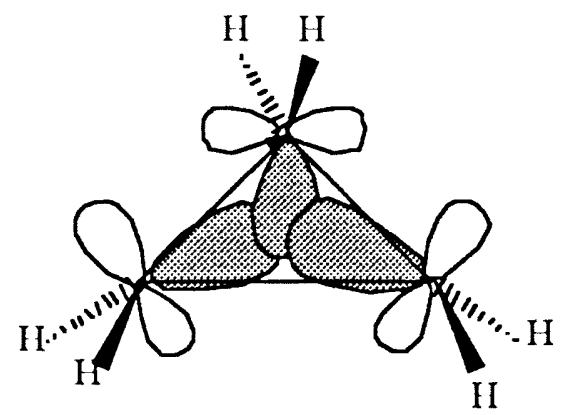

The Walsh description is insufficient for the ground state of cyclopropane and is not equivalent to the Forster-Coulson-Moffit 
model. The degenerate pair of highest occupied molecular orbitals from the Walsh model for cyclopropane is shown bclow.
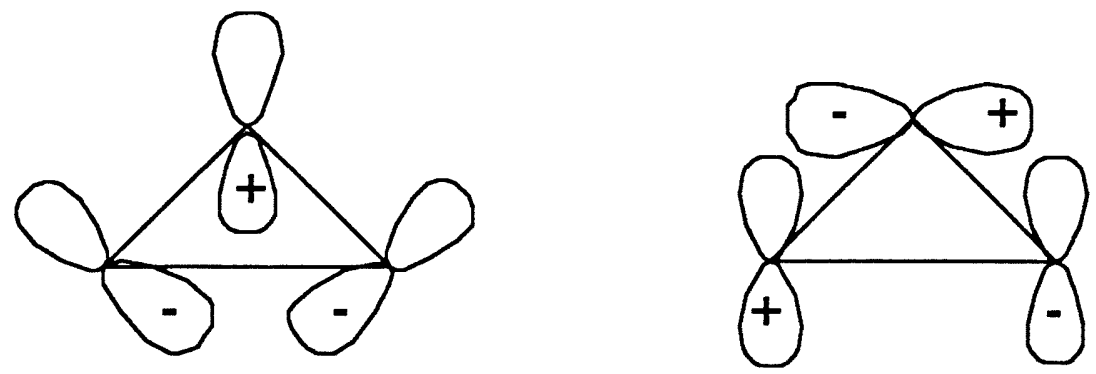

Determination of the nature of the bond from the charge density has been developed by Bader defining which pair of atoms is joined by chemical bonds and the nature of the bond thus formed. 32 His approach uses a characteristic of all chemical bonds which is the bond critical point at which the electron density is a minimum along the path between the nuclei and is a maximum in all directions perpendicular to the path. The path between the nuclei which contains the bond critical point and maximizes the charge density throughout its length is the bond path.

The calculated C-C distance is $1.497 \AA$. The length along the bond path is $1.507 \AA$, a distance which is greater than the straight line connecting the bonded atoms. The C-C-C bond angle, the angle between the bond paths, is $78.84^{\circ}$ rather than the $60^{\circ}$ angle between the lines of centers. The $\mathrm{C}$-C bond order for cyclopropane is 0.99 based on the charge density at the bond critical point that is used as an indicator of the bond order. ${ }^{32}$

Cyclopropyl groups when attached to a $\pi$-system can be good $\pi$ electron donors. 35 Experimental and theoretical studies have shown 
that these $\pi$-donor properties are conformation-dependent. Conjugation and stcric effects play an important rolc in our study because the preferred conformation adopted by the cyclopropyl group will increase or decrease the rate of the reaction and canaffect the product distribution. The overlap of the highest occupied molecular orbital (HOMO) of the cyclopropyl group and the lowest unoccupied molecular orbital (LUMO) of an adjacent $\pi$-system is maximal when the orbitals are coplanar (bisected conformation) 36,37 as shown below.

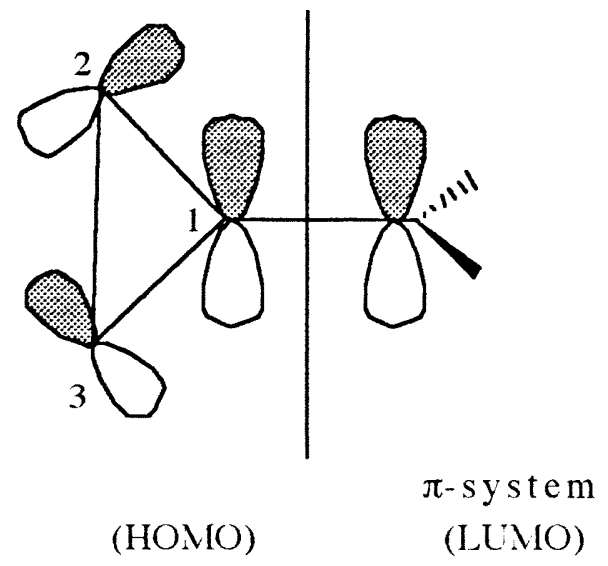

The degree of interaction between the cyclopropyl group and the $\pi$-system in the bisected conformation is indicated by the lengths of the vicinal $\left(\mathrm{C}_{1}-\mathrm{C}_{2}, \mathrm{C}_{1}-\mathrm{C}_{3}\right)$ and distal bond $\left(\mathrm{C}_{2}-\mathrm{C}_{3}\right)$ of the cyclopropyl ring. Transfer of electron density from the HOMO orbital decreases both the bonding character between $\mathrm{C}_{1}-\mathrm{C}_{2}, \mathrm{C}_{1}-\mathrm{C}_{3}$ and the antibonding character between $\mathrm{C}_{2}-\mathrm{C}_{3}$ resulting in a lengthening of the vicinal bonds and a shortening of the distal bond. 
In the perpendicular conformation, the HOMO of the eyclopropyl group is orthogonal to the LUMO of the $\pi$-system. Thus overlap between the orbitals is minimal. For the perpendicular conformation, the cyclopropyl group can function as a weak $\pi$ acceptor, and donation of electron density from the HOMO of the $\pi$ system to the LUMO of the cyclopropyl group is predicted to result in the lenghtening and shortening of the vicinal and distal bonds, respectively, as shown below. This type of stabilization of cyclopropane by $\pi$-donors is expected to be important only for potent $\pi$-donors. 38

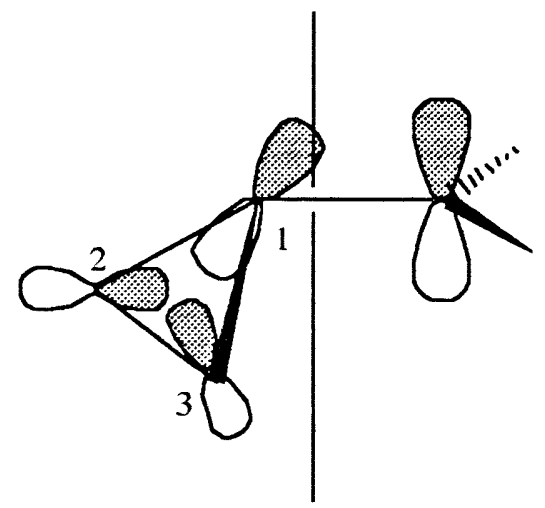

(LUMO)

ח-SYSTEM

(HOMO)

Shimazaki ${ }^{39}$ observed that the cyclopropyl group rearranges because of a high migratory aptitude. Nakamura, 39 found in the pinacol rearrangement of the protonated 1-cyclopropyl-1,2ethanediol the migration of the cyclopropyl group is favored over four other possible pathways. The activation energy for migration of the cyclopropyl group $(17.0 \mathrm{kcal} / \mathrm{mol})$ is lower than for the migration of the methyl group $(26.3 \mathrm{kcal} / \mathrm{mol})$, as well as for the hydride shift 
that was found to be highest in energy $(25.9 \mathrm{kcal} / \mathrm{mol})$. Shonotl explained this migratory ability of the cyclopropyl group as being a result of delocalization of the positive charge into the three membered ring. Theoretical calculations imply the participation of a pseudo $\pi$-orbital formed from the strained $\mathrm{C}-\mathrm{C}$ bonds with sigma bonds projecting out of the three membered ring skeleton, that stabilizes the transition state with the lowest activation energy of the system, as shown below. 40

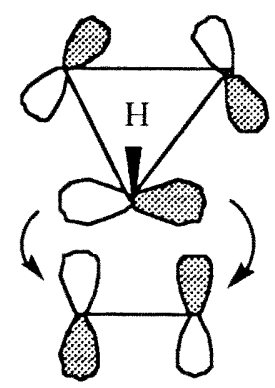

The presence of an ionic intermediate and the involvement of a carbocation that can be stabilized by the presence of a cyclopropyl group are two of the issues involved in this project.

Cramert has reported theoretical studies involving gas phase cycloaddition reactions of ethylene and 1,3-butadiene with the cyclopropylcarbinyl cation. Intensive experimental and theoretical scrutiny using high levels of gas-phase theory and low temperature nuclear magnetic resonance in superacid solvent and amorphous SbF5 agree that the cyclopropylcarbinyl cation can be described as a rapidly equilibrating mixture of cyclopropylmethyl (1) and nonclassical bicyclobutonium (2) cations, Figure IV. 
Although product mixtures obtained from nuclcophilic trapping of the cation typically include homoallylic products, the homoallyl cation (3) lies $30 \mathrm{kcal} / \mathrm{mol}$ higher in energy than (1) and (2), therefore is not a significant contributor to the cation equilibrium population at moderate temperatures as shown in Figure IV.

\section{Figure IV : Cyclopropylcarbinyl Cation Equilibrium}

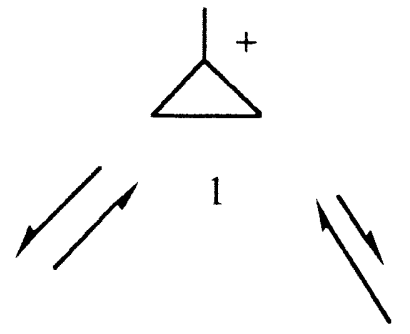

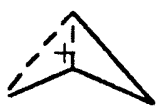

2

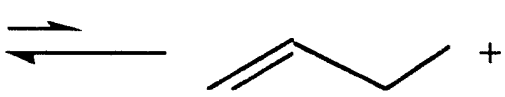

3

Cyclopropylcarbinyl cations are formed when allylic alcohols are treated with $\mathrm{FSO}_{3} \mathrm{H}-\mathrm{SO}_{2} \mathrm{ClF}$ at -78 and $-120^{\circ} \mathrm{C}$. These cations presumably arise through initially-formed allyl cations. Cyclopropylcarbinyl cations (2) are more stable than the allyl cations (1). When the cyclopropylcarbinyl cations (2) are warmed, they rearrange to the more stable allyl cation (3). The cyclopropyl group was estimated to be more stabilizing than the vinyl group by $11-17$ $\mathrm{kcal} / \mathrm{mol} .42$ The relative stabilizing effects of vinyl and cyclopropyl groups are in the order shown below 


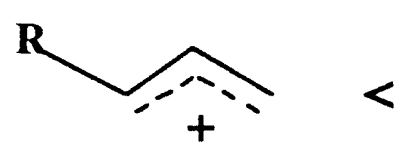

(1)

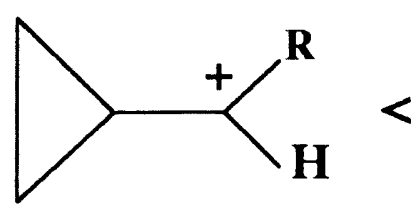

(2)

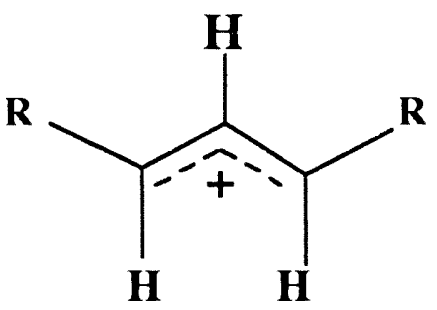

(3)

\section{$\mathbf{R}=$ Alkyl}

Cyclopropane can stabilize electron deficient centers to a much greater extent than cyclobutane or larger carbocycles. ${ }^{+3}$ They interact in the same fashion as an alkene because of enhanced double bond character $\left(\mathrm{sp}^{2}\right)$ due to the small angle in the ring.

The strongly electron rich cyclopropyl ring system is known to undergo facile reaction with a proton. The cyclopropane ring acts as the base (electron donor), and the proton or cationic center acts as the acid (electron acceptor). Another factor controlling the basicity of a hydrocarbon is the energy of its highest occupied molecular orbital (HOMO). The HOMO energy, found by (6-31G), for cyclopropane is $-11.35 \mathrm{eV}$ lower than that of other cycloalkanes.

In addition, the polarizability of cyclopropane is greater than that of other cycloalkanes. This reflects how easily electron density may be shifted in the presence of an electric field, as the one developed by a proton. 32

Frime ${ }^{4+}$ suggests that strain present in either the starting material or the product, plays only a minor role in directing singlet oxygen reactions, but that it has a profound effect on the secondary rearrangements of allylic hydroperoxides formed in the 
photosensitized oxidation of small rings systems. The ground state geometry of the olefin, the interatomic distance between the $\alpha$ olefinic carbon and the $\gamma$-allylic hydrogen (shown below), and the ionization potential of the double bond all have a tremendous effect on the reactivity of olefins with singlet oxygen.

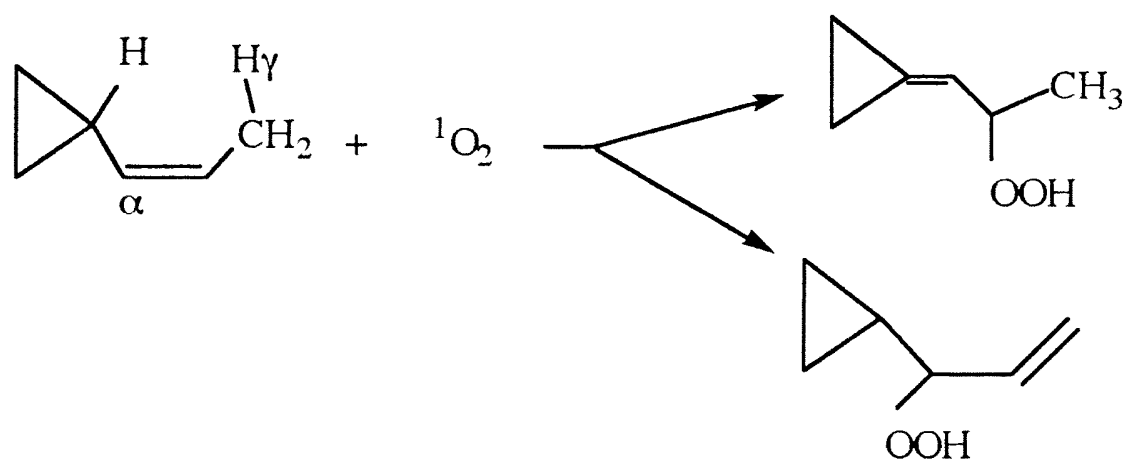

A cyclopropyl group conjugated with a double bond resembles the $\mathrm{sp}^{2}$ characteristics of dienes such as the 2,4-hexadienes. As mentioned above, they also have the advantage that the cyclopropyl group has a greater stabilizing effect than the vinyl group. ${ }^{+2}$

The involvement of the perepoxide in 102 reactions ${ }^{16}$ and evidence for the analogous $A Z I$ in the reactions of RTAD with dienes 15 has been reported. Since dienes and a double bond conjugated with a cyclopropyl group are expected to have similar reactivities. 1,1-Dicyclopropylethylene, cis- and trans-1,2dicyclopropylethylenes were used to probe the reaction pathways of $10_{2}$, RTAD and TCNE. The cyclopropyl groups are expected to stabilize these zwitterionic intermediates such that they maybe trapped with nucleophiles, undergo rearrangement, or be observed directly by low temperature NMR 
The present investigation involving product and solvent trapping studies involving reactions of dicyclopropylethylenes with singlet oxygen and singlet oxygen mimics will help to elucidate the pathway and the possible reaction intermediates present. 
CHAPTER 2

SYNTHESIS AND CHARACTERIZATION OF DICYCLOPROPYLETHYLENES 


\subsection{SYNTHESIS AND CHARACTERIZATION OF 1,1- \\ DICYCLOPROPYLETHYLENE}

1,1-Dicyclopropylethylene was prepared from dicyclopropyl ketone and methyltriphenylphosphonium bromide via Wittig reaction. The yield was $\sim 80 \%$ of pure product, $10 \%$ of unreacted dicyclopropyl ketone, and $10 \%$ unidentified compounds. ${ }^{45}$

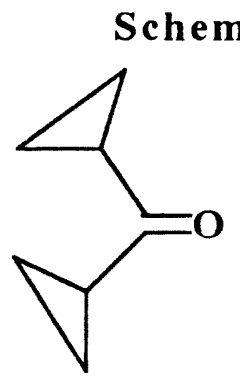

(1)
1) $\mathrm{CH}_{3} \mathrm{P}\left(\mathrm{C}_{6} \mathrm{H}_{5}\right)_{3} \mathrm{Br}$

2) $\mathrm{n}-\mathrm{BuLi} /$ hexane $0^{\circ} \mathrm{C}$ 1,1-dicyclopropylethylene

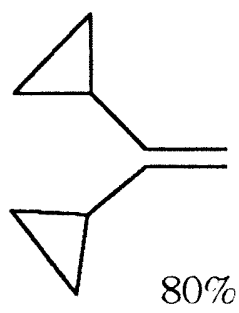

(2)

\section{EXPERIMENTAL:}

Methyltriphenylphosphonium bromide $\left(\begin{array}{llll}11.6 & \mathrm{~g}, & 32.4 & \text { mmoles}\end{array}\right)$ was weighed into a dry, $250 \mathrm{~mL}$ three neck, round bottom flask, and $50 \mathrm{~mL}$ of dry THF (distilled from $\mathrm{Na}$ /benzophenone) was cannulated into the reaction vessel. A positive pressure of argon was maintained throughout the reaction. The suspension was cooled to $0-5{ }^{\circ} \mathrm{C}$, and n-butyllithium, 2.5 $\mathrm{M}$ in hexane $(14.0 \mathrm{~mL}, 32.4$ mmoles $)$ added dropwise from an addition funnel to the well-stirred solution. A yellow color developed upon addition of the base, indicative of formation of the Wittig reagent.

Dicyclopropyl ketone (1) $(3.5 \mathrm{~mL}, 32.4 \mathrm{mmoles})$, NMR spectra (Figures $\mathrm{V} \& \mathrm{VI}$ ), cooled to $0^{\circ} \mathrm{C}$, a bottle with vacuum distilled 
dicyclopropyl ketone was kept in ice-water bath for 30 minutes, then (1) was added dropwise to the Wittig reagent. The color changed from yellow to off-white once the addition was complete. The mixture was allowed to warm slowly to room temperature. The reaction mixture was stirred overnight under a positive pressure of argon.

After $24 \mathrm{hr}$, the reaction mixture was extracted successively with $25 \mathrm{~mL}$ of water and $25 \mathrm{~mL}$ of saturated $\mathrm{NaCl}$ solution. The organic layer was dried over anhydrous $\mathrm{MgSO}_{4}$. The solvent was removed by fractional distillation using a short Vigreux column, and the residue analyzed by gas chromatography (Hewlett Packard 5890, using a DB-5 column, FID detector, $30 \mathrm{~m}$ length, $0.53 \mathrm{~mm}$ id). The reaction mixture consisted of 1,1-dicyclopropylethylene (2) (80\%), unreacted dicyclopropyl ketone (10\%), and unidentified compounds (10\%). Triphenylphosphine oxide (3) was the primary byproduct (Scheme II).

The product was purified by column chromatography on silica gel (60-100 mesh) using hexane as the eluant. The dimensions of the column were 2.5 in long and 2.5 in i.d. The purified 1,1dicyclopropylethylene was characterized by ${ }^{1} \mathrm{H}-$ and $13 \mathrm{C}-\mathrm{NMR}$ (General Electric QE-300 MHz NMR) and GC-Mass spectrometry (Hewlett Packard 5971A, mass selective detector, DB-5 capillary column (MS), $0.25 \mathrm{~mm}, 30 \mathrm{~m}$ length (Figures VII-IX)).

${ }^{1} \mathrm{H}-\mathrm{NMR}\left(300 \mathrm{MHz}, \mathrm{CDCl}_{3}\right) \delta(\mathrm{ppm}): 4.58 \quad(\mathrm{~s}, 2 \mathrm{H}), 1.28-1.30 \quad(\mathrm{~m}$, $2 \mathrm{H}), \quad 0.53-0.54(\mathrm{~m}, 4 \mathrm{H}), 0.45-0.46(\mathrm{~m}, 4 \mathrm{H}) ; 1{ }^{3} \mathrm{C} \quad \mathrm{NMR}(\mathrm{ppm}): 153.2 \quad(\mathrm{~s})$, 105.6 (t), 16.4 (d), 6.9 (t); MS m/z $108(\mathrm{M}), 77(\mathrm{M}-31), 65(\mathrm{M}-43)$. 
Figure V: 1 H NMR SPECTRUM OF DICYCLOPROPYL KETONE

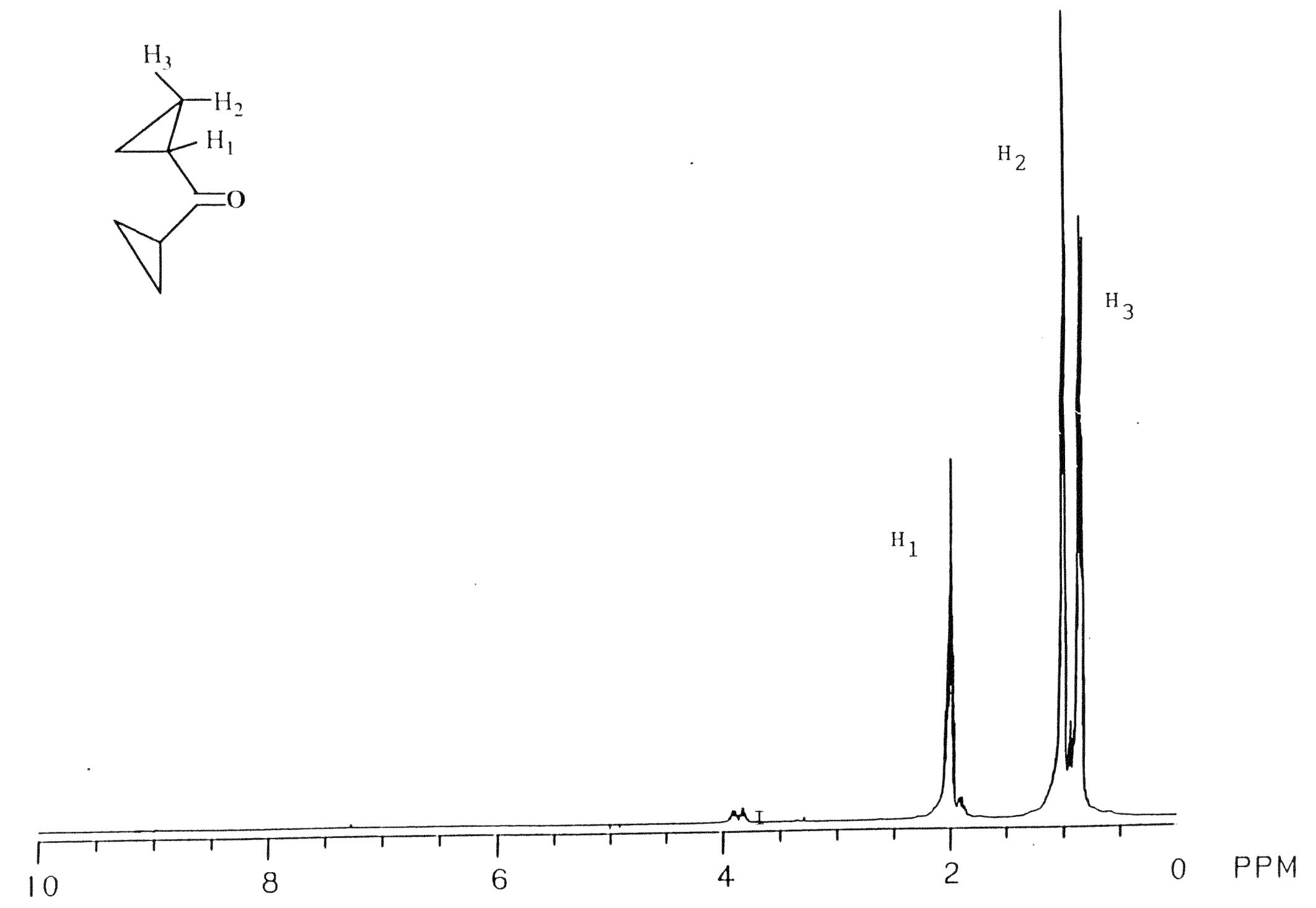


Figure VI: $13^{\mathrm{C}}$ NMR SPECTRUM OF DICYCLOPROPYL KETONE

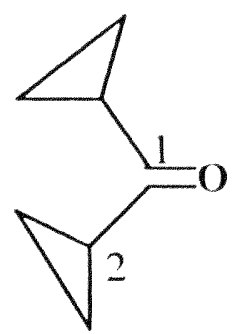

3

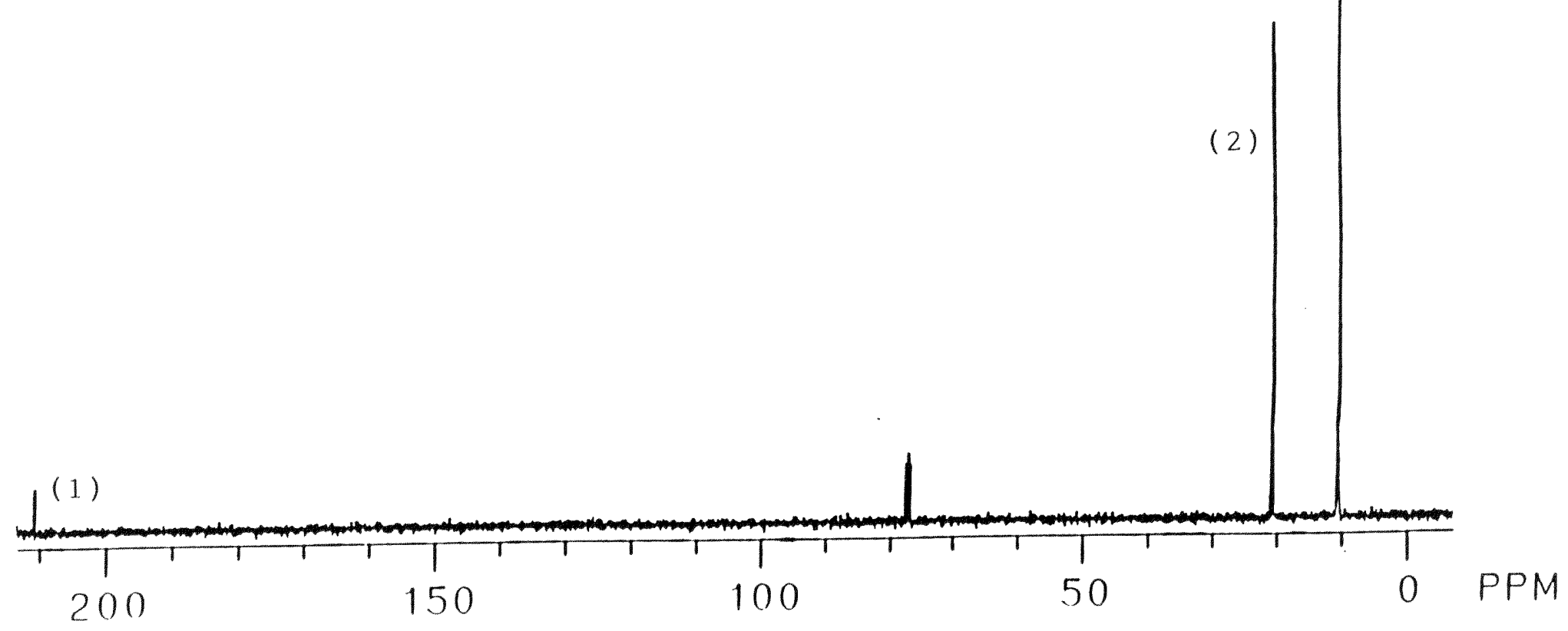


Figure VII: 1H NMR SPECTRUM OF 1,1-DICYCLOPROPYLETHYLENE

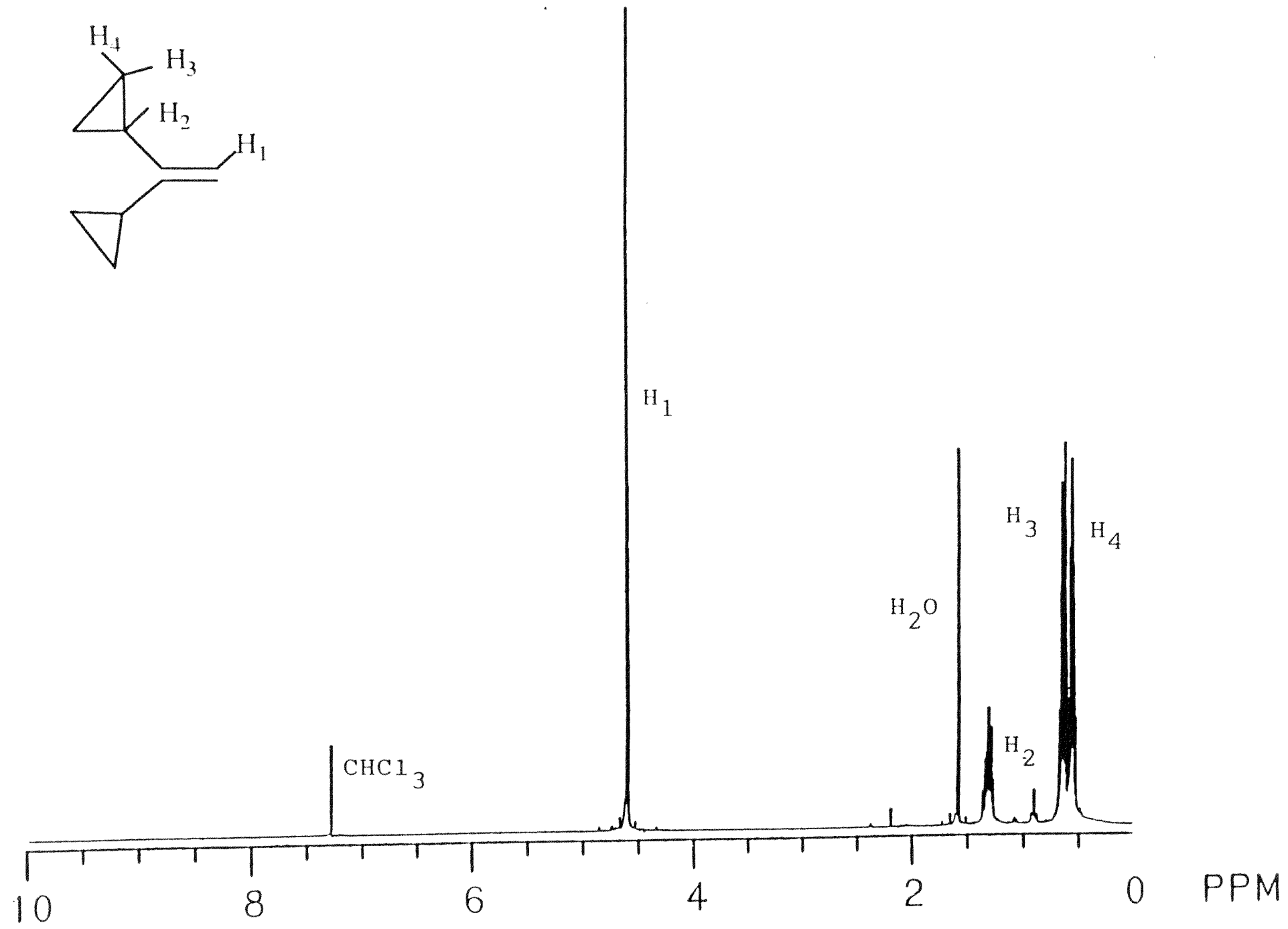


Figure VIII: $13^{3}$ C NMR SPECTRUM OF 1,1-DICYCLOPROPYLETHYLENE
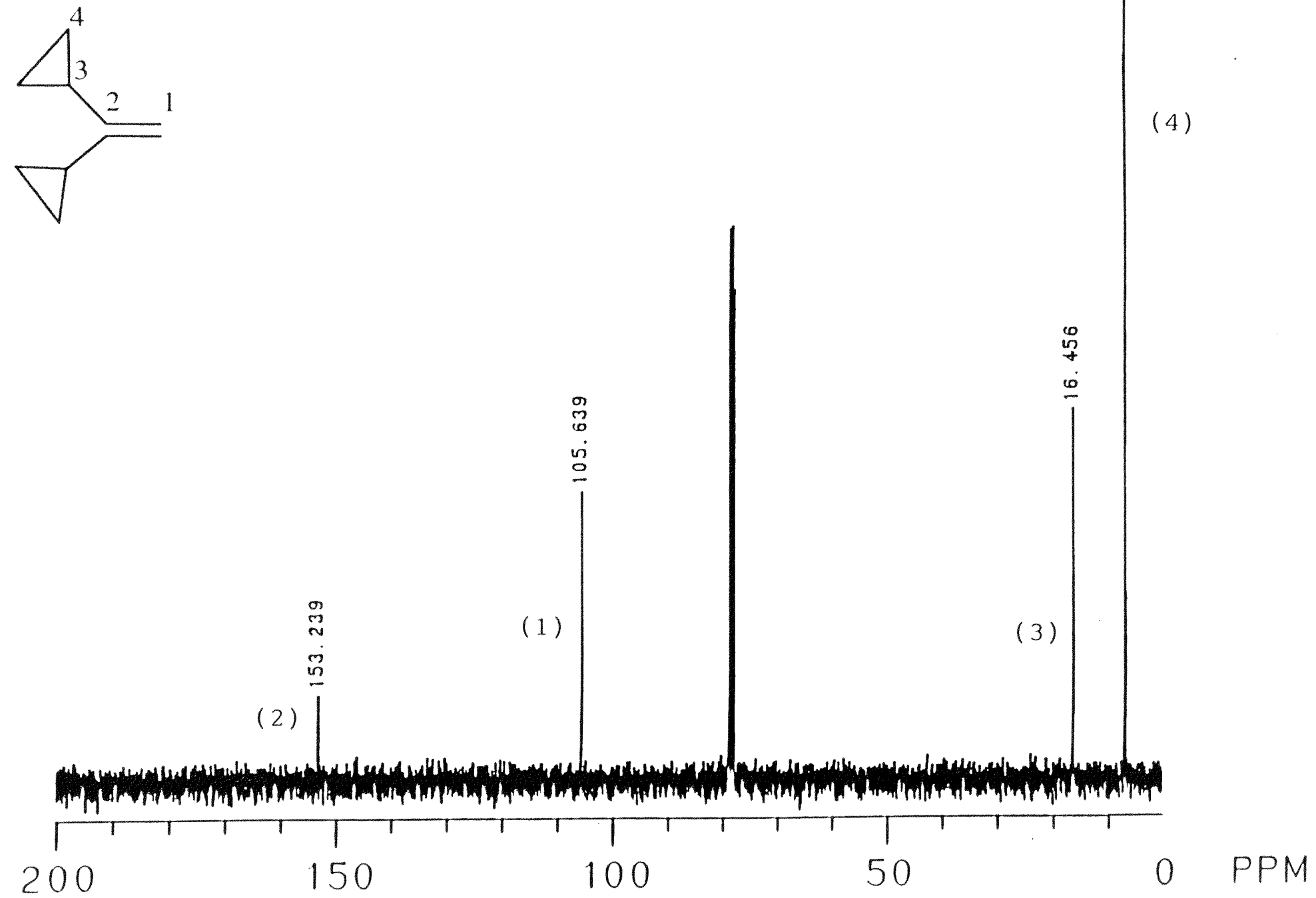
Figure IX: MASS SPECTRUM OF 1,1-DICYCLOPROPYLETHYLENE

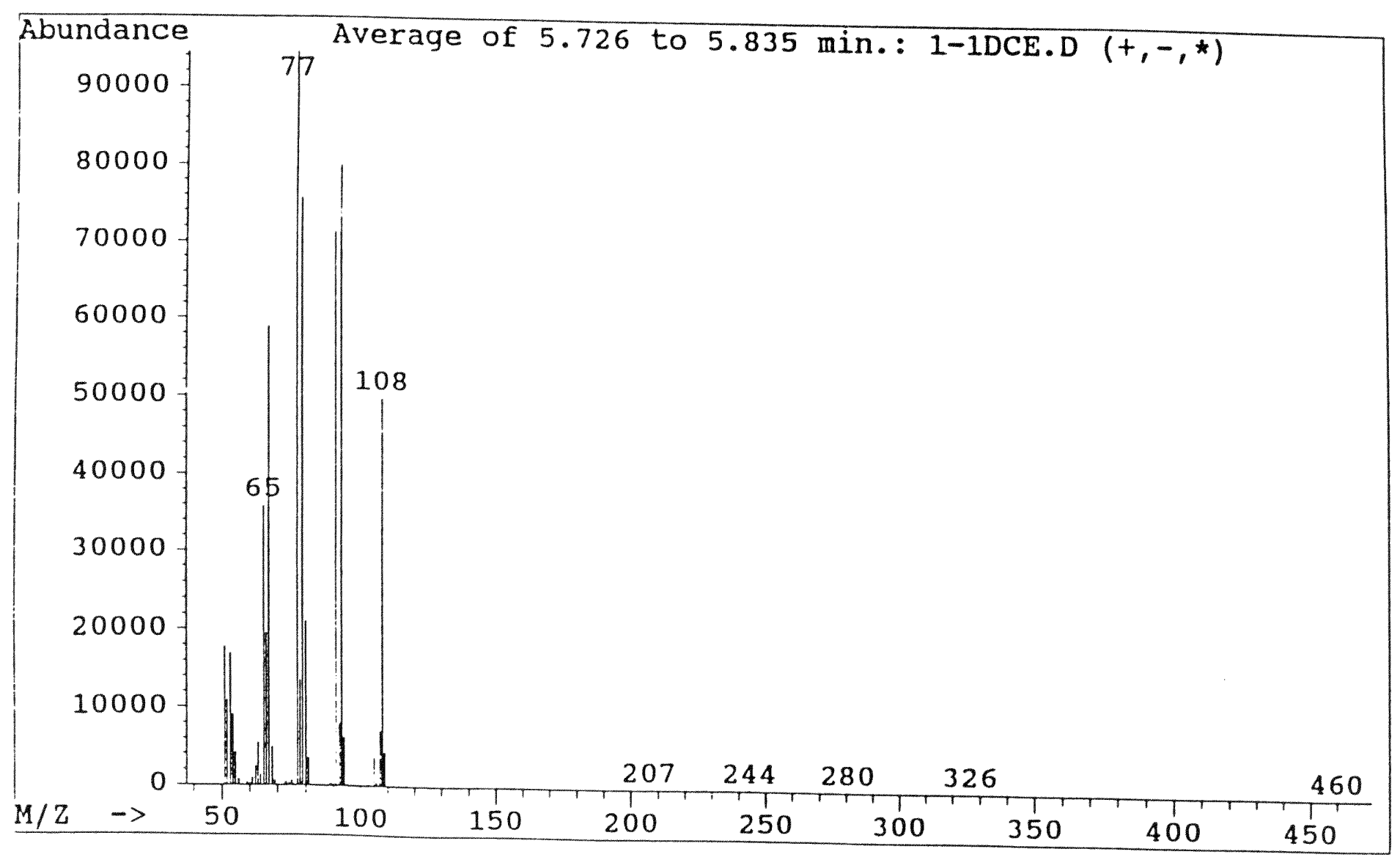




\subsection{SYNTHESIS AND CHARACTERIZATION OF $c$ is -1, 2- DICYCLOPROPYLETHYLENE}

Typical Wittig reaction methodology was employed using cyclopropylmethyltriphenylphosphonium bromide, cyclopropanecarboxaldehyde and phenyllithium which were heated to reflux in benzene for $24 \mathrm{hr}$. A mixture of cis- and transdicyclopropylethylenes was obtained following the procedure of Maercker.6 The resulting mixture was composed of $60 \%$ cis- and 40\% trans-dicyclopropylethylene as determined by gas chromatography. Attempts to separate the transdicyclopropylethylene from the mixture by selective liquid-liquid extraction with $\mathrm{AgNO}_{3}$ aqueous solution ${ }^{7}$ were unsuccessful.

A second trial was performed using the Fritsch-ButtenbergWiechell rearrangement. +8 This three-step synthesis was accomplished by the reaction of dicyclopropyl ketone with (chloromethyl)triphenylphosphonium chloride affording 1-chloro2,2-dicyclopropylethylene as the initial product.

Treatment of 1-chloro-2,2-dicyclopropylethylene with nbutyllithium in hexane at room temperature afforded 1,2dicyclopropylethyne as the rearrangement product. ${ }^{49}$ Hydrogenation under a maximum pressure of $10 \mathrm{psi}$ of $\mathrm{H}_{2}$ at room temperature for 90 minutes using a Parr hydrogenator which yielded cisdicyclopropylethylene in a nearly quantitative yield. 


\subsubsection{SYNTHESIS OF $c$ is-DICYCLOPROPYLETHYLENE VIA WITTIG REACTION}

\section{EXPERIMENTAL:}

Triphenylphosphine (3.9 g, $14.8 \mathrm{mmoles})$ was placed into a 300 $\mathrm{mL}$ round bottom flask, equipped with a condenser which was cooled with tap water. Dry benzene $(100 \mathrm{~mL})$ (distilled over sodium/benzophenone) was cannulated into the reaction vessel, and a positive pressure of argon and magnetic stirring were maintained throughout the reaction sequence.

Cyclopropylmethyl bromide $(2.0 \mathrm{~g}, 14.8 \mathrm{mmoles})$ was added to the solution via syringe. The reaction mixture was heated at reflux for 10 days producing insoluble cyclopropylmethyltriphenylphosphonium bromide (Scheme III).

Scheme III: Synthesis of Phosphonium Salt

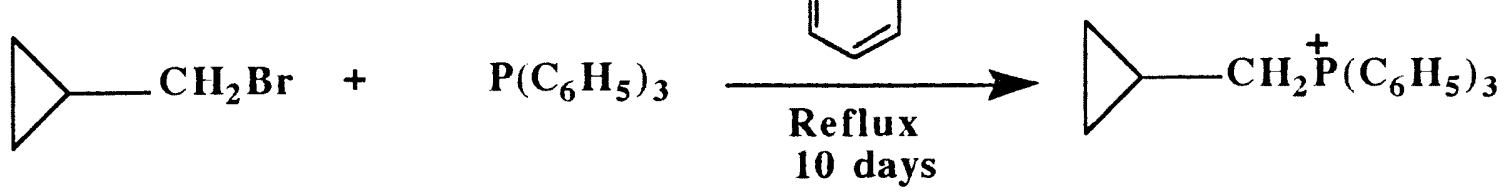

Cyclopropylmethyltriphenylphosphonium bromide (14.8 mmoles) in benzene was allowed to cool at room temperature, and phenyllithium $1.8 \mathrm{M}$ in cyclohexane/ether $(9.6 \mathrm{~mL}, 14.8$ mmoles $)$ was added dropwise using an addition funnel, under an argon 
atmosphere. The addition was accompanied by a color change from yellow to dark red (Scheme IV).

Scheme IV: Preparation of Wittig Reagent

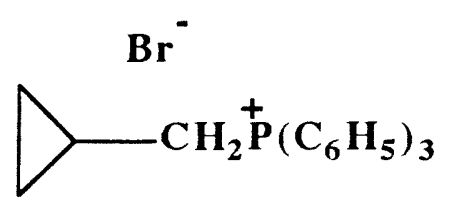

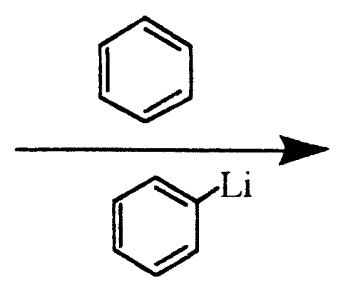

$20{ }^{\circ} \mathrm{C}$

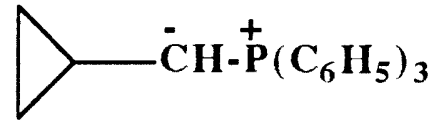

After 45 min of stirring, cyclopropanecarboxaldehyde $(1.0 \mathrm{~g}$, 14.8 mmoles) in $-5.0 \mathrm{~mL}$ of dry THF was added slowly through the addition funnel to the well-stirred suspension. The mixture turned orange during the addition and brown after heating at reflux for 24 hours (Scheme V).

Scheme V: Alkene Synthesis via Wittig Reaction
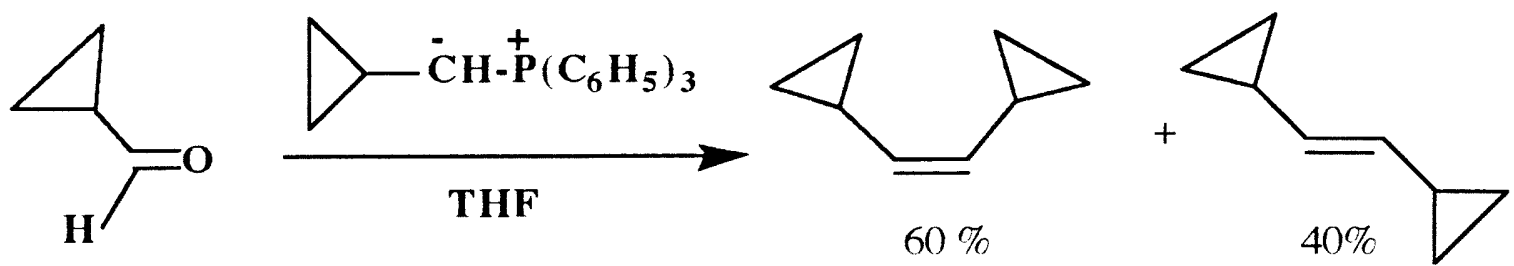

The work-up procedure consisted of washing the reaction mixture with water and then filtering off the insoluble lithium bromide. Triphenylphosphine oxide is very soluble in benzene and remained in the organic phase. The majority of solvent was removed by fractional distillation. 
Several methods were used in an attempt to remove the high and low boiling impurities: First, liquid chromatography on silica gel (60-100 mesh), (3 in length and 1.0 in i.d.) using pentane as the eluant. The results were moderately successful; the majority of the triphenylphosphine oxide was removed.

The 1,2-dicyclopropylethylenes were trapped under vacuum using an acetone-dry ice trap. This procedure led to substantial loss of product, but it was effective in removing the benzene from the mixture. Upon removal of the benzene, the remaining triphenylphosphine oxide precipitated from the solution and was separated by gravity filtration.

The cis-and trans-dicyclopropylethylenes were identified by gas chromatography (Hewlett Packard 5890 using a DB-17 column (J \& W Scientific), $30 \mathrm{~m}$ length, $0.25 \mathrm{~mm}$ id and pressure-temperature program. The yield were $60 \%$ cis- and $40 \%$ transdicyclopropylethylene.

Separation of the cis-and trans-isomers was attempted by extracting a hexane solution of the isomers with aqueous $20 \% \mathrm{AgNO}_{3}$ solution. ${ }^{47}$ The technique was adapted to the dicyclopropylethylene isomers as follows: A liquid-liquid extraction was performed using $20 \mathrm{~mL}$ of $20 \% \mathrm{AgNO}_{3}$ aqueous solution and $10 \mathrm{~mL}$ of 1,2dicyclopropylethylenes/hexane solution. The mixture was placed in a $30 \mathrm{~mL}$ vial and shaken for $90 \mathrm{~min}$ in an orbital shaker. The organic phase was separated, and the hexane removed by fractional distillation. The aqueous phase was treated with concentrated $\mathrm{NH}_{4} \mathrm{OH}$ containing crushed ice in an attempt to break the hydrocarbon-olefin 
silver complex. The resulting solution was extracted with an equal amount of pentane. The organic phase was dried over $\cdot \mathrm{MgSO}_{4}$ and filtered, and the reaction mixture analyzed by gas chromatography.

GC analysis showed that the separation was not successful. Cyclopropylmethyl bromide was recovered in significant amounts following the extraction process. This impurity may have an affinity for complexation with $\mathrm{AgNO}_{3}$ thus preventing selective complexation and separation of the cis - and trans-isomers.

\subsubsection{SYNTHESIS OF $c$ is-DICYCLOPROPYLETHYLENE BY FRITSCH-BUTTENBERG-WIECHELL REARRANGEMENT}

\section{EXPERIMENTAL:}

To a $100 \mathrm{~mL}$ three-neck round bottom flask purged with argon were added (chloromethyl)triphenylphosphonium chloride $(6.1 \mathrm{~g}$, 17.7 mmoles), dry THF (30 $\mathrm{mL})$ and pyridine (1.6 g, 17.7 mmoles). The reaction mixture was stirred magnetically for two hours at room temperature under a positive pressure of argon. Then n-butyllithium $(8.0 \mathrm{~mL}$ of a $2.5 \mathrm{M}$ solution in hexane, $20 \mathrm{mmoles})$ was added dropwise. The suspension developed an orange color which persisted and darkencd. The reaction mixture was maintained at $20{ }^{\circ} \mathrm{C}$, and dicyclopropyl ketone (1), purified by vacuum distillation, $(2.0 \mathrm{~g}, 17.7$ mmoles) in $5 \mathrm{~mL}$ of dry THF was added dropwise to the mixture.

The resulting solution was treated with $25 \mathrm{~mL}$ of $10 \% \quad \mathrm{H}_{2} \mathrm{SO}_{4}$ and extracted with two $50 \mathrm{~mL}$ portions of ether. The organic layer 
was washed successively with water and saturated $\mathrm{NaCl}$ solution and dried for 5 min over anhydrous $\mathrm{MgSO}_{4}$. The $\mathrm{MgSO}_{4}$ was removed by gravity filtration.

The solvent was removed at a reduced pressure, and the residue was placed in the freezer to initiate crystallization of triphenylphosphine oxide. After 24 hours, substantial crystallization had occurred, and the supernatant liquid was decanted from the crystals. The resulting solution was analyzed by gas chromatography (Hewlett Packard 5890, using a DB-5 column, FID detector, $30 \mathrm{~m}$ length, $0.53 \mathrm{~mm}$ i.d.). It consisted of 1-chloro-2,2-dicyclopropylethylene (4) $(85 \%)$, unreacted dicyclopropyl ketone (10\%), and triphenylphosphine oxide (5\%), (Scheme VI).

Scheme VI: Synthesis of 1-chloro-2,2-dicyclopropylethylene

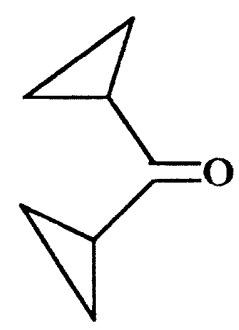

( 1$)$

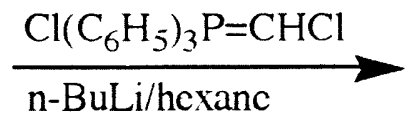

R. T.

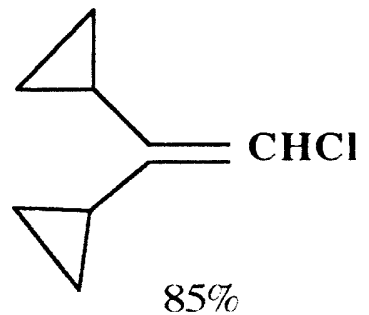

(4)

The desired product, 1-chloro-2,2-dicyclopropylethylene, was chromatographed on silica gel (60-100 mesh), (3.0 in length and 2.5 in i.d.), using hexane as the eluant. The purified 1-chloro-2,2dicyclopropylethylene was characterized by $1_{\mathrm{H}-}, 13^{3} \mathrm{C}-\mathrm{NMR}$ and GCMass Spectrometry (Figures X-XII). 
${ }^{1} \mathrm{H}-\mathrm{NMR}\left(300 \mathrm{MHz}, \mathrm{CDCl}_{3}\right) \quad(\mathrm{ppm}): \delta 5.7(\mathrm{~s}, 1 \mathrm{H}), 1.96-2.01 \quad(\mathrm{~m}$, $1 \mathrm{H}), \quad 0.81-0.86(\mathrm{~m}, 1 \mathrm{H}), 0.76(\mathrm{dd}, 2 \mathrm{H}), \quad 0.64-0.70(\mathrm{~m}, 2 \mathrm{H}), \quad 0.50(\mathrm{~m}$, $2 \mathrm{H}), 0.28(\mathrm{~m}, 2 \mathrm{H}) ; 13 \mathrm{C}-\mathrm{NMR}\left(300 \mathrm{MHz}, \mathrm{CDCl}_{3}\right): \delta 144.0$ (s), 114.0 (d), 14.3 (d), 12.5 (d), 6.2 (t), 5.5 (t); MS m/z $142\left(\mathrm{M}^{+}\right), 105\left(\mathrm{M}^{+} .-37\right), 91$

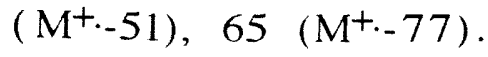


Figure X: 1 H NMR SPECTRUM OF 1-CHLORO-2,2-DICYCLOPROPYLETHYLENE
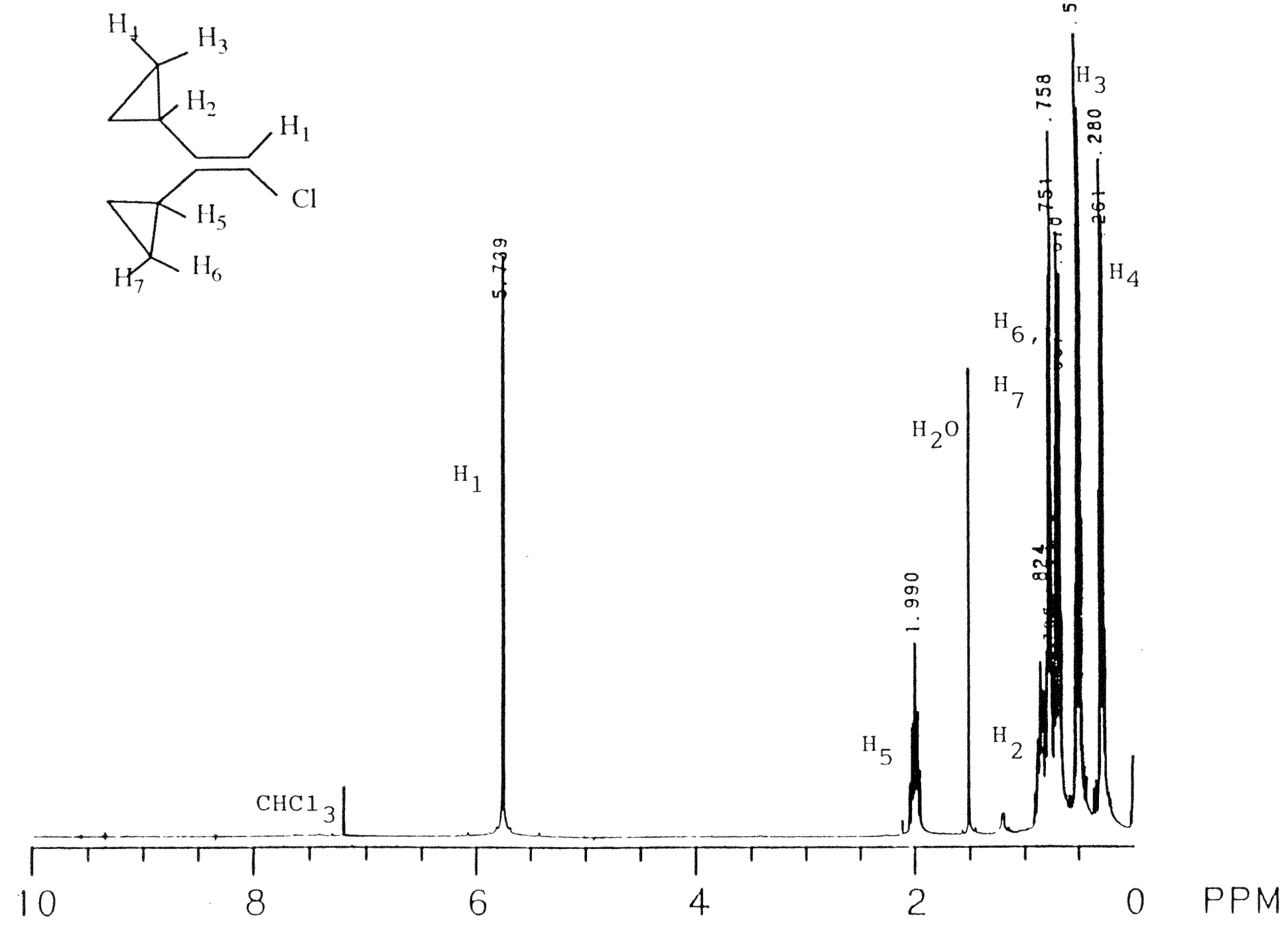
Figure XI: 1 3C NMR SPECTRUM OF 1-CHLORO-2,2-DICYCLOPROPYLETHYLENE
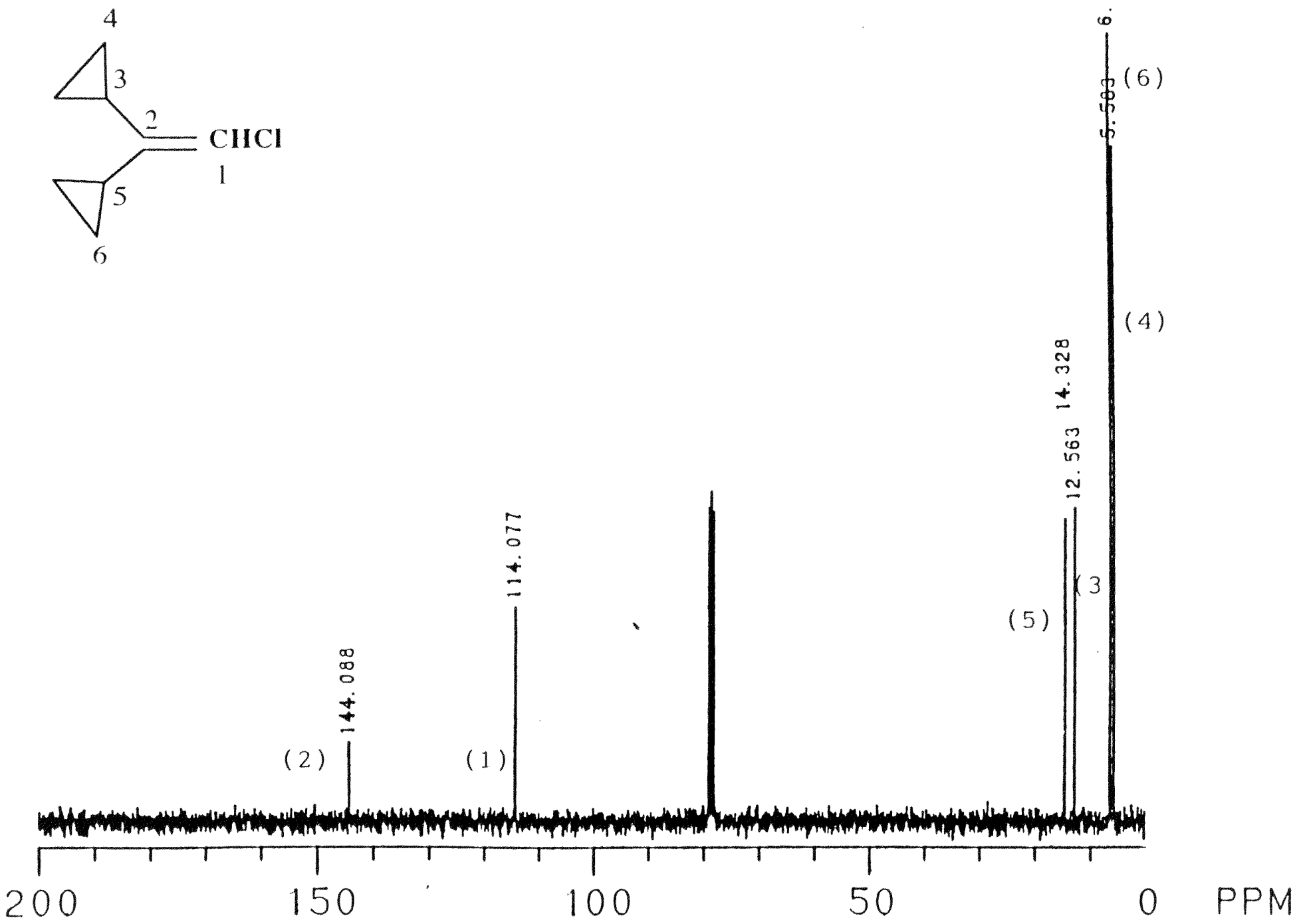
Figure XII: MASS SPECTRUM OF 1-CHLORO-2,2-DICYCLOPROPYLETHYLENE

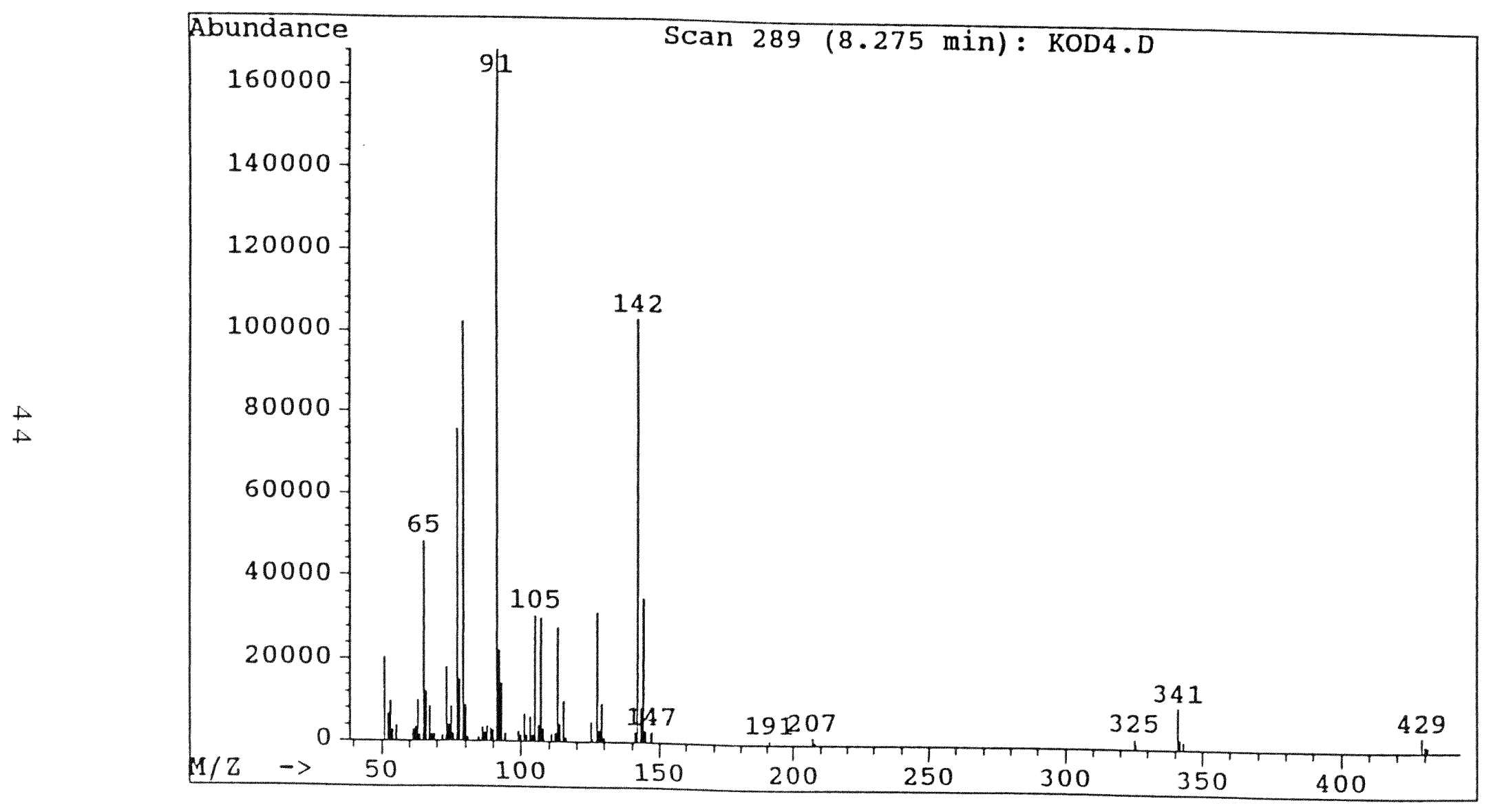


(Step II): 1-Chloro-2,2-dicyclopropylethylene (1.6 g, 11.1 mmoles) and dry THF (15 mL) were placed in a $50 \mathrm{~mL}$ three-neck round bottom flask, and n-butyllithium $(5.4 \mathrm{~mL}$ of $2.5 \mathrm{M}$ solution in hexane, 11.1 mmoles) added dropwise with the formation of a brown color. The solution was stirred overnight at room temperature under an argon atmospherere. The resulting solution was mixed with $25 \mathrm{~mL}$ of diethyl ether. The organic phase was extracted successively with water and saturated $\mathrm{NaCl}$ solution and then dried over anhydrous $\mathrm{MgSO}_{4}$. After filtering off the $\mathrm{MgSO}_{4}$, the ether was removed by fractional distillation. 1,2-Dicyclopropylethyne (5) (Scheme VII) was separated from the biproduct, 1,1-dicyclopropyl-1-hexene, and unreacted starting material, 1-chloro-2,2-dicyclopropylethylene, by column chromatography on silica gel (60-100 mesh) (3.0 in length, 2.5 in i.d.) using pentane as the eluant. The product was obtained in $90 \%$ yield and was characterized by $1_{\mathrm{H}-}, 13 \mathrm{C}-\mathrm{NMR}$ and GC-Mass Spectrometry (Figures XIII-XV).

${ }^{1} \mathrm{H}-\mathrm{NMR}(300 \mathrm{MHz}, \mathrm{CDCl} 3) \delta(\mathrm{ppm}): 1.07-1.12(\mathrm{~m}, 2 \mathrm{H}), \quad 0.61-$ $0.64(\mathrm{~m}, 4 \mathrm{H}), 0.49-0.52(\mathrm{~m}, 4 \mathrm{H}) ; 1{ }^{3} \mathrm{C}-\mathrm{NMR}: 79.2(\mathrm{~s}), 8.51(\mathrm{t}), 0.0(\mathrm{~d})$; MS m/z $106 \quad\left(\mathrm{M}^{+}\right), 91 \quad(\mathrm{M}-15), 77$ (M-29), 51 (M-55).

Scheme VII: Dehydrochlorination of 1-chloro-2,2dicyclopropylet hylene.

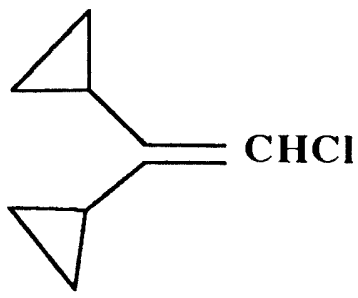

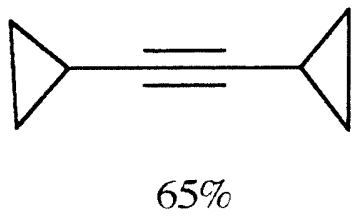

(5) 
Figure XIII: ${ }^{1}$ H NMR SPECTRUM OF 1,2-DICYCLOPROPYLETHYNE

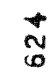
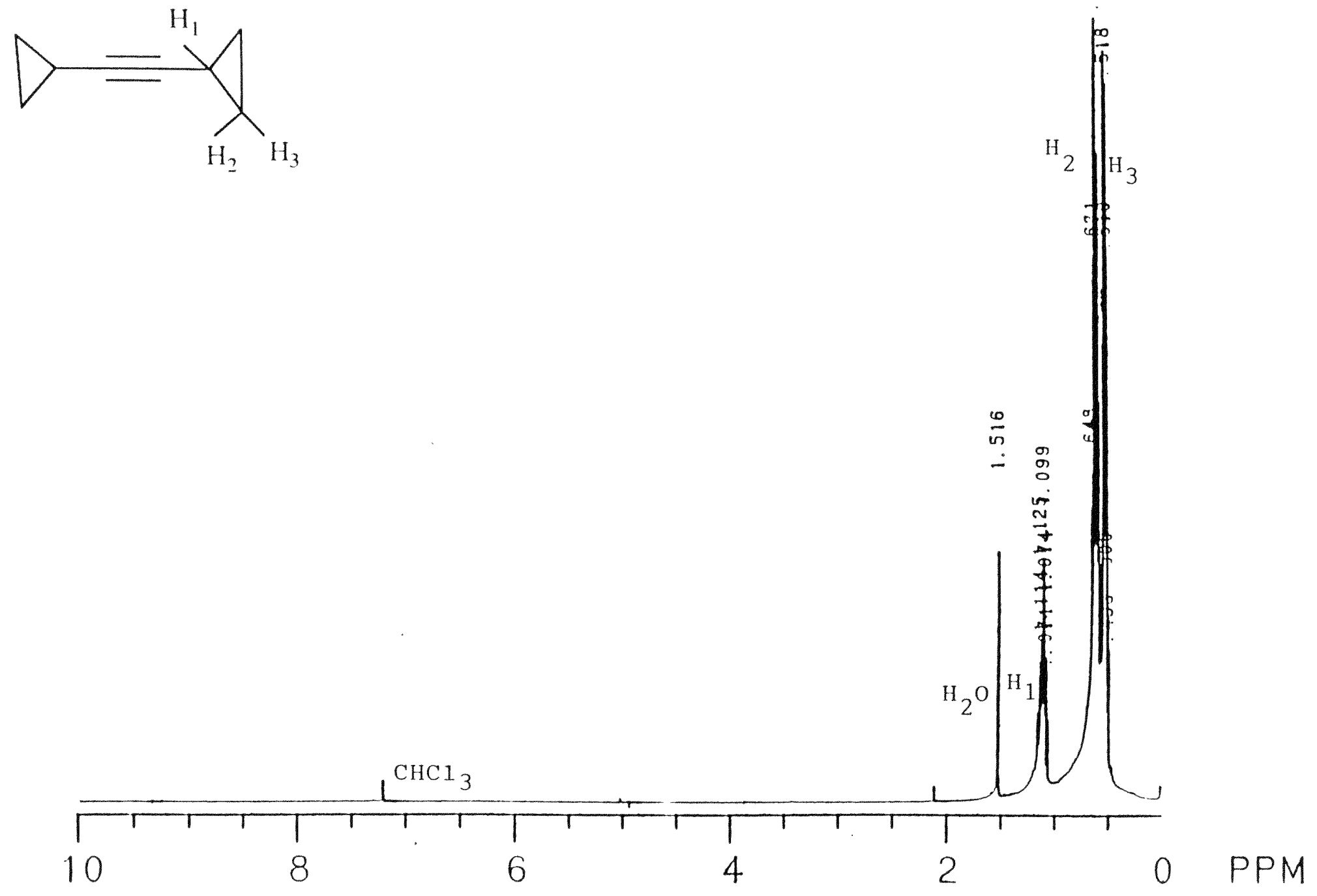
Figure XIV: $13^{3}$ N NMR SPECTRUM OF 1,2-DICYCLOPROPYLETHYNE
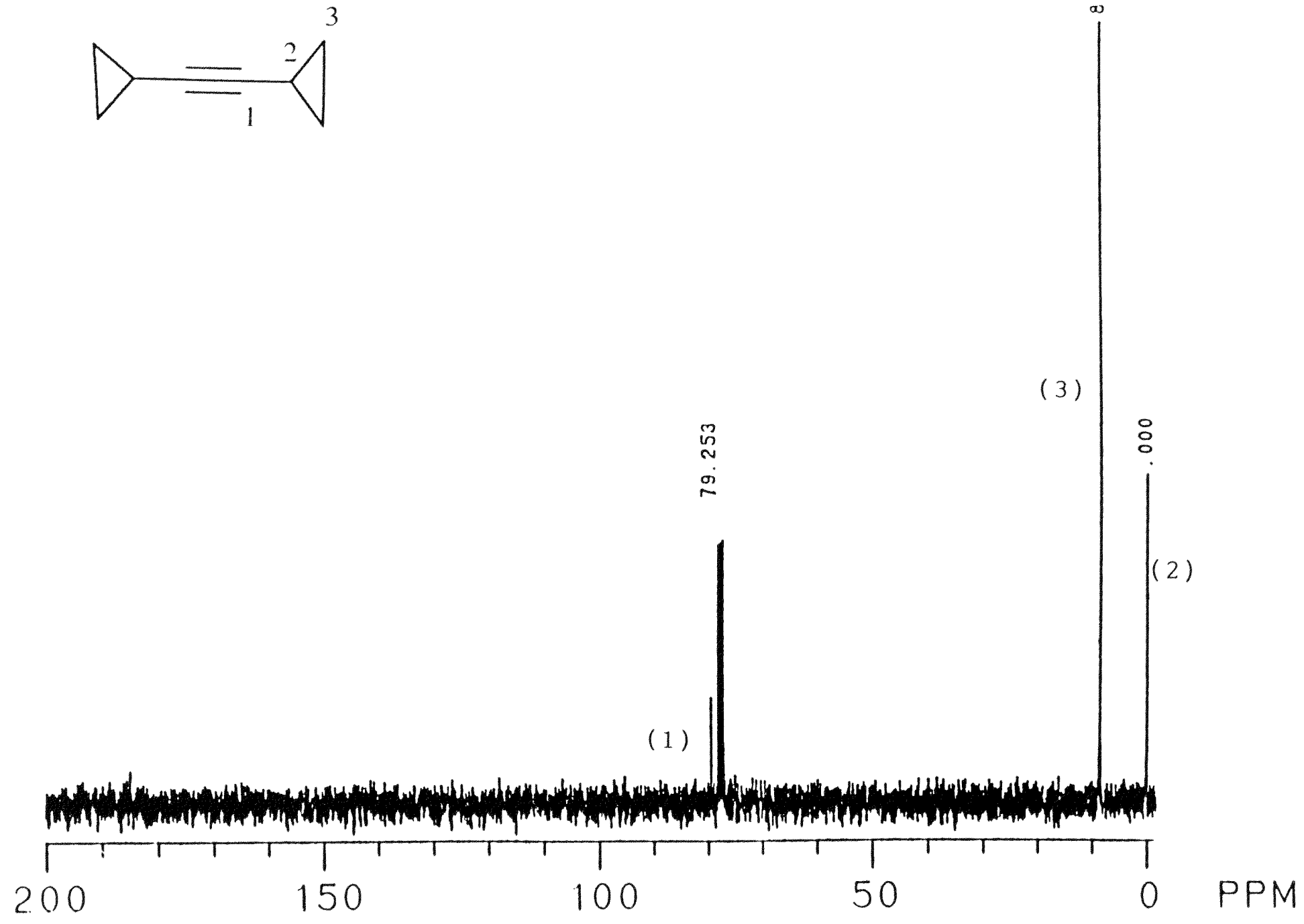
Figure XV: MASS SPECTRUM OF 1,2-DICYCLOPROPYLETHYNE

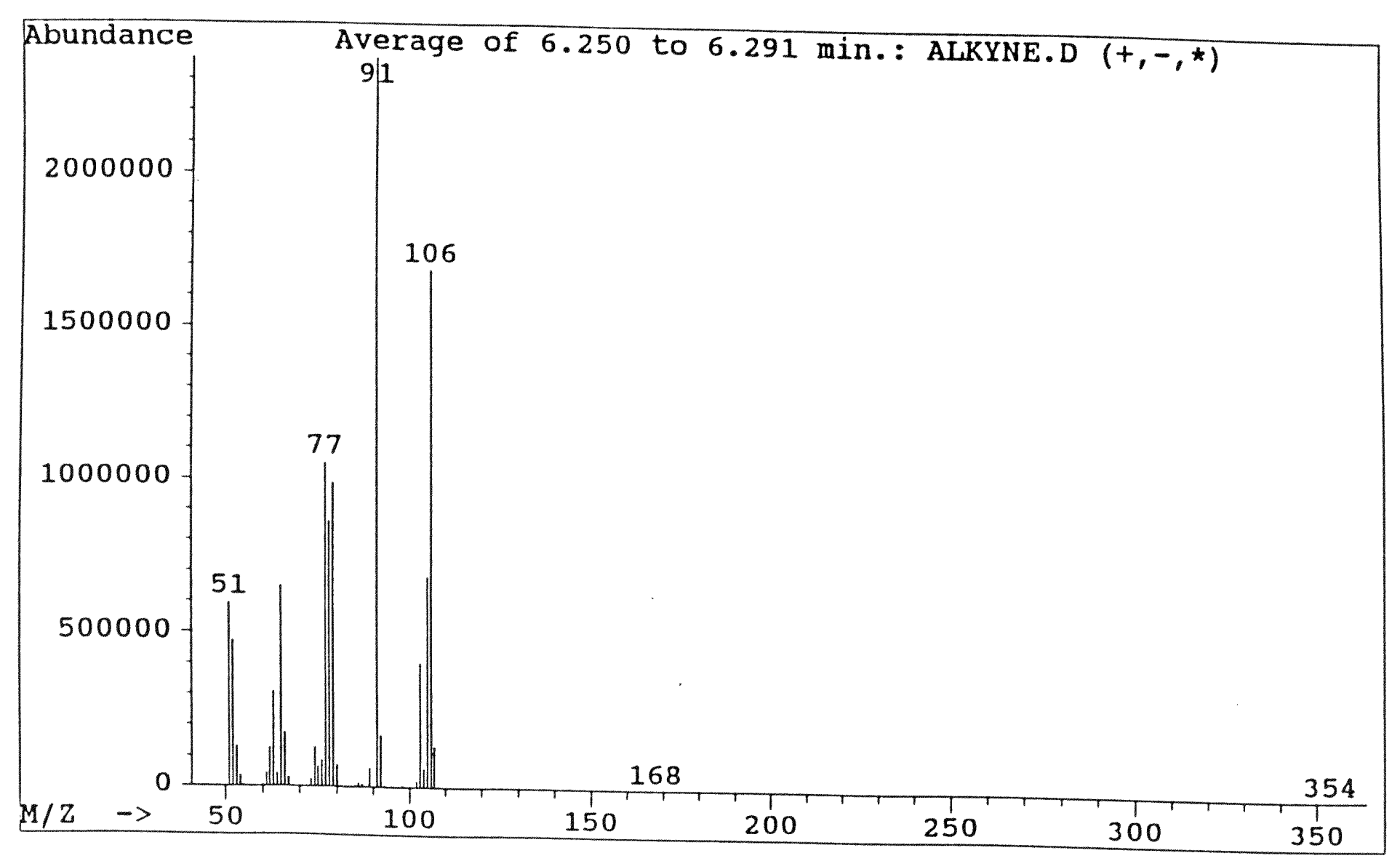


Hydrogenation of 1,2-Dicyclopropylethyne (Step III): 1,2Dicyclopropylethyne $(0.5 \mathrm{~g}, 4.7$ mmoles $)$ was mixed with $100 \mathrm{~mL}$ reagent-grade pentane (previously dried for 5 min with $\mathrm{MgSO}_{4}$ ), Lindlar's catalyst $(0.2 \mathrm{~g})$, and quinoline $(0.1 \mathrm{~g}, 4.7$ mmoles $)$ in a 500 $\mathrm{mL}$ Parr bottle. Hydrogenation was carricd out in a Parr hydrogenation apparatus under 10 psi of hydrogen for $90 \mathrm{~min}$ at room temperature.

The cis- and trans-1,2-dicyclopropylethylenes were obtained in $-90 \%$ and $-10 \%$ yield respectively (Scheme VIII) as determined by gas chromatography. The products were further purified from highboiling impurities by preparative gas chromatography using a GOWMAC, series 350, gas chromatograph (DC 200 column, injector $160{ }^{\circ} \mathrm{C}$, detector $170{ }^{\circ} \mathrm{C}$, column $150^{\circ} \mathrm{C}$ ). The cis-1,2-dicyclopropylethylene (6) was obtained $90 \%$ pure. Final characterization was determined by ${ }^{1} \mathrm{H}-,{ }^{1}{ }^{3} \mathrm{C}-\mathrm{NMR}$ and GC-MS (Figures XVI-XVIII).

${ }^{1} \mathrm{H}-\mathrm{NMR}\left(300 \mathrm{MHz}, \mathrm{CDCl}_{3}\right) \delta_{-}(\mathrm{ppm}): 4.70-4.72(\mathrm{dd}, 2 \mathrm{H}), 1.69-$ $1.72(\mathrm{~m}, \quad 2 \mathrm{H}), \quad 0.76-0.78 \quad(\mathrm{dd}, \quad 4 \mathrm{H}), \quad 0.32-0.35 \quad(\mathrm{dd}, \quad 4 \mathrm{H}) ; \quad 13 \mathrm{C}-\mathrm{NMR}$ $\left(\mathrm{CDCl}_{3}\right): 132.2$ (d), 9.9 (d), 6.9 (t); $\mathrm{MS} \mathrm{m} / \mathrm{z} 108 \quad\left(\mathrm{M}^{+}\right), 91 \quad(\mathrm{M}-17), 77$ $(M-31), 67(M-41)$.

Scheme VIII: Hydrogenation of 1,2-dicyclopropylethyne

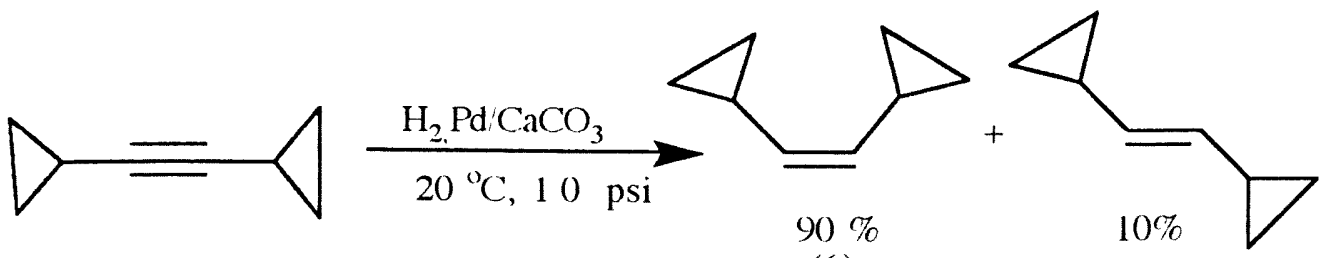

(6) 
Figure XVI: 1 H NMR SPECTRUM OF CIS-DICYCLOPROPYLETHYLENE
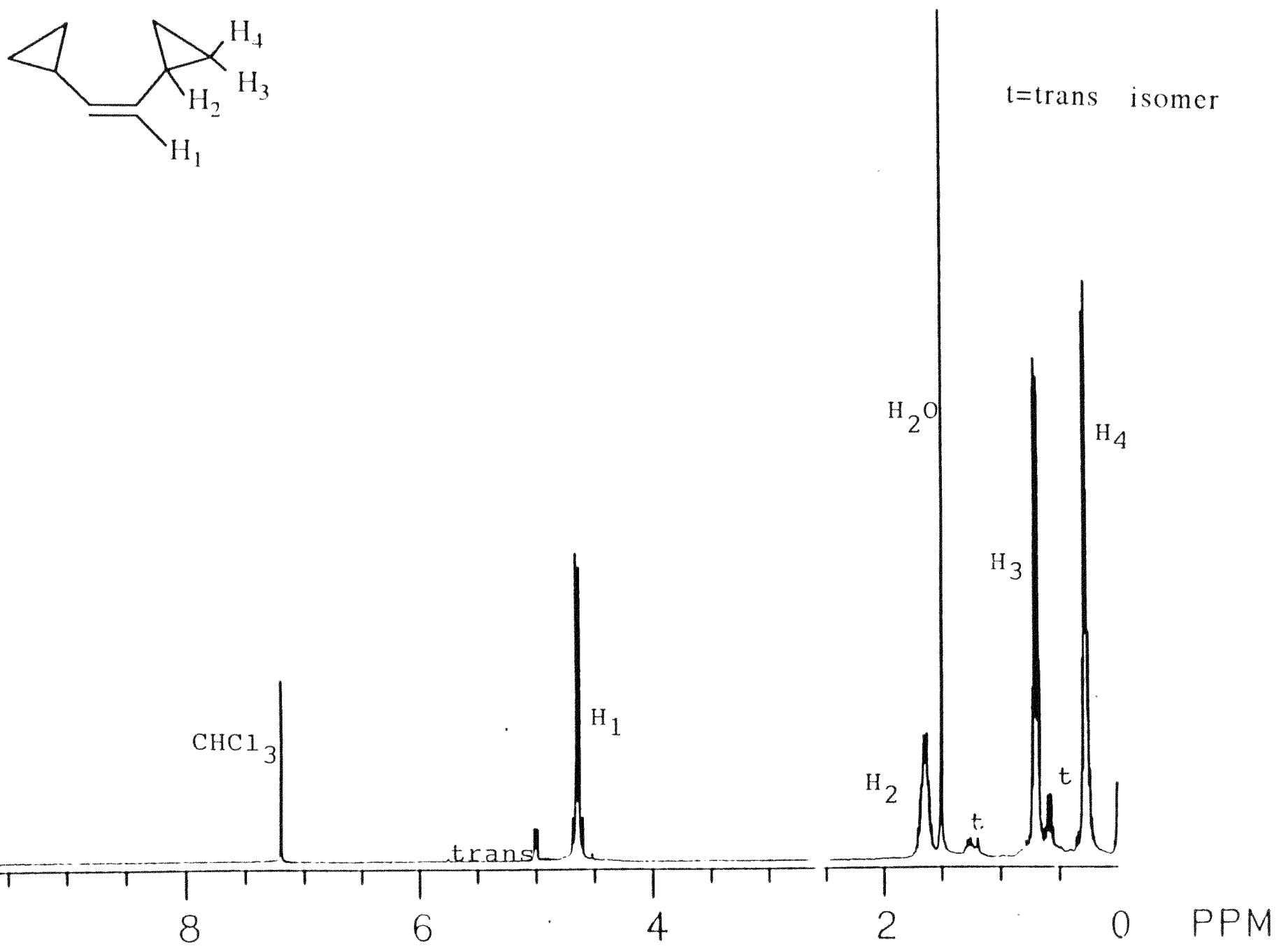
Figure XVII: 1 3 C NMR SPECTRUM OF CIS-DICYCLOPROPYLETHYLENE

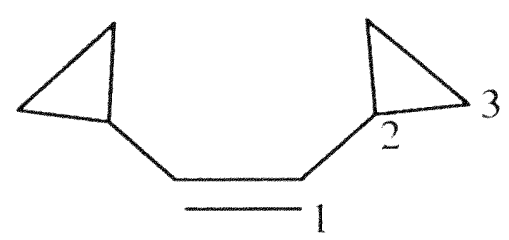

$$
t=\text { trans isomer }
$$

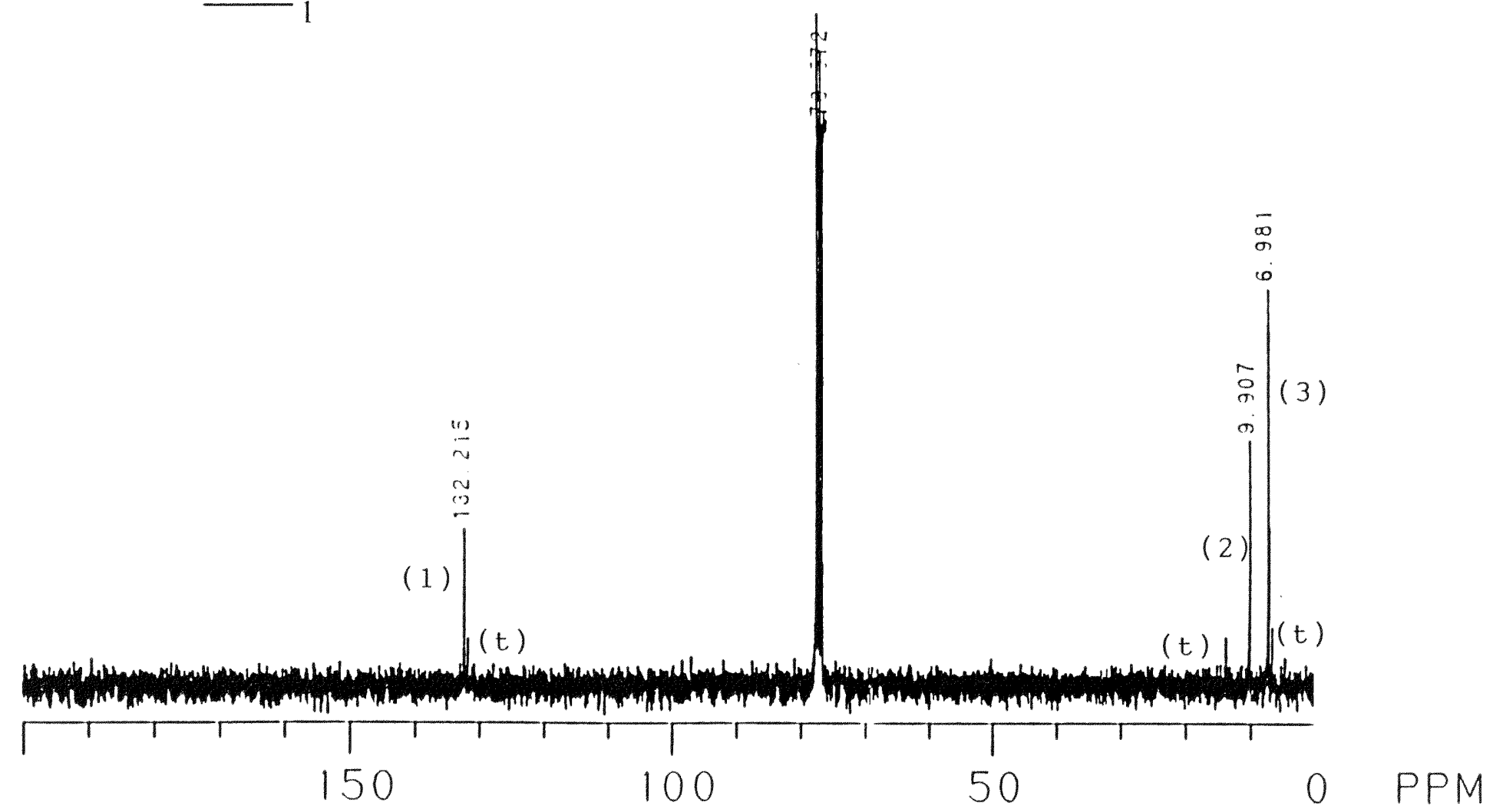


Figure XVIII: MASS SPECTRUM OF CIS-DICYCLOPROPYLETHYLENE

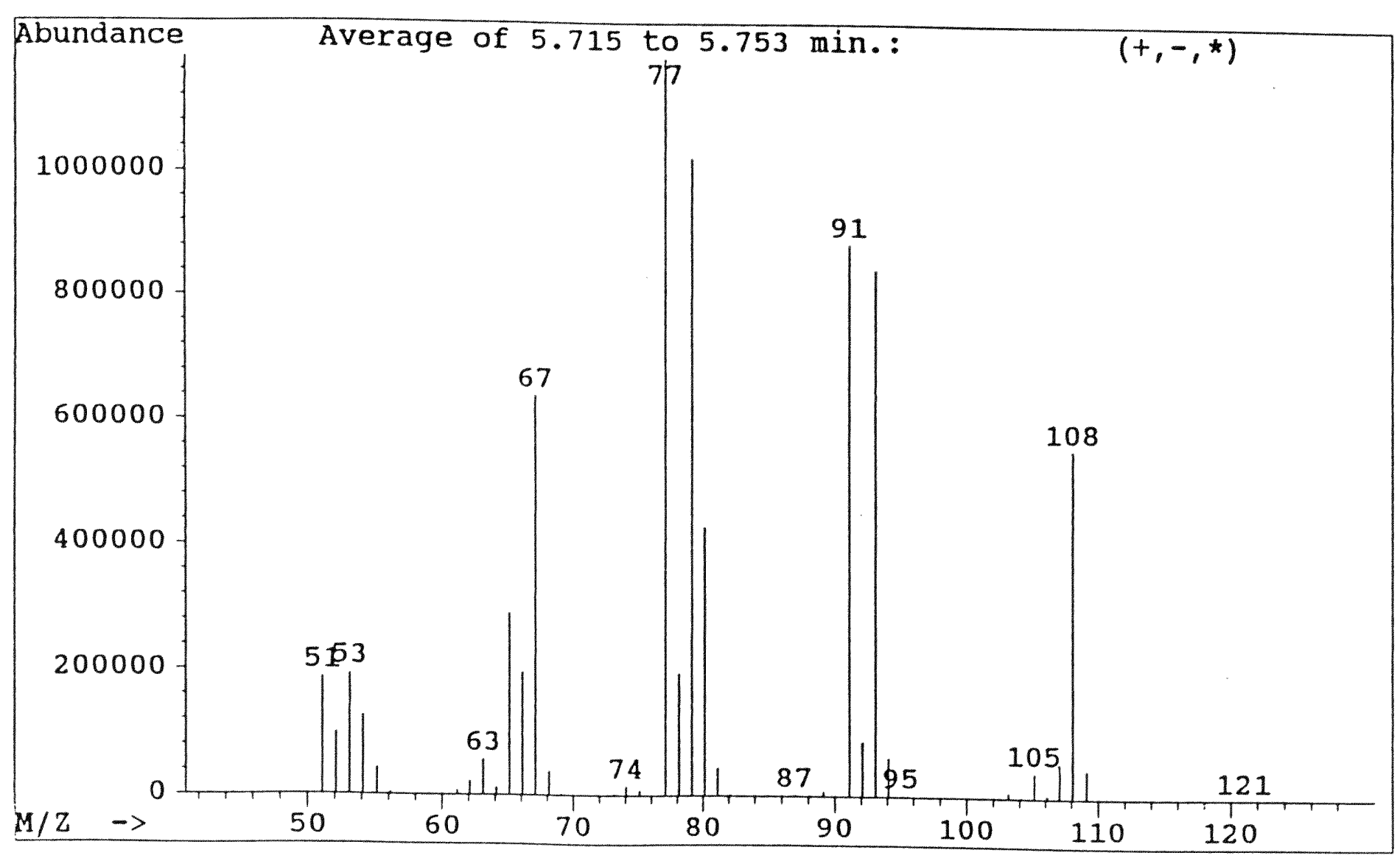




\subsection{SYNTHESIS AND CHARACTERIZATION OF trans-1,2- DICYCLOPROPYLETHYLENE}

In an attempt to selectively synthesize trans-1,2dicyclopropylethylene, a Wittig-Horner stereoselective modilication was adapted.50 The mechanism involves the nucleophilic addition of a resonance-stabilized phosphonate carbanion with a ketone or aldehyde. The stereoselectivity of the reaction is explained in terms of the relative energies of the betaine intermediates. The procedure typically gives an excellent stereospecific yield of the trans olefin. The reagents used are inexpensive and more reactive than the corresponding triarylphosphoranes.

The Schlosser and Christmann modification 51 of the Wittig reaction affords trans-selective olefin if equililibration of the erythro- and threo-betaines can be accelerated. The betaines, derived from non-stabilized phosphorus ylides, can redissociate into ylide and carbonyl compound resulting in trans-selective olefin formation. Interconversion of the diastereomeric betaine ylides is extremely rapid, and when treated with a strong base and proton donors at low temperature, betaine-LiX adducts (erythro- \& threo-) are regenerated with the equilibrium favoring the threo-betaine-LiX. Protonation then gives mainly the threo-betaine which yields the corresponding trans-olefin after treatment with potassium tbutoxide, as shown below. 


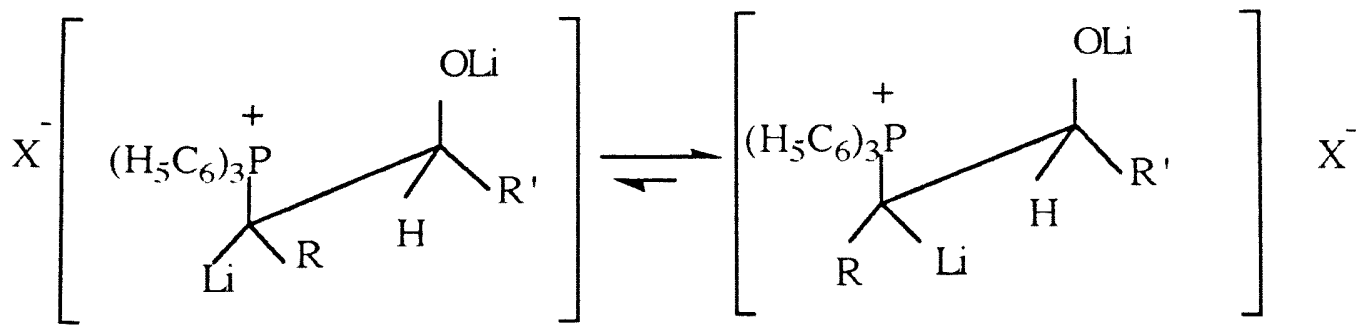

erythro-betaine

threo-betaine

Synthesis of trans-1,2-dicyclopropylethylene in a relatively high yield has been accomplished using lithium aluminum hydride as homogeneous hydrogenation catalyst, 52 which has been used successfully for the selective hydrogenation of alkynes and conjugated dienes to monoolefins Deuterium tracer experiments involving a diene have shown that the metal hydride and gaseous hydrogen each donate one hydrogen atom to the diene. Slaugh 52 observed the same mechanism of hydrogenation using 2-pentyne and lithium aluminum hydride. The alkyne was heated at $190{ }^{\circ} \mathrm{C}$ in a $\mathrm{H}_{2}$ atmosphere for $\sim 20 \mathrm{~min}$ and then cooled and quenched with excess $\mathrm{D}_{2} \mathrm{O}$. The high selectivity for monodeuterated pentene formation indicates that an intermediate adduct, lithium alkenylaluminum hydride, was formed which upon solvolysis produced 2-pentene.

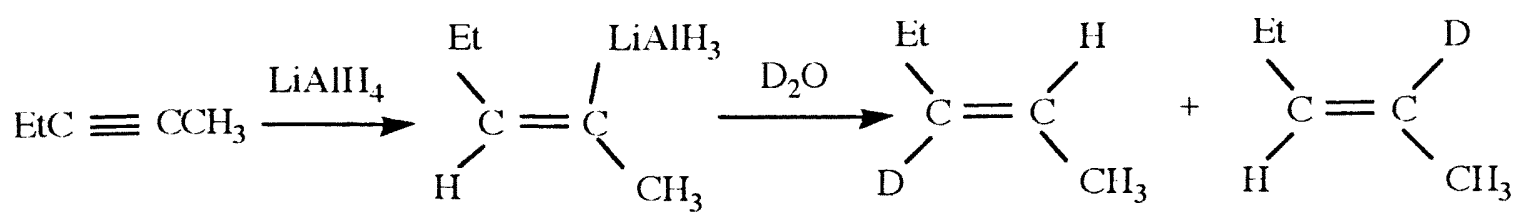




\subsubsection{SYNTHESIS OF trans-1,2-DICYCLOPROPYLETHYLENE \\ VIA WITTIG-HORNER REACTION}

\section{EXPERIMENTAL:}

Cyclopropylmethyl bromide (1.2 g, 8.3 mmoles) was transfered via syringe to a dry, $10 \mathrm{~mL}$ round bottom flask which had been purged with argon. An equimolar amount of triethyl phosphite (1.4 g, 8.3 mmoles) was added to the bromide, and the mixture was heated at reflux. The disappearance of the bromide was monitored by gas chromatography and was complete with 48 hours. The solution was magnetically stirred with a gentle purge of argon. The resulting mixture was allowed to cool to room temperature. Sodium methoxide (0.38 g, 8.3 mmoles) and DMF $(10 \mathrm{~mL})$ were placed in a clean, dry flask and combined with the phosphonate mixture from above. Cyclopropanecarboxaldehyde $(0.51 \mathrm{~g}, \quad 8.3 \mathrm{mmoles})$ was added dropwise to the mixture with constant stirring. No change was visible.

Since the expected change in color was not observed, 2 mmoles of n-butyllithium in hexane were added in an attempt to deprotonate the phosphonate and form the ylide. The color of the mixture changed slowly from a clear to a pale-yellow solution. It was then allowed to stir overnight at room temperature.

The reaction mixture was partitioned between equal volumes of hexane and water. The layers were separated, and the organic layer was washed successively with water and saturated $\mathrm{NaCl}$ 
solution. The organic layer was dried over anhydrous $\mathrm{MgSO}_{+}$for 5 min and filtered. The solvent was removed by fractional distillation.

Gas chromatographic analysis of the reaction mixture revealed the desired 1,2-dicyclopropylcthylenes were not present. The lack of product formation are likely due to the cyclopropyl groups acting as poor stabilizing groups for the phosphonate carbanion.

\subsection{2 tran s-SELECTIVE OLEFIN SYNTHESIS BY}

\section{REVERSIBILITY OF BETAINE FORMATION DURING THE WITTIG REACTION}

\section{EXPERIMENTAL:}

Triphenylphosphine (3.93 g, $15 \mathrm{mmoles})$, dry benzene $(50 \mathrm{~mL})$, and cyclopropylmethyl bromide $(2.03 \mathrm{~g}, 15 \mathrm{mmoles})$ were placed in a dry, $100 \mathrm{~mL}$ round bottom flask. The mixture was heated at reflux for 9 days with magnetic stirring and under an argon atmosphere. The cyclopropylmethylphosphonium salt was present in the form of an insoluble precipitate. The mixture was cooled to room temperature, and dry THF $(30 \mathrm{~mL})$ added to the phosphonium salt/benzene mixture. Upon dropwise addition of phenyllithium $\mathrm{mL}$ of a $1.8 \mathrm{M}$ solution in cyclohexane, 15 mmoles), the solution turned ycllow. The solution was cooled to $-78^{\circ} \mathrm{C}$ in a dry icc-acetone bath, and cyclopropanecarboxaldehyde (1.05 g, $15 \mathrm{mmoles})$ added to the cold mixture. After addition of the cyclopropanecarboxaldehyde, additional phenyllithium $(8.3 \mathrm{~mL}$ of a $1.8 \mathrm{M}$ solution in cyclohexane, 
15 mmoles) was slowly added, while maintaining the temperature at $-78{ }^{\circ} \mathrm{C}$. Anhydrous hydrogen chloride, $(16 \mathrm{~mL}$ of $1.0 \mathrm{M}$ solution in diethyl ether) and potassium t-butoxide $(22.5 \mathrm{~mL}$ of $1.0 \mathrm{M}$ in tetrahydrofuran) were added successively to the reaction mixture once it reached room temperature. Gas chromatographic analysis indicated the presence of a mixture of cis- and trans-1,2dicyclopropylethylenes in a $2: 1$ ratio. In an effort to increase the yield of the trans-1,2-dicyclopropylethylene a modification of the procedure was attempted in which addition of $\mathrm{HCl}$ and potassium $\mathrm{t}$ butoxide was accomplished at $-78^{\circ} \mathrm{C}$. Gas chromatography revealed an improvement in the cis/trans ratio to $1: 1$. This method is not applicable for the required selectivitics.

\subsubsection{SYNTHESIS OF trans-1,2-DICYCLOPROPYLETHYLENE USING LITHIUM ALUMINUM HYDRIDE, A HOMOGENEOUS HYDROGENATION CATALYST}

\section{EXPERIMENTAL:}

See the procedure described earlier for the synthesis of 1,2dicyclopropylethyne. Lithium aluminum hydride $(3.8 \mathrm{~g}, 0.1 \mathrm{~mol})$ was dissolved under argon in $140 \mathrm{~mL}$ of freshly-distilled THF and added to 1,2-dicyclopropylethyne (5) $(5.3 \mathrm{~g}, 50 \mathrm{mmol})$. The mixture was transfered to a steel autoclave, and the solution heated for 6 hours at $190-192^{\circ} \mathrm{C}$ and 30 atm $(441 \mathrm{psi})$ of $\mathrm{H}_{2}$, with continuous magnetic stirring. 
The contents of the autoclave were cooled to room temperature. After 12 hours at room temperature, the contents were poured over ice and acidified with $0.1 \mathrm{M}$ sulfuric acid. The mixture was extracted with four $50 \mathrm{~mL}$ portions of petroleum ether. The extracts were combined and dried over anhydrous $\mathrm{MgSO}_{+}$. After filtering off the $\mathrm{MgSO}_{4}$, the solvent was removed by distillation through a short Vigrcux column.

The distillate consisted predominantly of trans-1,2dicyclopropylethylene (7) (95\%) along with a small amount of cis1,2-dicyclopropylethylene $(5 \%)$ as detcrmined by capillary gas chromatography (Scheme IX). Although complete separation of the cis-isomer was unsuccessful, other impuritics were removed by preparative gas chromatography. Final charactcrization of the product was made by $1 \mathrm{H}$ - and $13 \mathrm{C}$-NMR spectroscopy (Figures XIX$\mathrm{XX)}$.

${ }^{1} \mathrm{H}-\mathrm{NMR}\left(300 \mathrm{MHz}, \mathrm{CDCl}_{3}\right) \delta(\mathrm{ppm}): 4.9-5.1 \quad(\mathrm{dd}, \quad 2 \mathrm{H}), \quad 1.2-1.3$ $(\mathrm{m}, \quad 2 \mathrm{H}), \quad 0.60-.070 \quad(\mathrm{dd}, \quad 4 \mathrm{H}), \quad 0.30-0.40 \quad(\mathrm{dd}, \quad 4 \mathrm{H}), \quad 13 \mathrm{C}-\mathrm{NMR}\left(\mathrm{CDCl}_{3}\right)$ : $133.01 \quad(d), 14.90 \quad(d), 7.77 \quad(t)$.

Scheme IX: Synthesis of trans-1,2-Dicyclopropylethylene

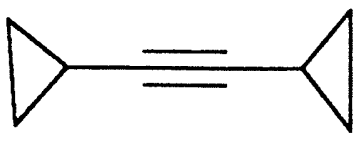

$$
\begin{aligned}
& \underset{190^{\circ} \mathrm{C} / 6 \mathrm{~h} .}{\mathrm{LiAlH}_{4}} \\
& 400 \mathrm{psi} \mathrm{H}
\end{aligned}
$$

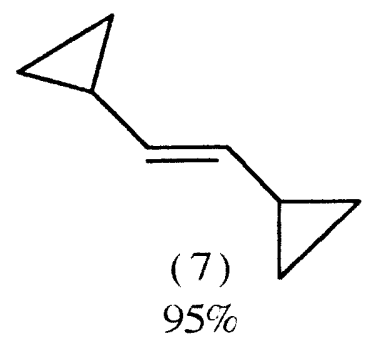


Figure XIX: ${ }^{1}$ II NMR SPECTRUM OF TRANS-DICYCLOPROPYLETHYLENE
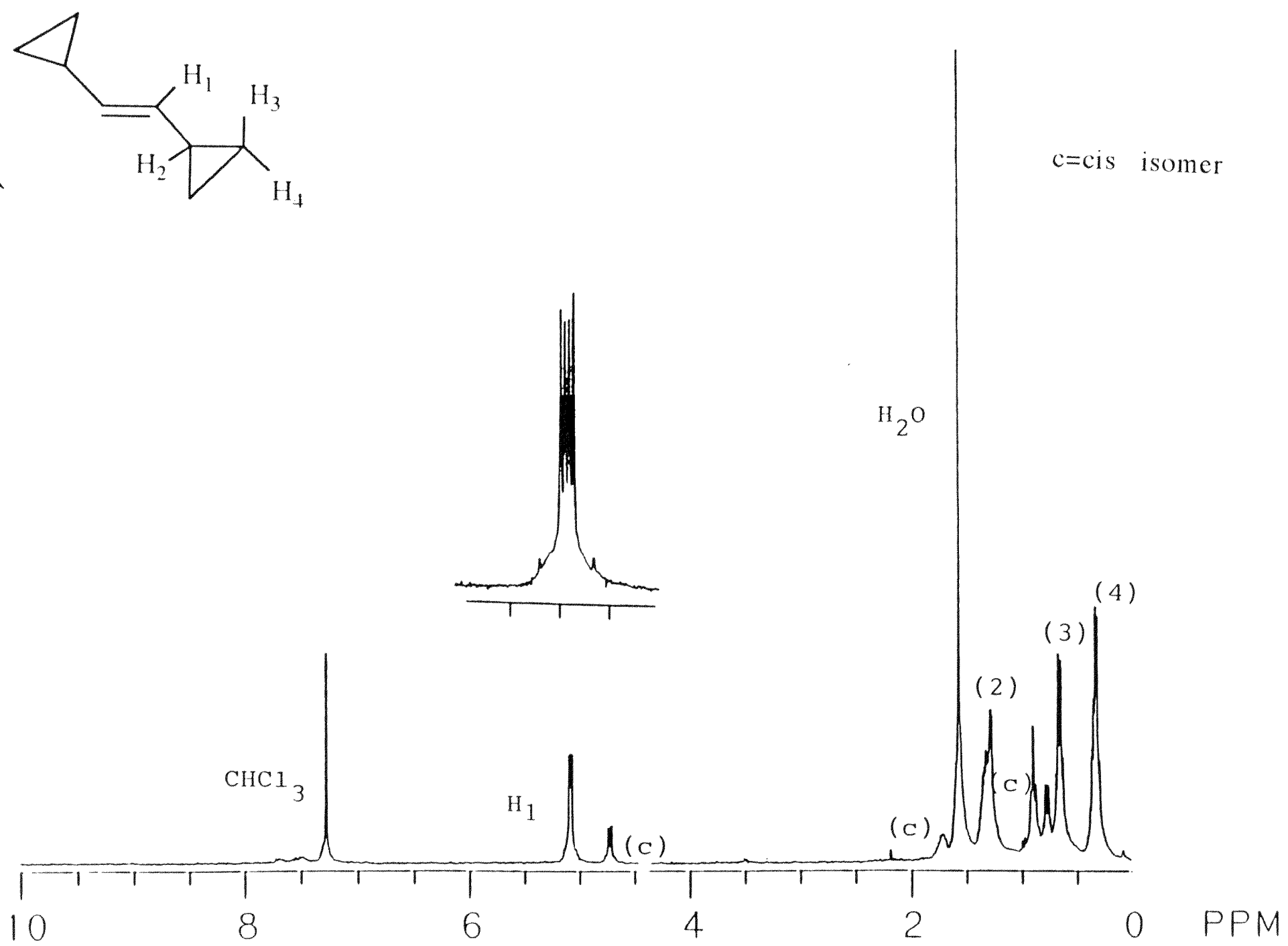
Figure XX: $\quad 13^{3} \mathrm{C}$ NMR SPECTRUM OF TRANS-DICYCLOPROPYLETHYLENE

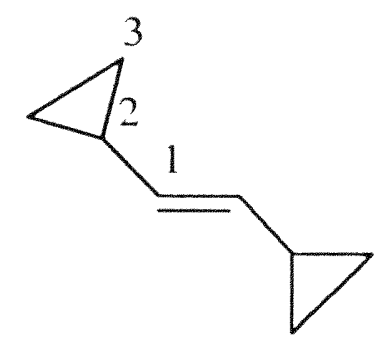

(c) $\mid(1)$

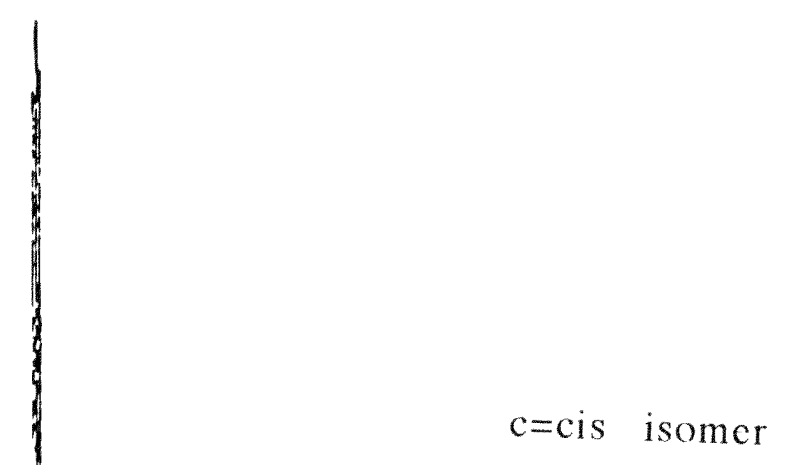

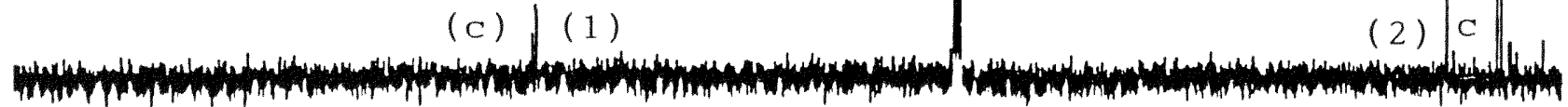

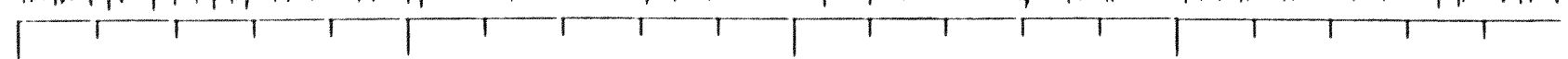

$\therefore 00$

150

100

50

o PPM 


\subsection{SYNTHESIS AND CHARACTERIZATION OF 2,4-DIMETHYL- 3-PENTENE}

2,4-Dimethyl-3-pentene was synthesized via Wittig reaction using 2,4-dimethyl-3-pentanone and triphenylmethylphosphonium iodide. After heating for 24 hours the yicld of alkene was $270 \%$ with $20 \%$ unreacted ketone. 53

\section{EXPERIMENTAL:}

Triphenylmethylphosphonium iodide $\quad\left(\begin{array}{llll}8.55 & \mathrm{~g}, & 21.2 \mathrm{mmoles})\end{array}\right.$ was weighed into a dry $100 \mathrm{~mL}$ round bottom flask, and $40 \mathrm{~mL}$ of dry tetrahydrofuran were cannulated into the reaction vessel. Phenyllithium in eyclohexane $(11.8 \mathrm{~mL}$ of a $1.8 \mathrm{M}$ solution, 21.2 mmoles) was added dropwise using an addition funnel. A color change from yellow to dark brown was observed. The mixture was stirred for $3 \mathrm{hrs}$ at room temperature. 2,4-Dimethyl-3-pentanone (Figure XXI) (2.4 g, 21.2 mmoles) was added slowly to the reaction mixture resulting in a color change from brown to gray. After stirring for an additional hour, a condenser was connected to the reaction vessel, and the mixture was heated at reflux for 24 hours.

The mixture was allowed to cool to room temperature and extracted successively with aqueuos of $5 \% \mathrm{HCl}, 10 \% \mathrm{NaHCO}_{3}$, and distilled water. The organic layer was separated and dried over anhydrous $\mathrm{MgSO}_{4}$, and the drying agent was removed by gravity filtration. The solvent was removed by fractional distillation. Gas 
chromatographic analysis indicated the presence of 1,1diisopropylethylene $(\sim 70 \%)$ (9), unreacted ketone ( 20\%) (Scheme X). The distillate was purified by column chromatography on silica gel (60-100 mesh, 2 in height and 2 in i.d.), using hexane as the eluant. The 1,1-diisopropylethylenc was identified and characterized by ${ }^{1} \mathrm{H}$ and 13 C-NMR spectroscopy (Figures XXII-XXIII).

${ }^{1} \mathrm{H}-\mathrm{NMR}\left(300 \mathrm{MHz}, \mathrm{CDCl}_{3}\right) \delta(\mathrm{ppm}): 4.736(\mathrm{~s}, 2 \mathrm{H}), 2.268$ (sept, $2 \mathrm{H}), 1.055-1.033(\mathrm{~d}, 12 \mathrm{H}) ; 13 \mathrm{C}-\mathrm{NMR}\left(\mathrm{CDCl}_{3}\right): 163.065(\mathrm{~s}), 103.578(\mathrm{t})$, 32.747 (d), 22.632 (q).

Scheme X: Synthesis of 1,1-Diisopropylethylene
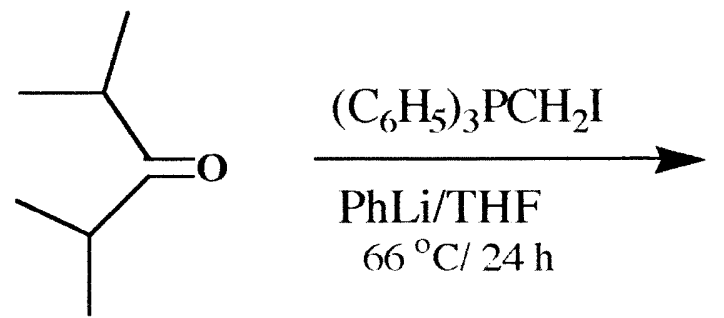

( 8 )

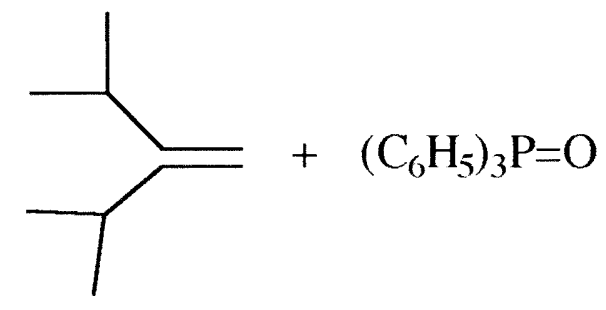

$70 \%$ 
Figure XXI: ${ }^{1} \mathrm{H}$ NMR SPECTRUM OF 2,4-DIMETHYL-3-PENTANONE

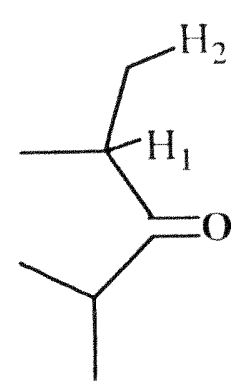

$\stackrel{a}{\omega}$ 
Figure XXII: ${ }^{1}$ H NMR SPECTRUM OF 1,1-DIISOPROPYLETHYLENE
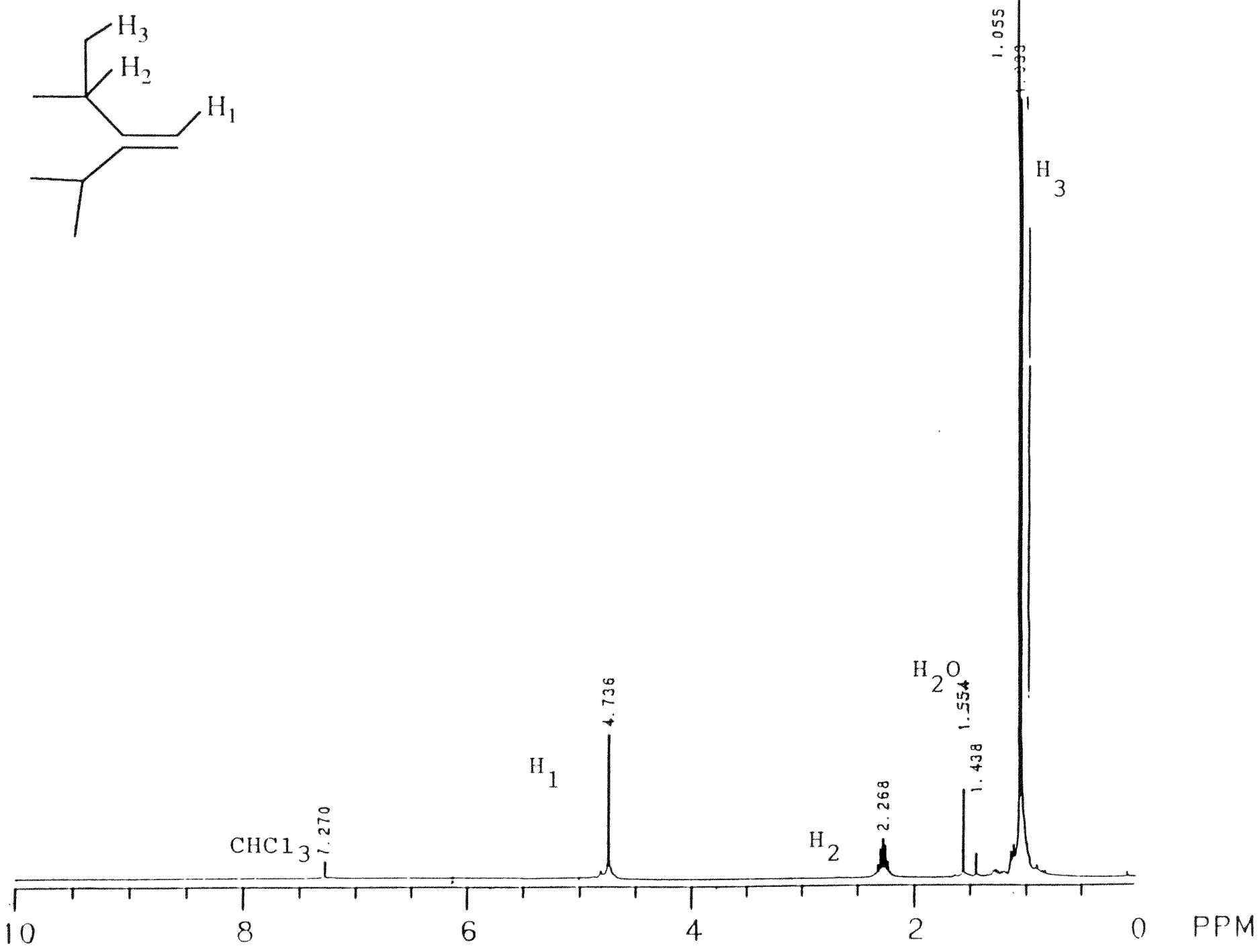
Figure XXIII: 13 C NMR SPECTRUM OF 1,1-DIISOPROPYLETHYLENE

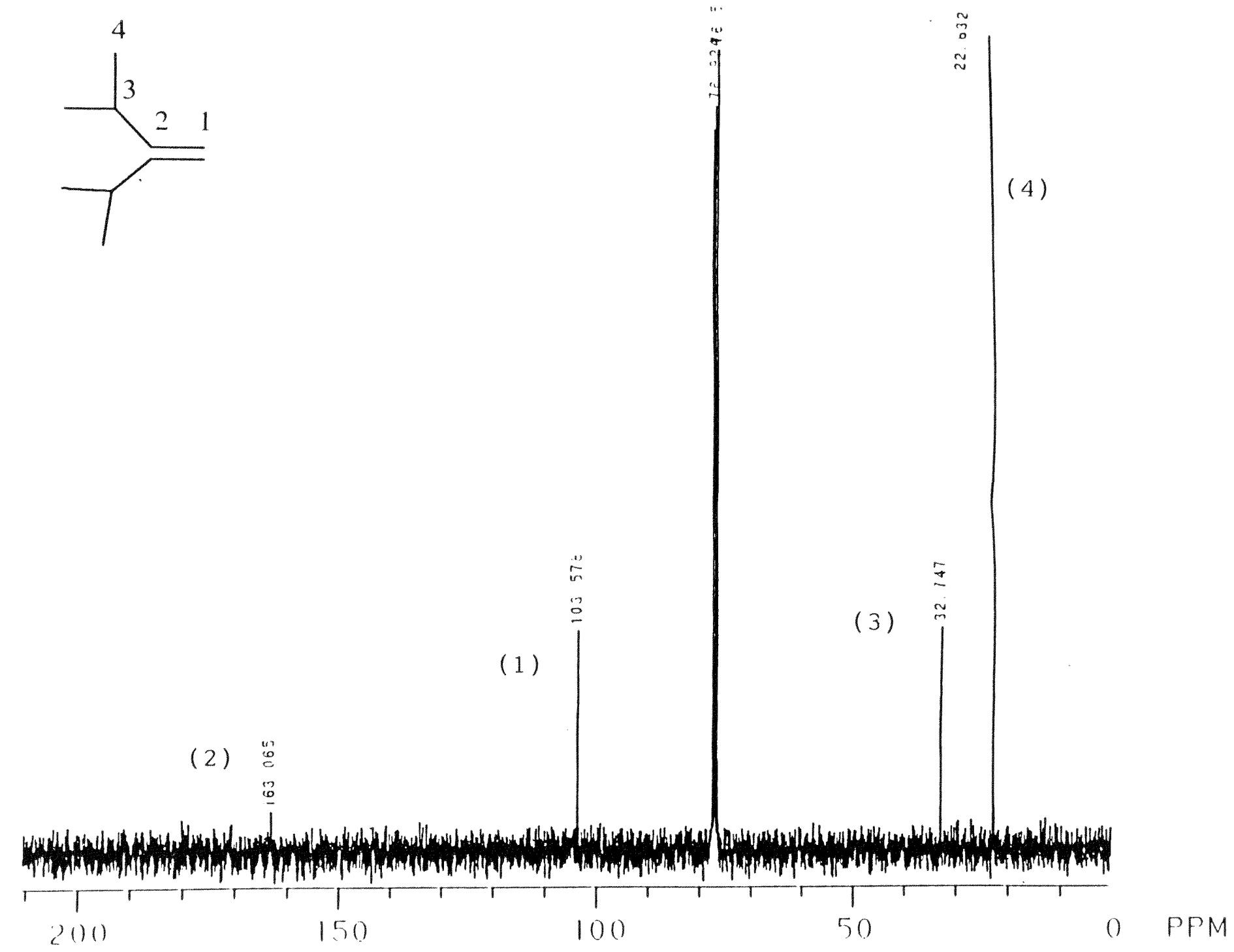


CHAPTER 3

REACTION STUDIES AND PRODUCT DISTRIBUTION 


\subsection{SINGLET OXYGEN REACTIONS WITH DICYCLOPROPYL SUBSTITUTED ETHYLENES}

\subsubsection{THE REACTION OF 1,1-DICYCLOPROPYLETHYLENE WITH SINGLET OXYGEN}

1,1-Dicyclopropylethylene was chosen as a probe to study the reaction mechanism of the electrophiles, ${ }^{O_{2}}$, MTAD, and TCNE with olefins. Since cyclopropyl groups have strong elcctron-donating abilities, cyclopropyl substituted ethylenes are cxpected to be clectron rich and be reactive toward clectrophiles. Cyclopropyl groups also stabilize positive charge through induction and can undergo skeletal rearrangement, in the case of $\alpha$ carbocation as discussed in chapter $I$, the presence of a cyclopropyl group are projected to stabilize a zwitterionic intermediate such that direct observation or trapping may be feasible, as shown below.

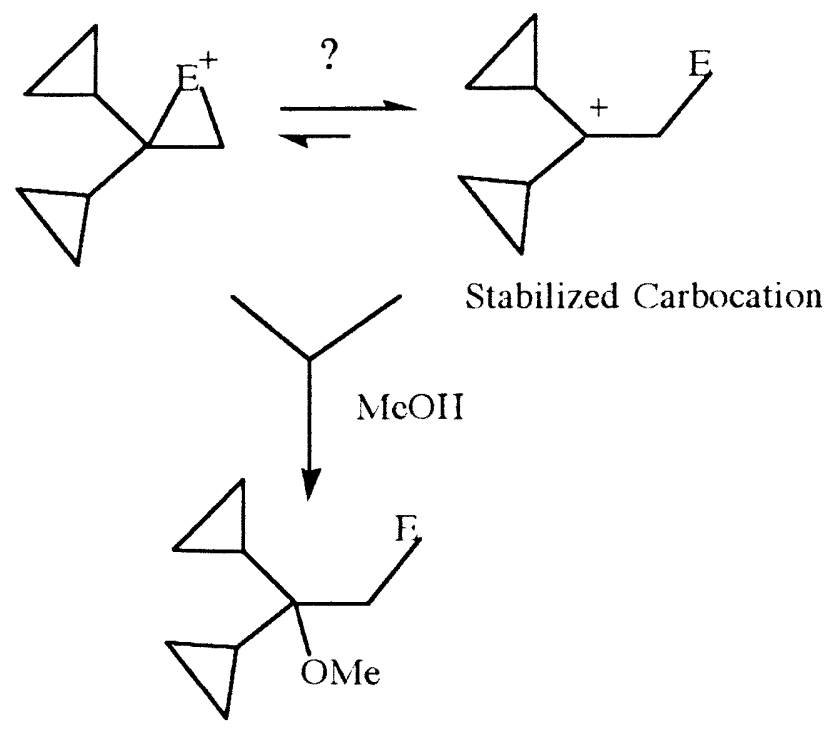


While the raction of 1,1-dicyclopropylethylene with 1 On has been reported, the scope of previous studies was limited to product studies. Frimer ${ }^{5+}$ reported the slow formation of dicyclopropyl ketone during photooxidation of 1,1-dicyclopropylethylene. We have studied the reaction in detail including the investigation of solvent effects.

\section{GENERAL PHOTOLYSIS PROCEDURE:}

Dicyclopropyl substituted ethylene (1,1-dicyclopropylethylene or cis-1,2-dicyclopropylethylene $)(0.010 \mathrm{~mL}), 0.2 \mathrm{~mL}$ of singlet oxygen photosensitizer solution $\left(10^{-4} \mathrm{M} \mathrm{ZnTPP}\right.$ in $\left.\mathrm{CDCl}_{3}\right)$ and $\sim 1 \mathrm{~mL}$ of $\mathrm{CDCl}_{3}$ were placed in a NMR tube. The NMR tube was placed in a windowed Dewar flask maintaining a slow, steady flow of oxygen through the solution and a temperature of $0{ }^{\circ} \mathrm{C}$. A 150 watt Xenon lamp, Oriel Model 68806 was used to irradiate the samples. A potassium cromate solution $\left(\begin{array}{lllll}5.0 & \mathrm{x} & 10^{-4} & \mathrm{M}\end{array}\right)$ was used as a chemical filter and pyrex glass were used to eliminate short UV wavelengths $(<312.6 \mathrm{~nm})$ and to ensure no direct excitation of the olefin. Gas chromatography and $1_{\text {H-NMR }}$ were used to monitor the reaction. No detectable changes were observed in the starting materials after 12 hrs of irradiation. 


\section{RESULTS:}

The cxpected products are shown below. We found that photooxidation of 1,1-dicyclopropylethylene in the presence of $\mathrm{ZnTPP}, \mathrm{O}_{2}$ and at $0{ }^{\circ} \mathrm{C}$ yiclded no appreciable reactions contrary to Frimer's reports. 54

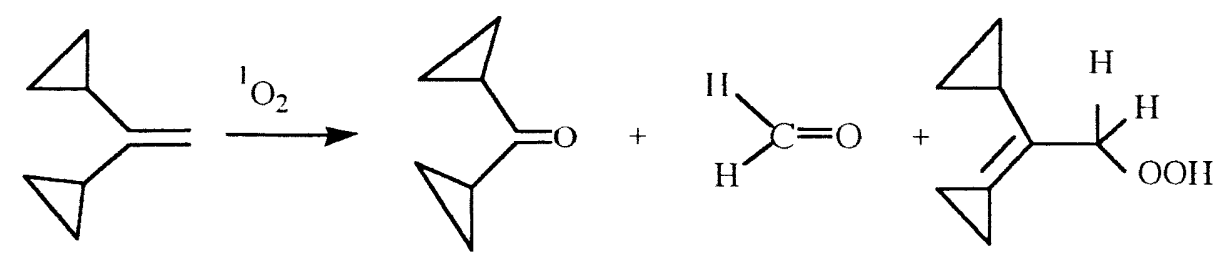

To confirm singlet oxygen formation during the photooxidation, tetramethylenc (TME) (10 uL) was added to the reaction mixture. TME is commonly used as a diagnostic reagent to determine if singlet oxygen is being formed and/or quenched. After one hour of irradiation 1 H-NMR (Figures XXIV \& XXV) showed the presence of the characteristic hydroperoxide confirming the generation of $1 \mathrm{O} 2$ in solution under our standard reaction conditions.
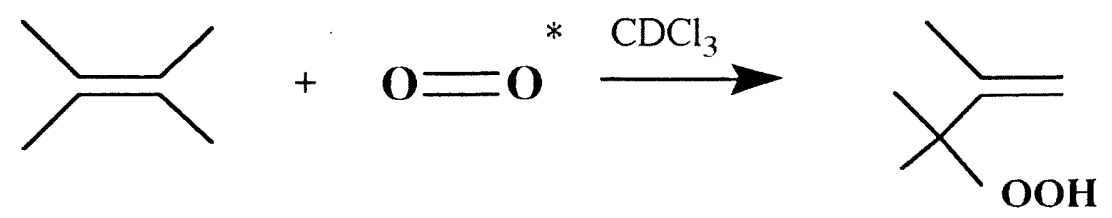

Reaction of 1,1-dicyclopropylcthylene with singlet oxygen has becn reported by Frimer to be quite slow $\left(k_{\mathrm{rel}}=\mathrm{k}_{\mathrm{olefin}} / \mathrm{k}\right.$ TME $=3.5 \mathrm{x}$ $10^{-5}$ ), with dicyclopropyl ketone (25\% yicld) and a polymer (75\%) as 
the products. He suggests the ketone is not product of the dioxetanc or allylic hydroperoxide from ${ }^{1} \mathrm{O}_{2}$ since the addition of a large excess of either the dioxetane trap diphenyl sulfide or the hydroperoxide reducing agent triphenyl phosphite does not affect the product yicld. A frec-radical process appears to be involved in the formation of the ketone. Our results demonstrate the reaction involving $\mathrm{l}_{\mathrm{O}}$ is negligible under our experimental conditions.

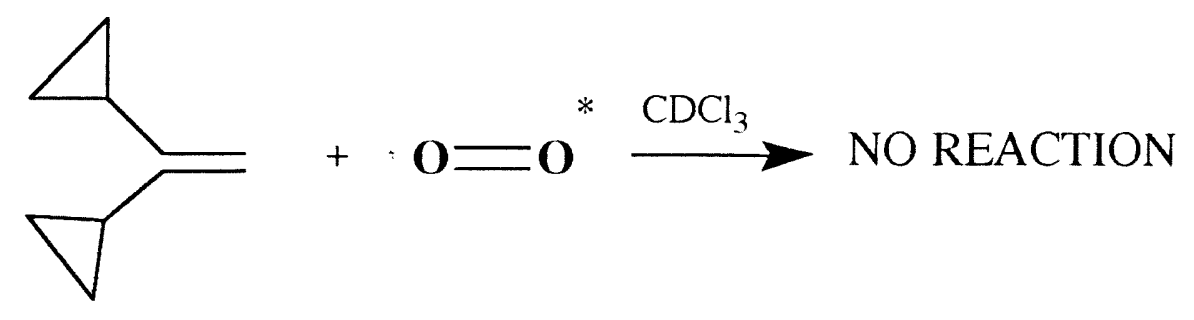

Frimer results are probably duc to Type I oxidation which involves radicals or radical ions by hydrogen atom or electron transfer. Possibly the formation and rcarrangement of the hydroperoxide are as shown below.

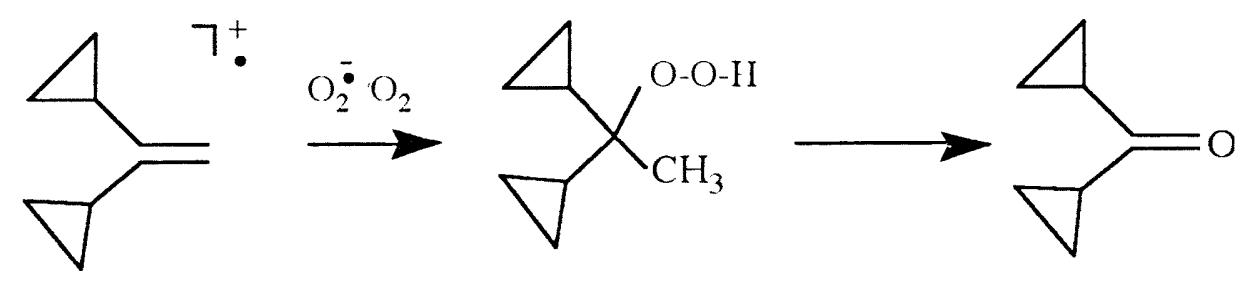

In our study the solvent was chloroform and the sensitizer was ZnTPP. While Frimer used acetonitrile as solvent and as photosensitizer methylene blue $10^{-3} \mathrm{M}$. Since methylene blue is known to produce more Type II reactions this can influence the relative differences in these studies. 
FIGURE XXIV: ${ }^{1}$ H NMR SPECTRUM OF TIE REACTION OF 1,1-DICYCLOPROPYLETHYLENE WITH SINGLET OXYGEN IN THE PRESENCE OF TETRAMETHYLENE
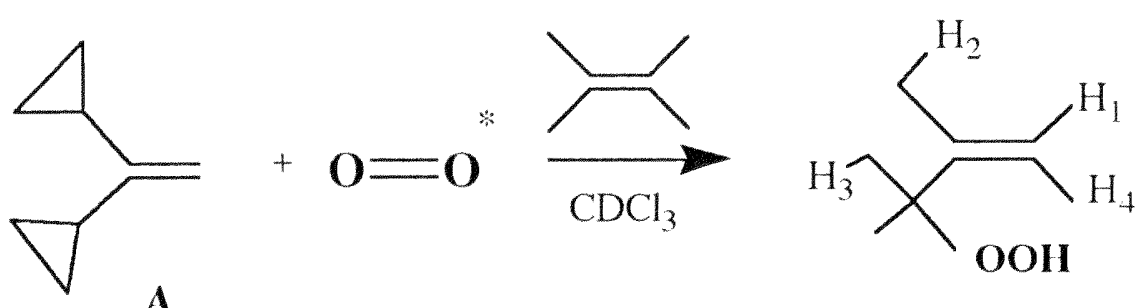

A

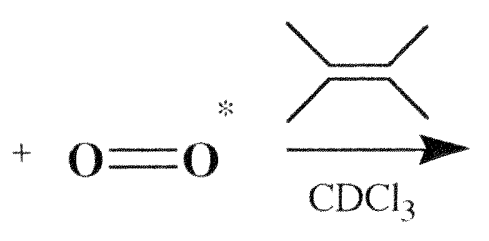


FIGURE XXV: 13 C NMR SPECTRUM OF THE THE REACTION OF 1,1DICYCLOPROPYLETHYLENE WITH SINGLET OXYGEN IN THE PRESENCE OF TETRAMETHYLENE
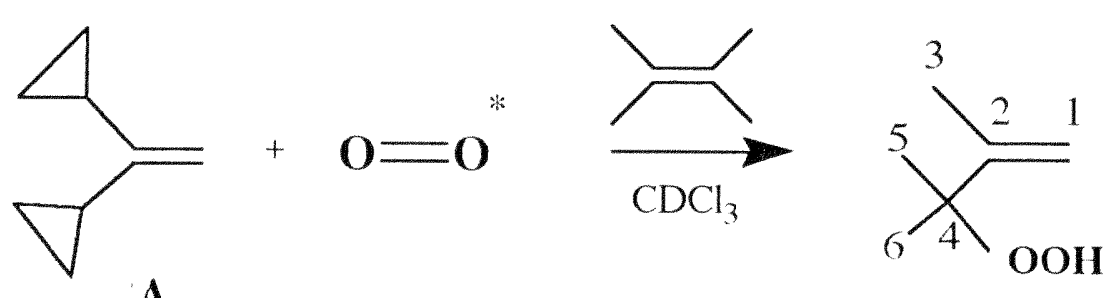

(A)

(1) $\stackrel{\infty}{\stackrel{\infty}{\infty}}$

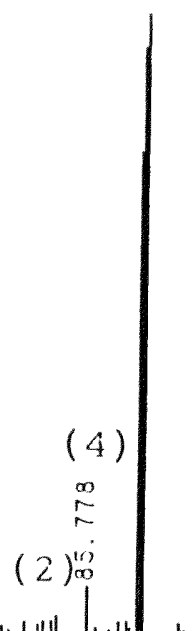

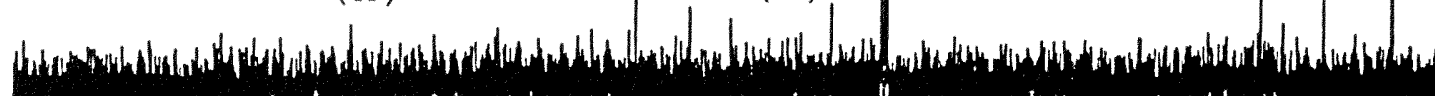

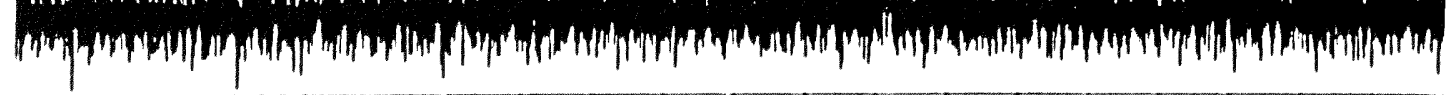

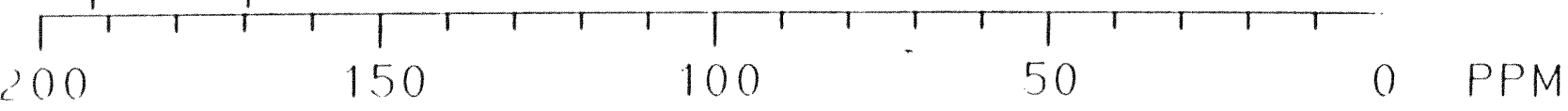




\subsubsection{THE REACTION OF $c$ is-DICYCLOPROPYLETHYLENE WITH SINGLET OXYGEN}

cis-Dicyclopropylethylenc was used to probe the stereochemical consequences of the reactions of electrophiles with alkenes. Since two olcfinic carbons are equally substituted, they are not subject to electronic and steric effects related to regiochemistry of these reactions.

If the reaction of cis-dicyclopropylethylene with singlet oxygen involves a concerted pathway or takes place through a close intermediate, stercospecific products are expected. If the reaction involves non-concerted reaction pathways involving an open intermediate, the stereochemistry would not be maintained in the products. Dioxctane products are generally unstable at room temperature and cleave to give carbonyl compounds which do not provide insight with regard to the stereochemistry of the initial dioxetane product. Low temperature NMR maybe required to observe the stereochemistry of the products.

Orfanopoulos 55 studied the photooxygenation of cis-and trans2-butenes to probe the proposed perepoxide intermediate. These studies provide strong support for a perepoxide, or the kinetically equivalent exciplex and suggest its formation may be reversible. cis2-Butene-1,1,1-d3 was found to have a product isotope effect $\left(\mathrm{k}_{\mathrm{H}} / \mathrm{k}_{\mathrm{D}}=1.38\right)$, and it was also more reactive than the trans-isomer. The small kinetic and substantial product isotope effects strongly 
suggest the rate-determining formation of an intermediatc without cleavage of a C-H bond corresponding to the perepoxide or a $n$ cxciplex intermediate.

\section{RESULTS:}

cis-1,2-Dicyclopropylethylene docs not undergo photooxidation upon irradiation for $-12 \mathrm{hrs}$ in the presence of ZnTPP as sensitizer, $\mathrm{CDCl}_{3}$ as solvent and the temperature was $0^{\circ} \mathrm{C}$. The presence of $1 \mathrm{O}$ formation was confirmed using TME. A number of factors maybe responsible for the lack of reaction. The cis-isomer is expected to stabilize the perepoxide intermediate (cis effect) as shown below.

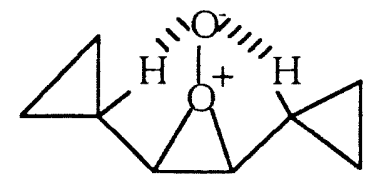

If formed the perepoxide is expected to yield the ene and/or $2+2$ products while both the ene and $2+2$ are strained, the formation of the hydroperoxide, ene product requires the formation of a highly strained cyclopropylidene.

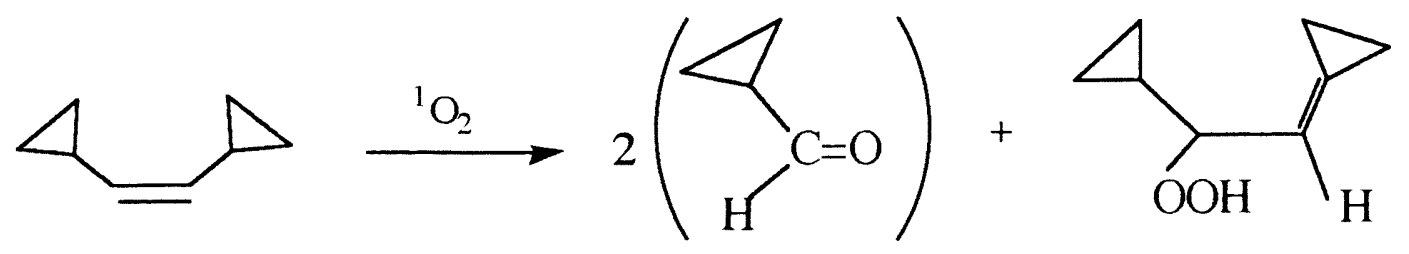


If the perepoxide is formed but falls apart to starting materials one would not observe the formation of products despite physical quenching of ${ }^{1} \mathrm{O}_{2}$. Studies exploring the quenching of $\mathrm{O}_{2} \mathrm{~b} y$ eyclopropylethylenes are planned.

\subsubsection{THE REACTION OF 1,1-DISOPROPYLETHYLENE WITH SINGLET OXYGEN}

We chose to use 1,1-diisopropylethylene to further probe the reactivity of 1 O towards disubstituted olefins. The photooxidation of 1,1-dicyclopropylethylene was negligible, presumably the result of steric factors and the high activation energy expected to yield strained products. Isopropyl groups of 1,1-diisopropylethylene may have slightly greater steric hinderance than the cyclopropyl groups of 1,1-dicyclopropylethylene. The expected ene product is dramatically less strained than the cyclopropylidene as shown below.

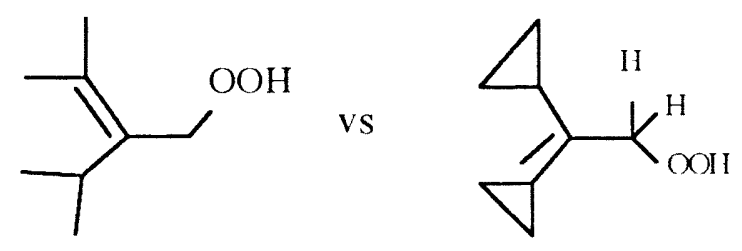

\section{RESULTS:}

Our results have shown that 1,1-diisopropylethylene does not react singlet oxygen under our experimental conditions. No change could be detected in the reaction mixture using NMR spectroscopy 
and gas chromatography. Similar compounds such as 2-isopropyl-1butene react with singlet oxygen very slowly with a $\mathrm{k}_{\mathrm{rel}}=8.2 \times 10^{-5}$ as observed by Kopecky.56 This result demonstrated the poor reactivity of this compound. 1,1-Diisopropylethylene is probably unreactive because of the geminal isopropyl groups that prevent the approach of the electrophile to the double bond. 


\subsection{REACTIONS OF 4-METHYL-1,2,4-TRIAZOLINE-3,5-DIONE (MTAD) WITH DISUBSTITUTED ETHYLENES}

\subsubsection{REACTION OF 4-METHYL-1,2,4-TRIAZOLINE-3,5- DIONE (MTAD) WITH 1,1-DICYCLOPROPYLETHYLENE}

4-Methyl-1,2,4-triazoline-3,5-dione is known to be a stronger electrophile than singlet oxygen and TCNE, and generally reacts readily with olefins, including disubstituted olefins. The expected products of 1,1-dicyclopropylethylene and MTAD are 2+2 and ene type products.
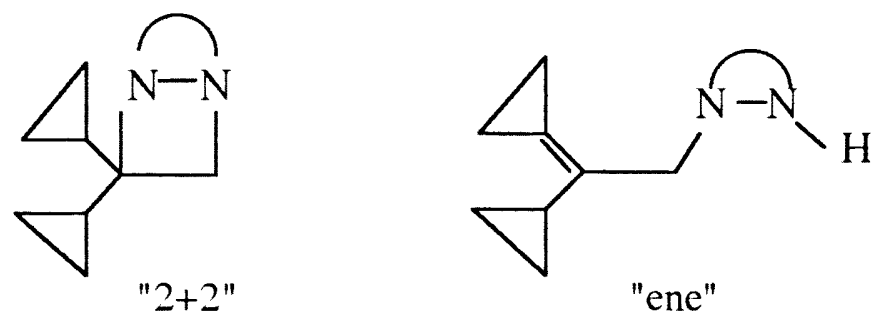

$$
\mathrm{N}_{\mathrm{N}}=\mathrm{N}_{\mathrm{N}-\mathrm{C}_{\mathrm{O}}}^{\mathrm{N}} \mathrm{C}^{\mathrm{N}} \mathrm{CH}_{3}
$$

Several research groups have reported the involvement of an aziridinium imide in such reactions. Foote 25 has recently proposed the equilibrium between the open and closed zwitterion forms, in the reaction of N-substituted-1,2,4-triazoline-3,5-diones and transcyclooctene. 


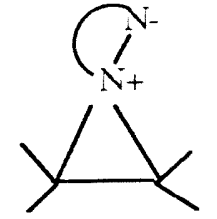

Closed Zwitterion

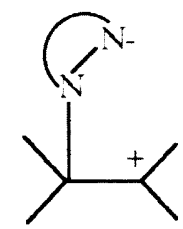

Open Zwitterion

AZI

If the closed zwitterion, $A Z I$, is involved in the reaction and if electronic factors direct the attack of the nucleophile, addition at the more substituted carbon is expected (Markonikov addition). An open zwitterion is expected to yield similar result. On the other hand, if steric hinderance is the predominant factor directing the nucleophilic addition to the AZI, addition at the less substituted carbon would be observed and will provide strong evidence for the close zwitterion as intermediate.

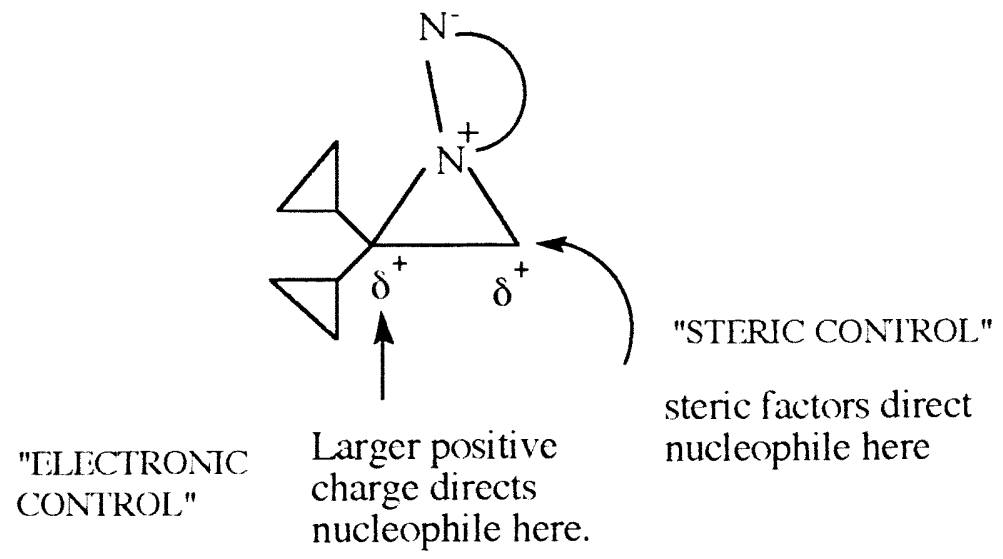

Low temperature NMR studies are used in an attempt to observe the AZI directly and methanol is used to trap zwitterionic intermediates. Anti-Markonikov's addition of methanol/RTAD across the double bond provides strong evidence to the involvement of the 
AZI, while Markonikov's adducts can be rationalized as products from both the close and open zwitterions.

\section{GENERAL PROCEDURE:}

Approximately $20 \mathrm{uL}$ of a pure olefin $(1,1-$ dicyclopropylethylene, cis-1,2-dicyclopropylethylene, trans-1,2dicyclopropylethylene and 1,1-diisopropylethylene) collected by prep-GC were mixed with $1 \mathrm{~mL}$ of $\mathrm{CDCl}_{3}$ in an NMR tube kept at $0{ }^{\circ} \mathrm{C}$ with ice-water in a Dewar. An equimolar amount of MTAD (from Aldrich Co.) or a solution of $\mathrm{MTAD}$ in $\mathrm{CDCl}_{3}$ was added to the alkene solution. Upon addition of $\mathrm{MTAD}$ in $\mathrm{CDCl}_{3}$, a red colored solution, to the alkene, the mixture has a red color as the electrophile initially, after $5 \mathrm{~min}$ the red color dissappeared completely indicating that the reaction was complete. ${ }^{1} \mathrm{H}-,{ }^{13} \mathrm{C}-\mathrm{NMR}$ and the Attached Proton Test (APT) were used to identily and characterize the reaction products.

\section{RESULTS:}

The reaction of 1,1-dicyclopropylethylene and MTAD yielded two products, presumably the $2+2$ and the ene products. Gas chromatography was used to monitor the dissapearance of the reactants and the appearance of the products. The mixture of products were identified by NMR and percent yield determined by G.C. The diazetidine, $2+2$ product was formed in $75 \%$ and the other product in $25 \%$ (Figures XXVI-XXVIII). 
FIGURE XXVI: 111 NMIR SPECTRUM OF REACTION OF 1,1-DICYCLOPROPYLETIYLENE WITII MTAD

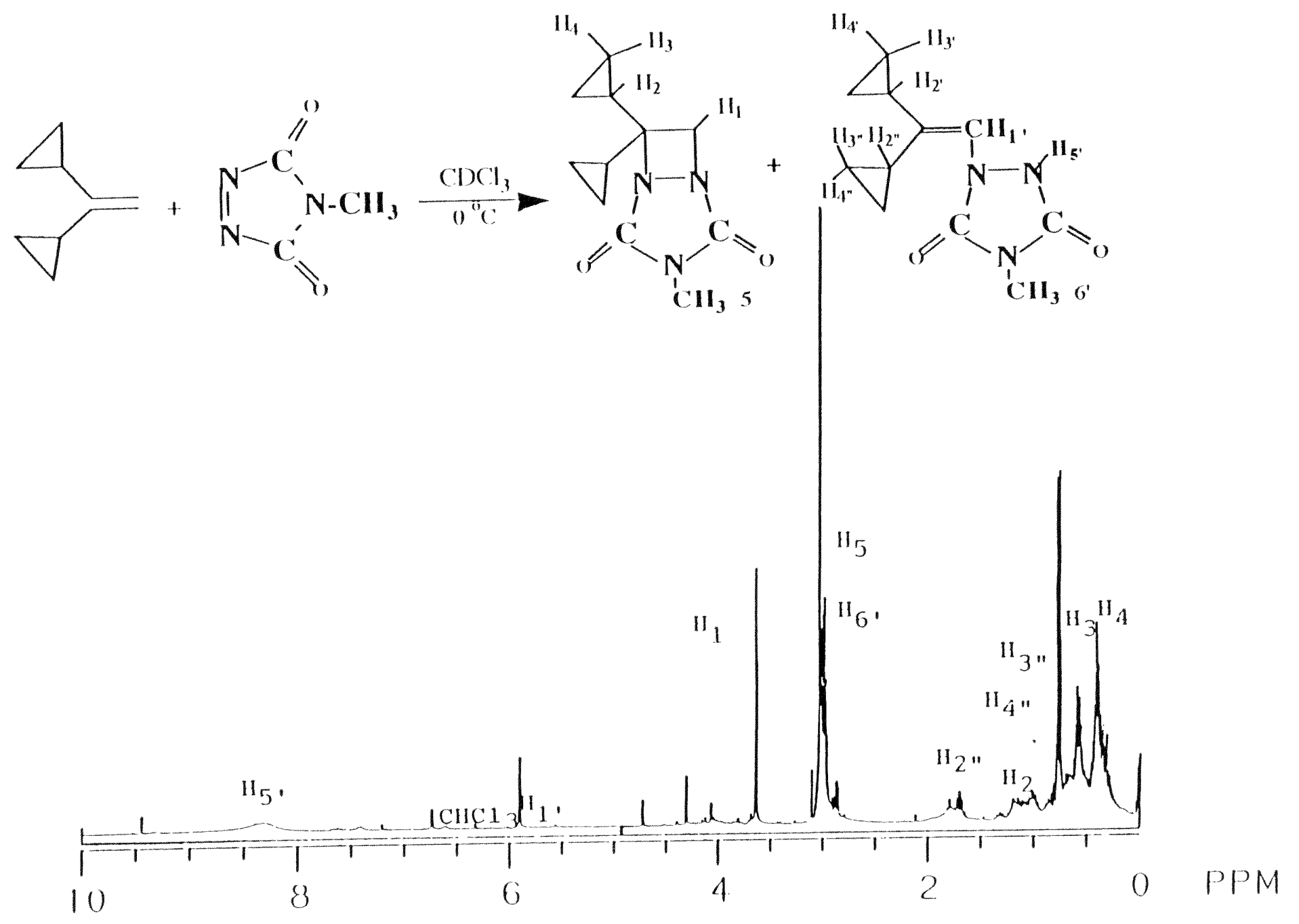


FIGURE XXVII: 1 3C NMR SPECTRUM OF REACTION OF 1,1-DICYCLOPROPYLETHYLENE WITH MTAD

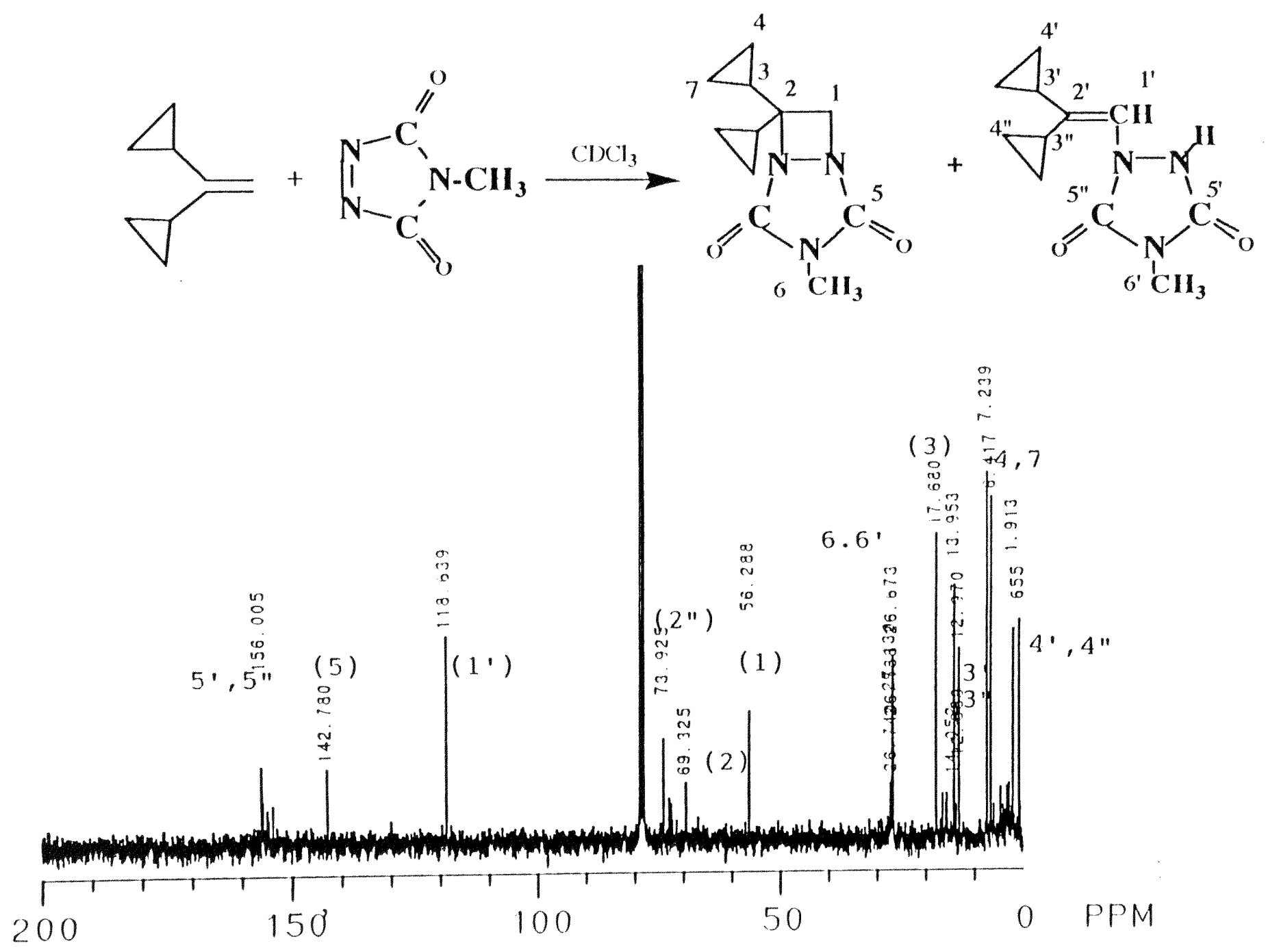


FIGURE XXVIII: ATTACHED PROTON TEST SPECTRUM OF REACTION OF 1,1DICYCLOPROPYLETHYLENE WITH MTAD
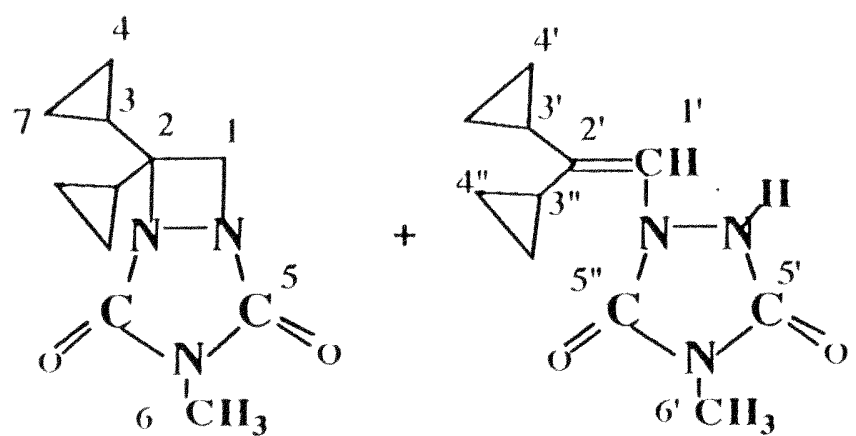

$$
6 \mathrm{CHI}_{3}
$$

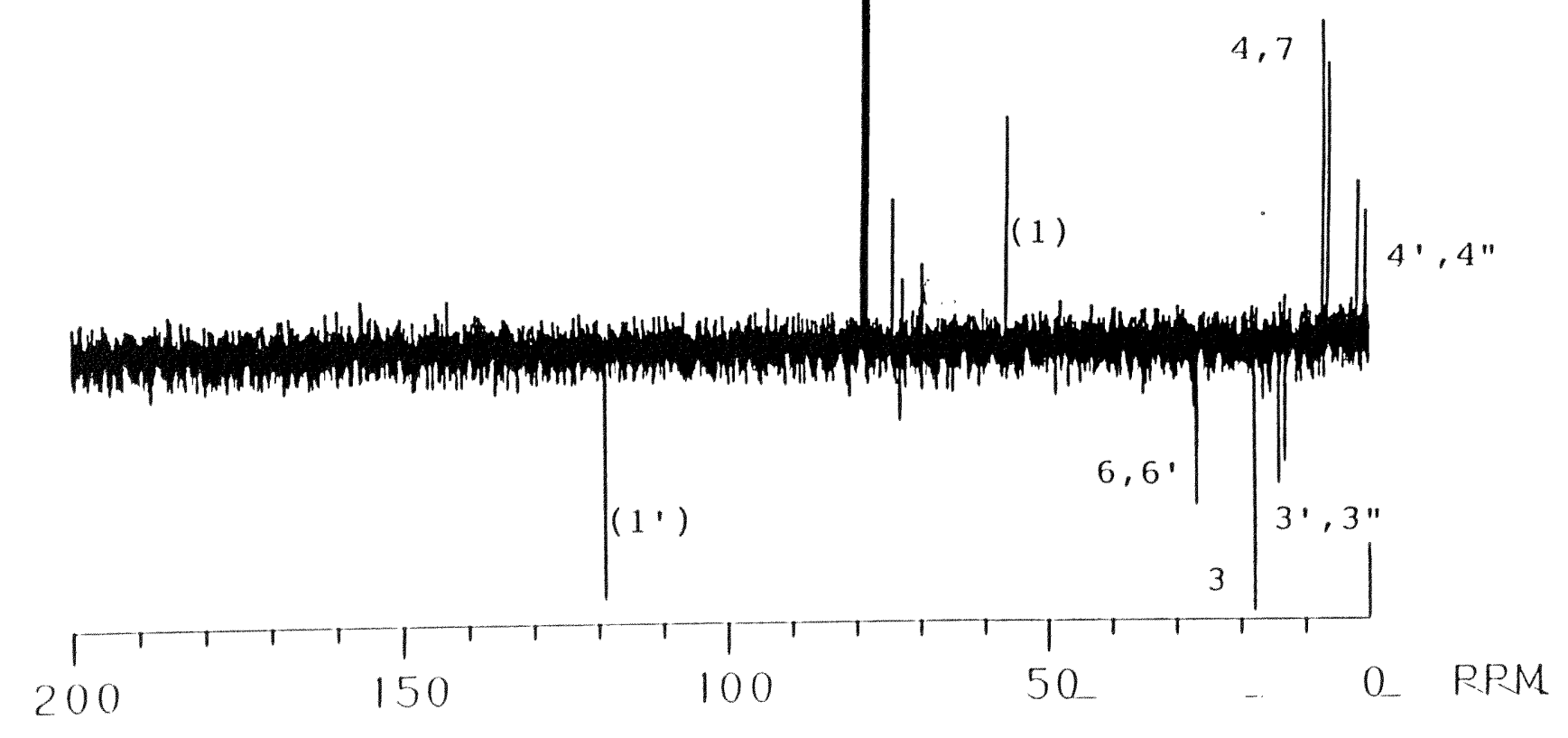


The diazctidine, $2+2$ product was purilied from the reaction mixture using column chromatography on silica gel (200-340 mesh, 5 in length, -1.5 in internal diameter), using mixtures of ethyl acetate and hexanc $2: 1$ as cluant and characterized by ${ }^{1} \mathrm{H}-\mathrm{NMR}$ and GC-MS (Figure XXIX \& XXX). ${ }^{1} \mathrm{H}-\mathrm{NMR}\left(300 \mathrm{MHz}, \mathrm{CDCl}_{3}\right) \delta(\mathrm{ppm}): 3.65$ (s, 2H), $3.2(\mathrm{~s}, 3 \mathrm{H}), 0.85(\mathrm{~m}, 2 \mathrm{H}), 0.50(\mathrm{~m}, 4 \mathrm{H}), 0.45(\mathrm{~m}, 4 \mathrm{H})$.

After isolation of the expected ene product, NMR peaks did not coincide with the cxpected structurc, a peak obscrved by $1^{\mathrm{H}-}$ and $13 \mathrm{C}-\mathrm{NMR}$ and APT in the olefinic area suggests the presence of a $\mathrm{CH}$ and the traditional ene product does not have any olefinic proton. W e suggest after analysis of the spectrum the presence of a product which in an effort to relieve the strain in its structure rearranges by a [1,3] hydride shift. ${ }^{1} \mathrm{H}-\mathrm{NMR}\left(300 \mathrm{MHz}, \mathrm{CDCl}_{3}\right) \&$ (ppm): 7.95 (b, 1H), $6.02(\mathrm{~s}, 1 \mathrm{H}), 3.2(\mathrm{~s}, 3 \mathrm{H}), 1.5(\mathrm{~m}, 1 \mathrm{H}), 1.2(\mathrm{~m}, 1 \mathrm{H})$ (Figures XXXI \& XXXII).

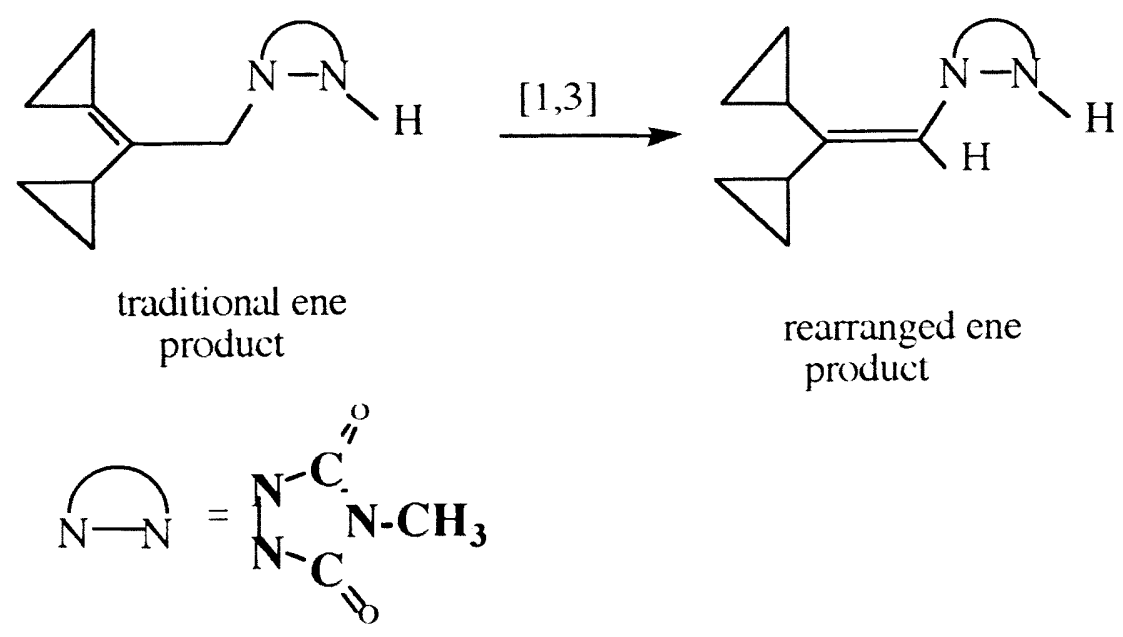


FIGURE XXIX: ${ }^{1}$ H NMR SPECTRUM OF CYCLOADDITION PRODUCT OF REACTION OF 1,1DICYCLOPROPYLETHYLENE WITH MTAD
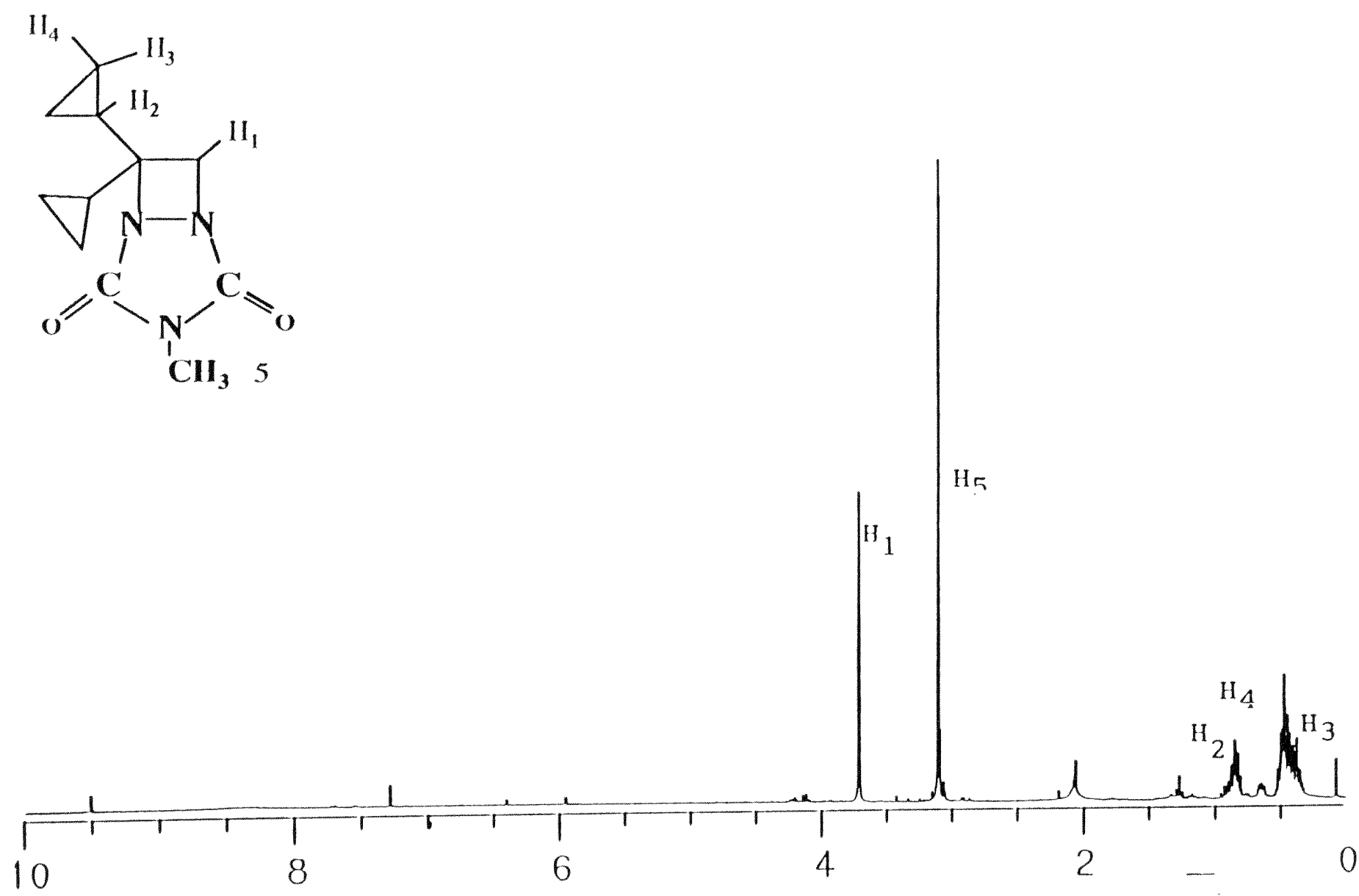


\section{FIGURE XXX: MASS SPECTRUM OF CYCLOADDITION PRODUCT OF REACTION OF 1,1. DICYCLOPROPYLETHYLENE WITH MTAD}

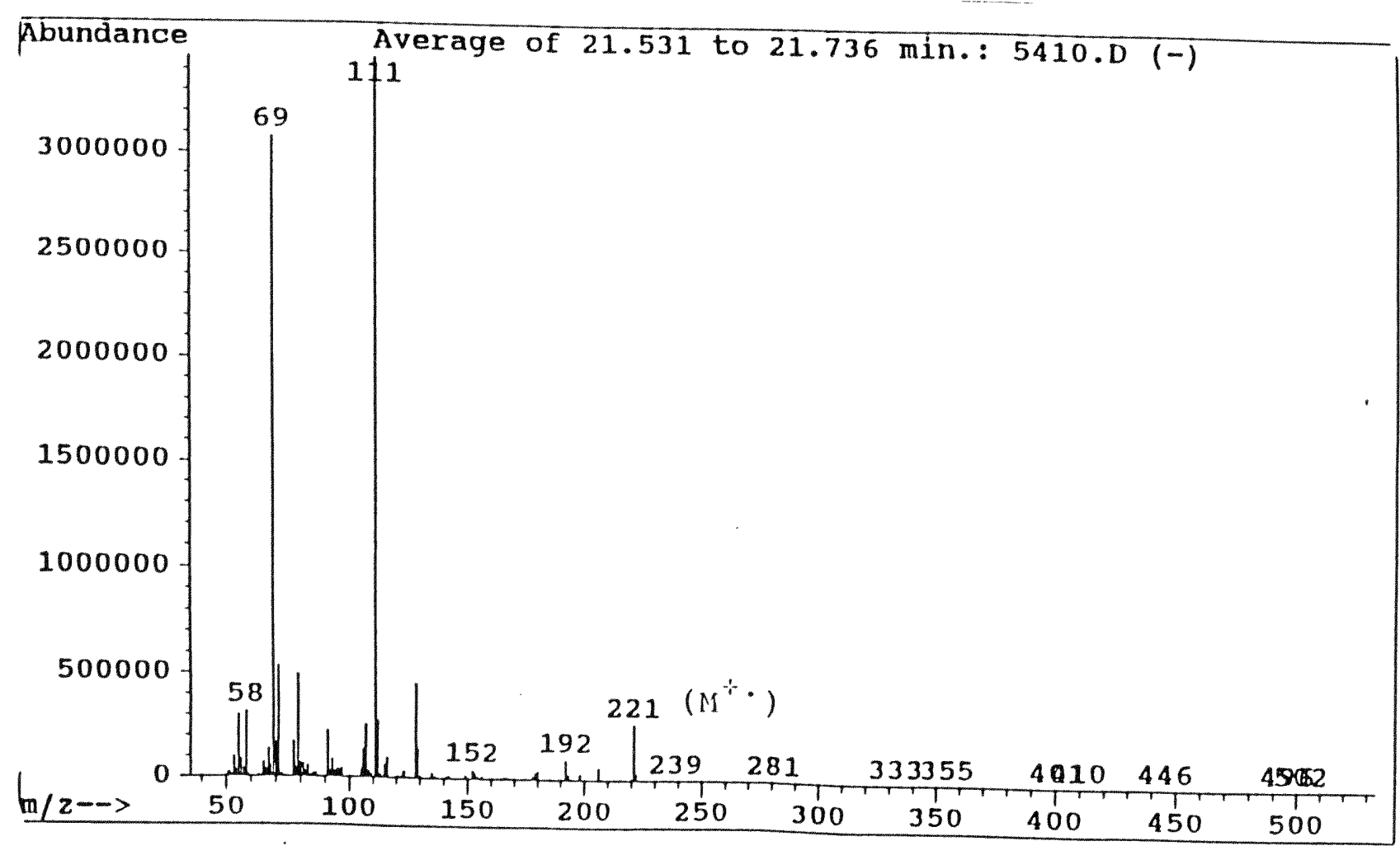


FIGURE XXXI: ${ }^{1}$ H NMR SPECTRUM OF ENE PRODUCT OF REACTION OF 1,1DICYCLOPROPYLETHYLENE WITH MTAD
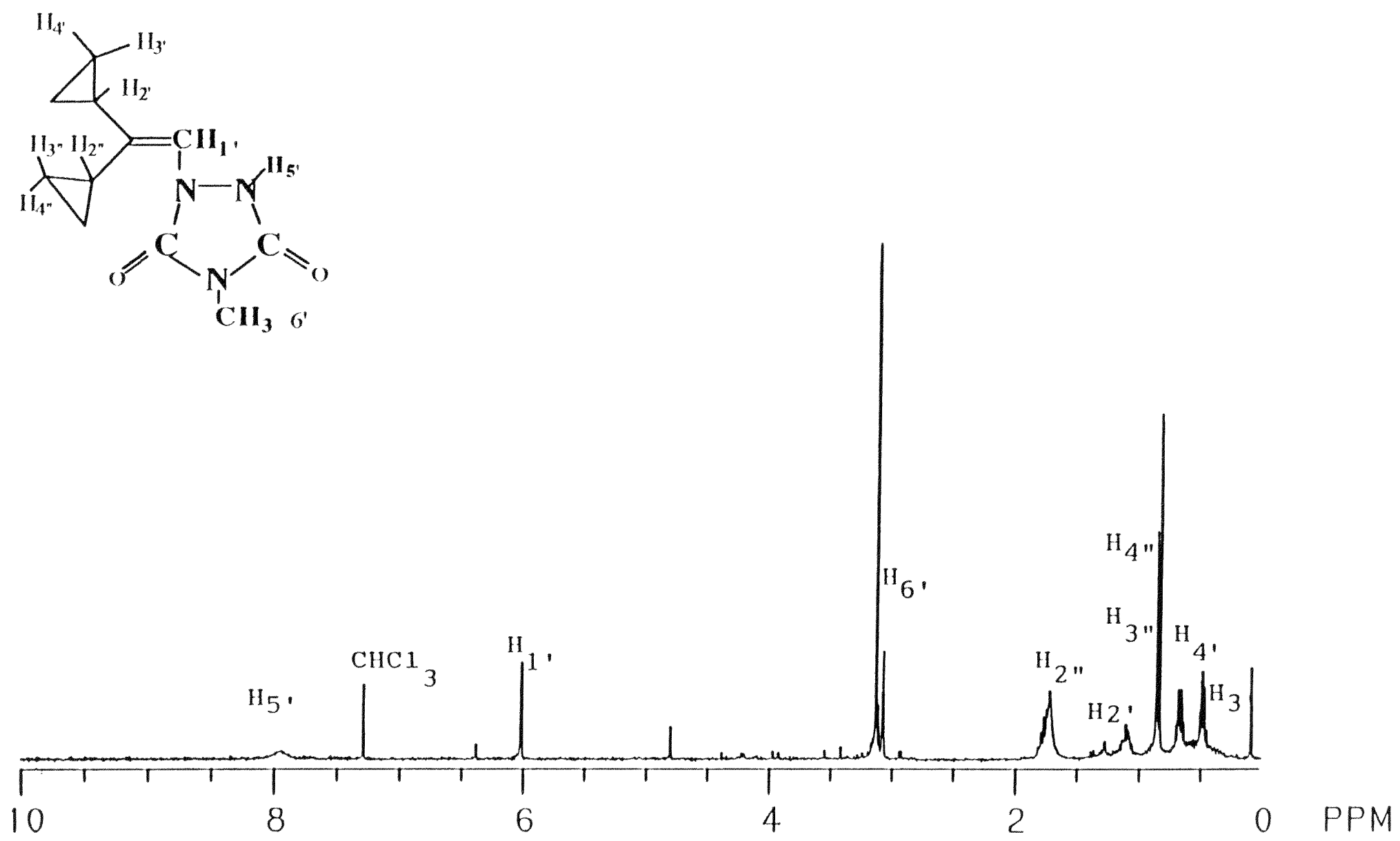
FIGURE XXXII: MASS SPECTRUM OF ENE PRODUCT OF REACTION OF 1,1DICYCLOPROPYLETHYLENE WITH MTAD

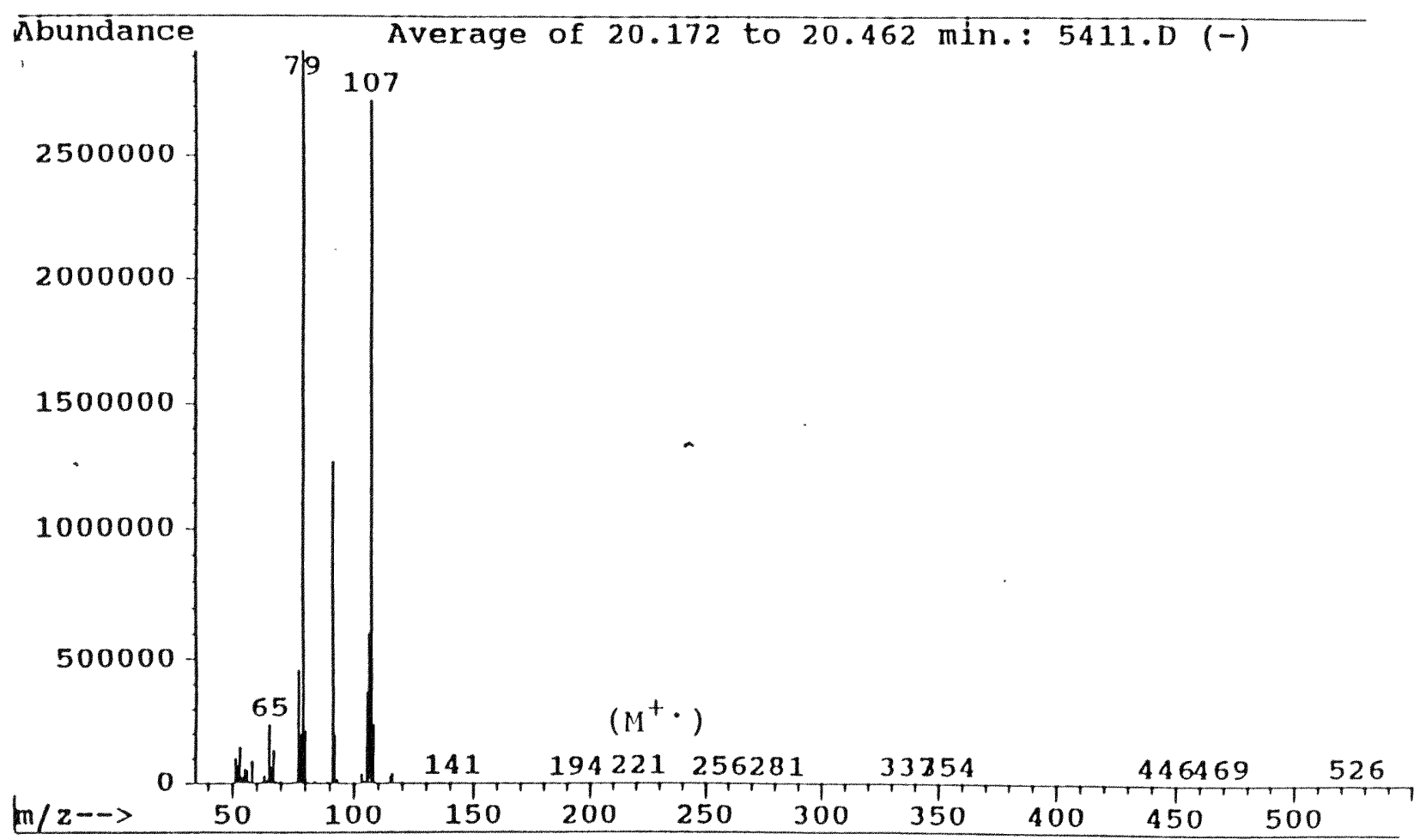


In an attempt to detcrmine if the diazctidine will convert into the ene product at higher temperatures, the $2+2$ product was heated for 5 hours in refluxing benzene and in refluxing xylene. The diazetidine product was found to be quite stable and did not undergo rearrangement at either temperature.

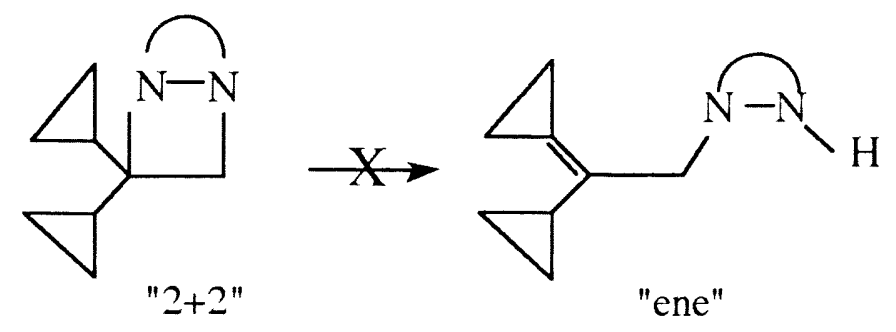

SOLVENT TRAPPING STUDIES:

For the solvent trapping study, an equimolar amount of HPLCgrade methanol was added immediately to an NMR lube containing $20 \mathrm{uL}$ of the olefin followed by the addition of $0.020 \mathrm{~g}$ of MTAD (or MTAD/CDCl3) in $1 \mathrm{~mL}$ of $\mathrm{CDCl}_{3}$. Methanol was used in equimolar amounts with respect to the olefin and MTAD because a side reaction between methanol and MTAD was observed in presence of larger amounts of methanol. The mixture was inverted several times to ensure proper mixing. The solution was kept in an Dewar with icewater. The initial red color of the MTAD dissappeared after several minutes indicating that the reaction was complete. ${ }^{1} \mathrm{H}-,{ }^{3} \mathrm{C}-\mathrm{NMR}$ and the Attached Proton Test (APT) were used to identify and characterize the reaction product. 
Gas chromatographic analysis and NMR spectroscopy showed that a methanol adduct was obtaincd in $-100 \%$ yicld. No formation of $2+2$ or ene product was detected when the reaction is run in the presence of methanol.

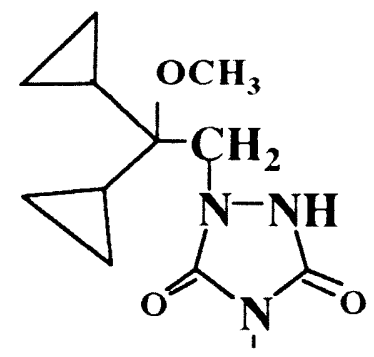

$\mathrm{CH}_{3}$

Markonikov's adduct

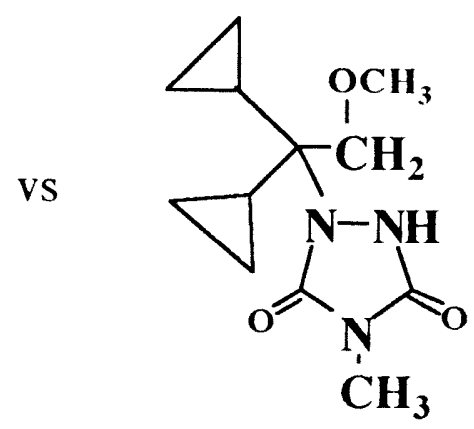

anti-Markonikov's adduct

Although spectral evidence suggests a single product is formed in the presence of methanol. ${ }^{1} \mathrm{H}-\mathrm{NMR}$ are expected to be similar for Markonikov or anti-Markonikov addition, $13{ }^{3} \mathrm{C}-\mathrm{NMR}$ and APT spectrum demonstrate that the methoxy group is attached to a quaternary carbon buried under the solvent $\mathrm{CDCl}_{3}$. We thus determine methanol adds to the more substituted carbon (Markonikov's addition). The presence of geminal cyclopropyl groups in 1,1-dicyclopropylethylene increases the positive charge at that carbon; formation of the Markonikov product suggests the presence of either an AZI (closed zwitterion) or the open zwitterion. Final characterization was achieved by $1^{1} \mathrm{H}_{-},{ }^{3}{ }^{3} \mathrm{C}-\mathrm{NMR}$ and the Attached Proton Test (Figures XXXIII-XXXV). 
${ }^{1} \mathrm{H}-\mathrm{NMR}\left(300 \mathrm{MHz}, \mathrm{CDCl}_{3}\right) \delta \quad(\mathrm{ppm}): 8.0 \quad(\mathrm{~b}, 1 \mathrm{H}), 3.46 \quad(\mathrm{~s}, 2 \mathrm{H}), 3.3 \quad(\mathrm{~s}$, 3H), $3.0(\mathrm{~s}, 3 \mathrm{H}) .{ }^{13} \mathrm{C}-\mathrm{NMR}\left(300 \mathrm{MH} /, \mathrm{CDCl}_{3}\right) \& \quad(\mathrm{ppm}): 155.8$ (s), 154.8 $(\mathrm{s}), 78.5(\mathrm{~s}), 52.53(\mathrm{t}), 51.76(\mathrm{q}), 26.53(\mathrm{q}), 14.7(\mathrm{~d}), 1.8(\mathrm{t}), 1.6(\mathrm{t})$.

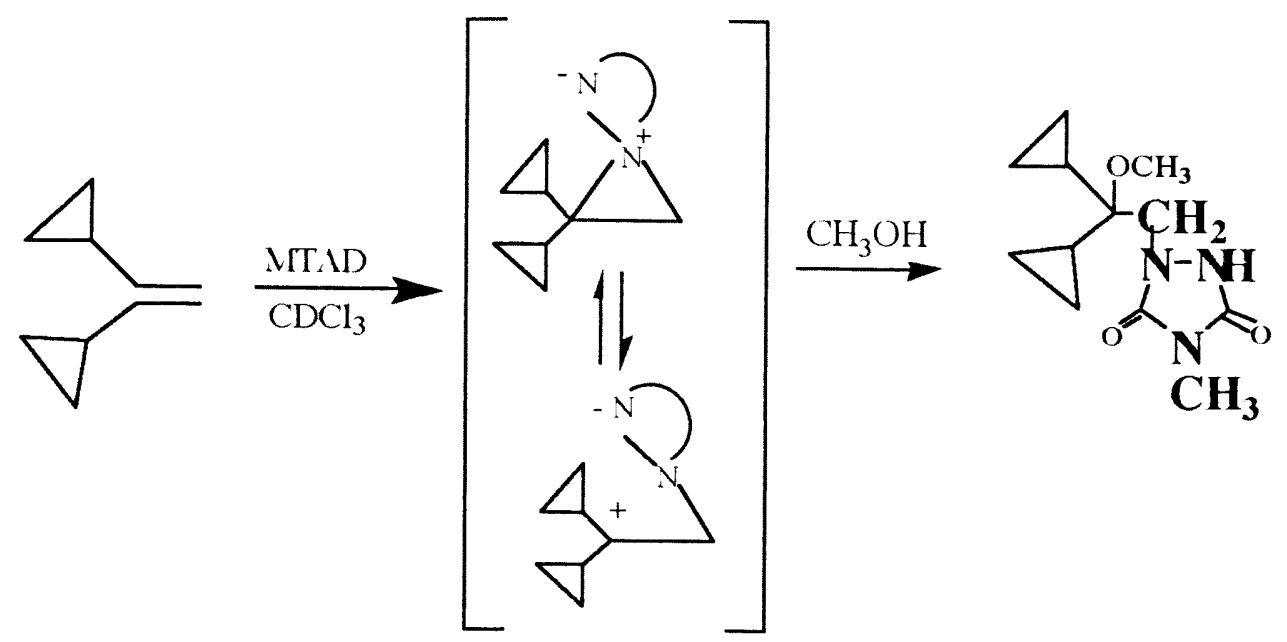

Low temperature experiments were conducted at $-78^{\circ} \mathrm{C}$ in an attempt to observe the zwitterionic intermediate. An NMR tube containing $20 \mathrm{uL}$ of 1,1-dicyclopropylethylene and $0.020 \mathrm{~g}$ of MTAD in $\mathrm{CD}_{2} \mathrm{Cl}_{2}$ was kept in a Dewar flask with dry ice-acetone. This solution was careful transferred to a pre-cooled NMR magnctic avoiding warming of the solution. Unfortunately these variable temperature experiments were hampered by the formation of crystals during the reaction. The solubility problem was aleviated using a more polar solvent $\mathrm{d} 6$-acetone. The rate of reaction at $-78^{\circ} \mathrm{C}$ was extremely slow. Even after several days no appreciable changes was observed in the reaction mixture as determined by ${ }^{1} \mathrm{H}$ NMR. In addition, the formation of an AZI intermediatc is not obscrved at -78 ${ }^{\circ} \mathrm{C}$ and the characteristic red color remains in the reaction mixture. 
FIGURE XXXIII: 1 II NMR SPECTRUM OF REACTION OF 1,1-DICYCLOPROPYLETHYLENE WITH MTAD IN THE PRESENCE OF METHANOL

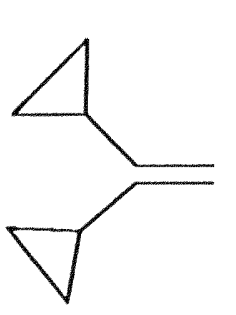<smiles>CN1C(=O)N=NC1=O</smiles>
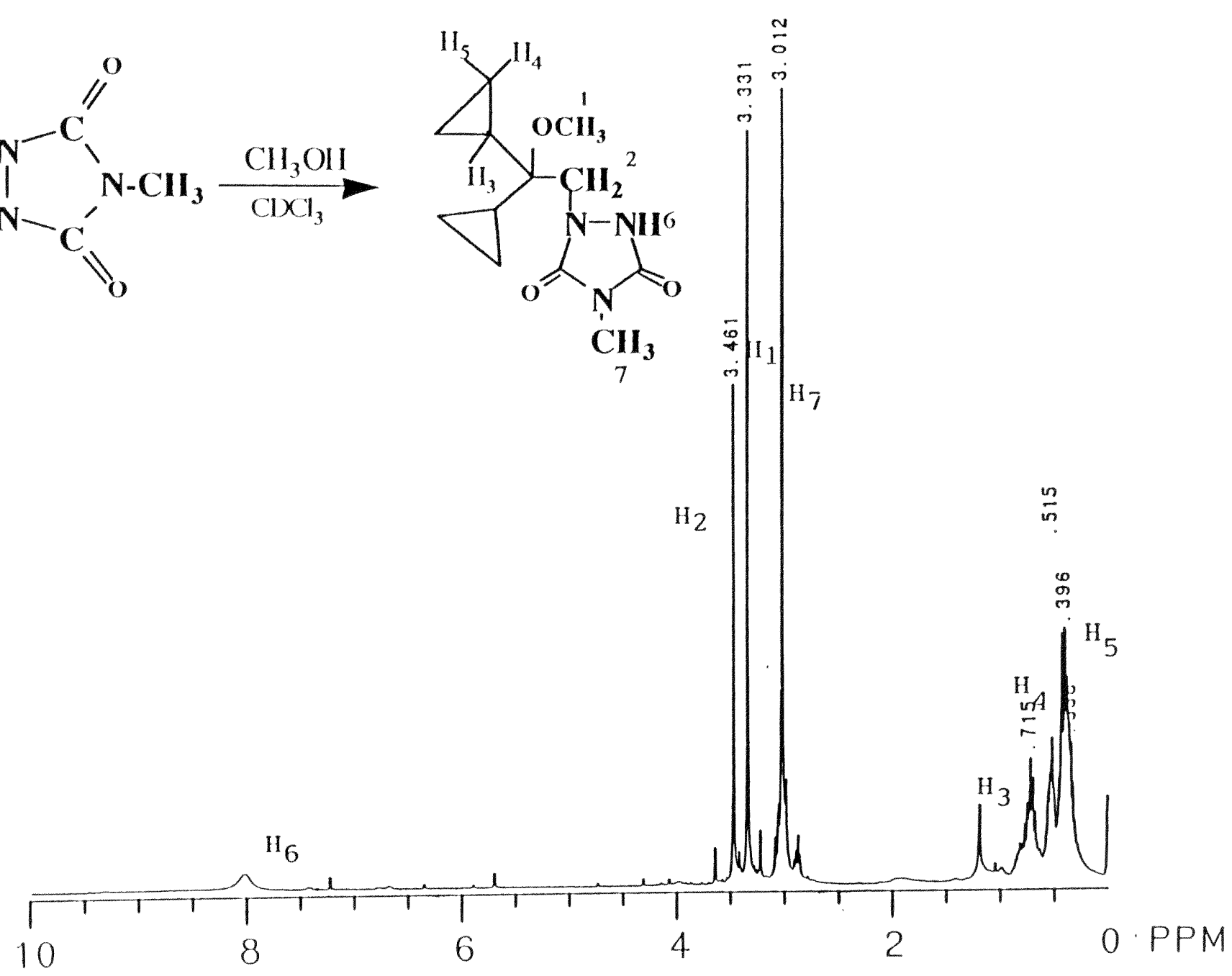
FIGURE XXXIV: 1 3C NMR SPECTRUNI OF REACTION OF 1,1-DICYCLOPROPYLETIIYENE IVITII MTAD IN TIIE PRESENCE OF METIIANOL

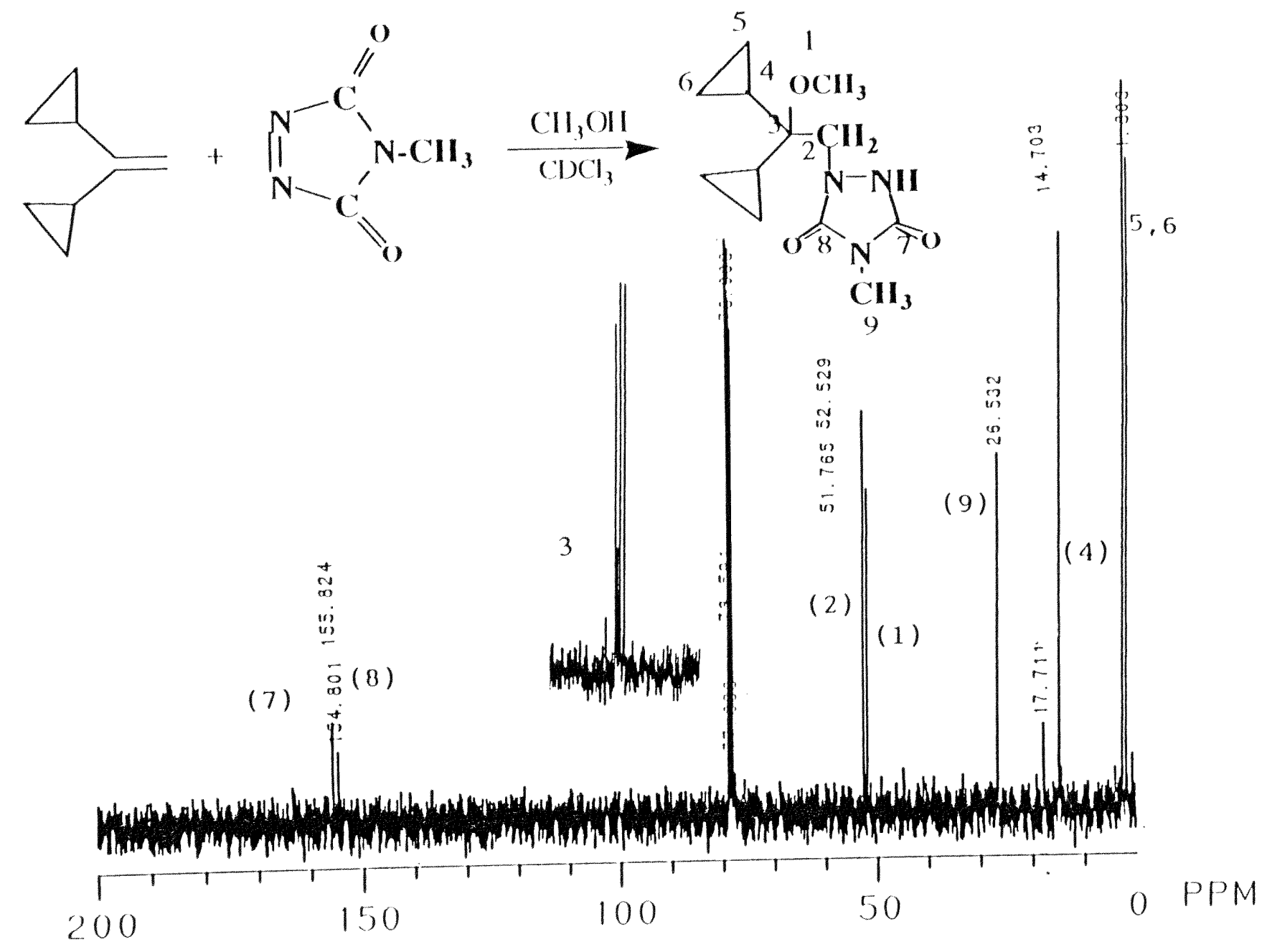


FIGURE XXXV: ATTACHED PROTON TEST SPECTRUM OF REACTION OF 1,1DICYCLOPROPYLETHYLENE WITH MTAD IN THE PRESENCE OF METHANOL

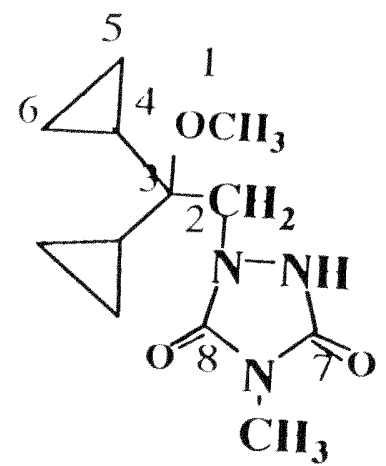

9

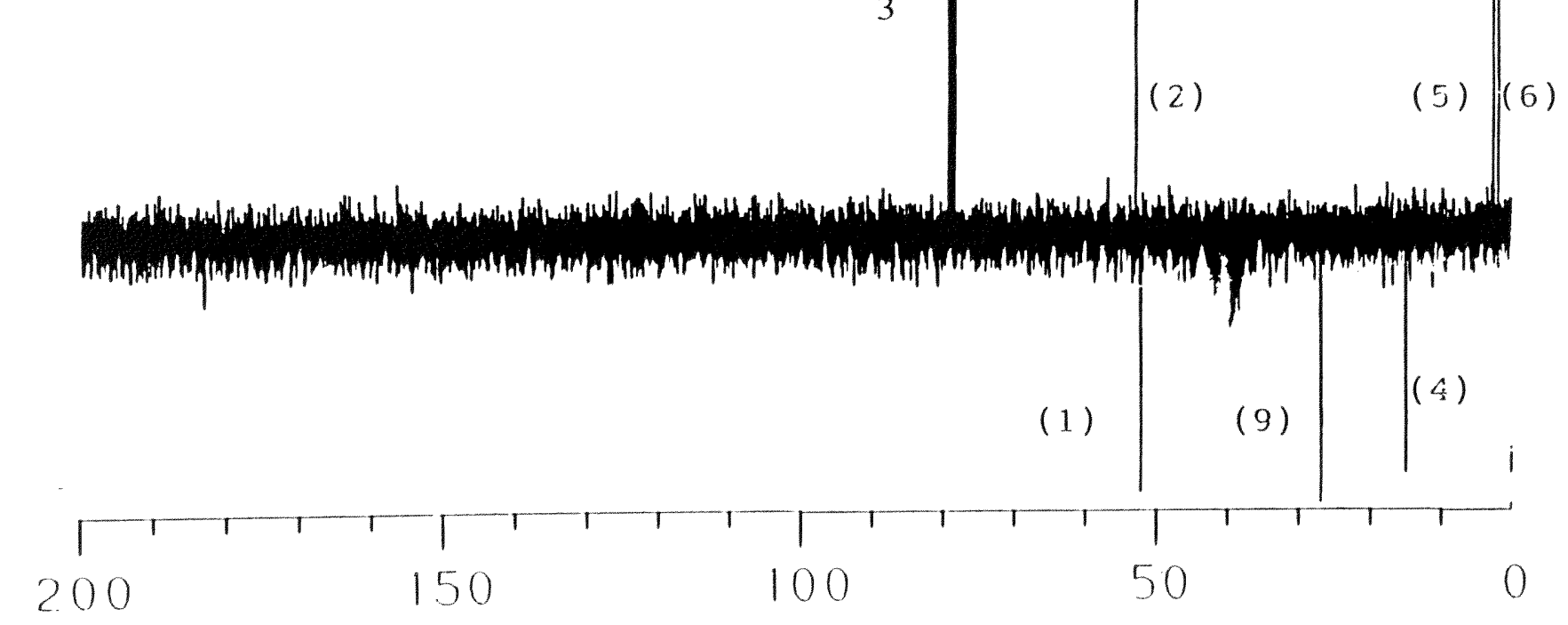




\subsubsection{REACTION OF 4-METHYL-1,2,4-TRIAZOLINE-3,5-DIONE (MTAD) WITH CIS-1,2-DICYCLOPROPYLETHYLENE}

cis-1,2-Dicyclopropylethylene was used to probe the stereochemical consequences of the reaction of olefins with RTAD. The reaction of cis-1,2-dicyclopropylethylene is expected to yicld the $2+2$ and ene products. Trapping experiments will provide cvidence for the involvement of a zwittcrionic intermediate. The stereochemistry of the $2+2$ and methanol adducts will provide insight regarding the nature of the possible intermediates.

\section{RESULTS:}

Approximately $20 \mathrm{uL}$ of a pure olcfin (cis-1,2dicyclopropylethylene) collected by prep-GC was mixed with $1 \mathrm{~mL}$ of $\mathrm{CDCl}_{3}$ in an NMR tube kept at $0{ }^{\circ} \mathrm{C}$ with ice-water in a Dewar flask. An equimolar amount of MTAD (from Aldrich Co.) or a solution of MTAD in $\mathrm{CDCl}_{3}$ was added to the alkene solution. Upon addition of $\mathrm{MTAD} / \mathrm{CDCl}_{3}$ to the alkene, and after $5 \mathrm{~min}$ the red color fades away indicating that the reaction was complete. ${ }^{1} \mathrm{H}-,{ }^{3} \mathrm{C}-\mathrm{NMR}$ and the Attached Proton Test (APT) were used to identify and characterize the reaction products.

Gas chromatography and NMR spectroscopy was used to monitor the disappearance of the reactants. ${ }^{1} \mathrm{H}-,{ }^{1}{ }^{3} \mathrm{C}-\mathrm{NMR}$ and the Attached Proton Test were used to identify and characterize the reaction products. cis-Dicyclopropylethylene and MTAD yield only 
the cycloaddition product, a diazctidinc product, in which cis strereochemistry is maintained (Figures XXXVI-XXXVIII).

${ }^{1} \mathrm{H}-\mathrm{NMR}(300 \mathrm{MH}, \mathrm{CDCl} 3)$ ) $\delta$ (ppm): 3.9 (dd, 2H), 3.1 (s, 3H), 1.4 $(\mathrm{m}, \quad 2 \mathrm{H}), \quad 0.7(\mathrm{~m}, 4 \mathrm{H}), 0.25(\mathrm{~m}, 4 \mathrm{H}), \quad{ }^{3} \mathrm{C}-\mathrm{NMR}\left(300 \mathrm{MHz}, \mathrm{CDCl}_{3}\right)$ \& $(\mathrm{ppm}): 165.6(\mathrm{~s}), 72.6(\mathrm{~d}), 27.41(\mathrm{q}), 11.64(\mathrm{~d}), 4.6(\mathrm{l}), 4.0(\mathrm{t})$.

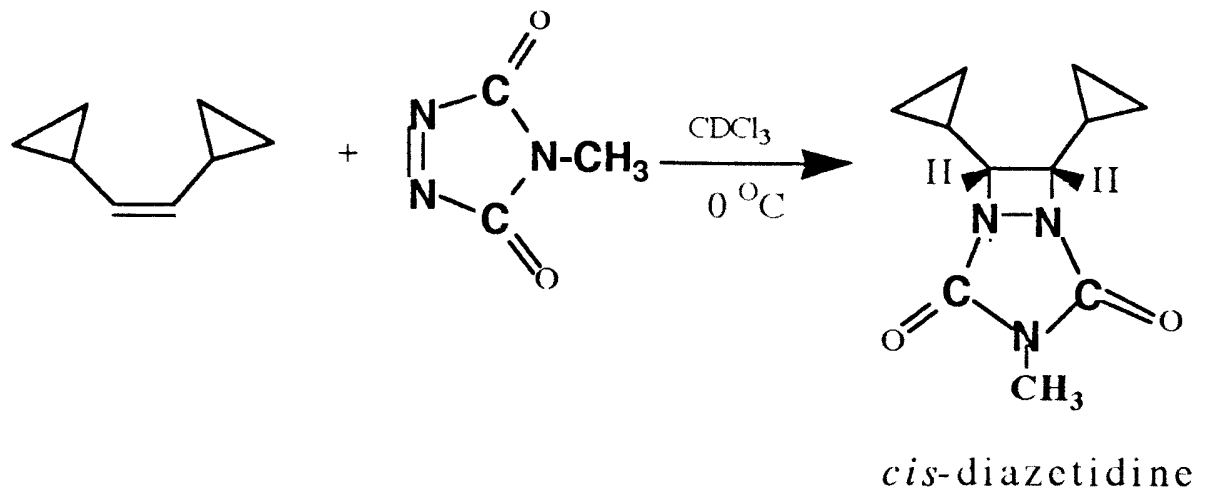

These results indicate the reaction proceds via a concerted mechanism or a closed zwitterion, aziridinium imide, since the $2+2$ addition occurs stereospecifically. The product cis-diazetidine shows a coupling constant (J) (Figure XXXIX) similar to the cis coupling constant observed by Jensen's ${ }^{15}\left[\mathrm{~J}_{\mathrm{cis}}=5.6-6.8 \mathrm{~Hz}\right.$ and $\mathrm{J}_{\mathrm{trans}}=3.7-4.6$ $\mathrm{Hz}]$. 
FIGURE XXXVI: 1 II NMR SPECTRUM OF REACTION OF CIS-DICYCLOPROPYLETHYLENE WITH MTAD

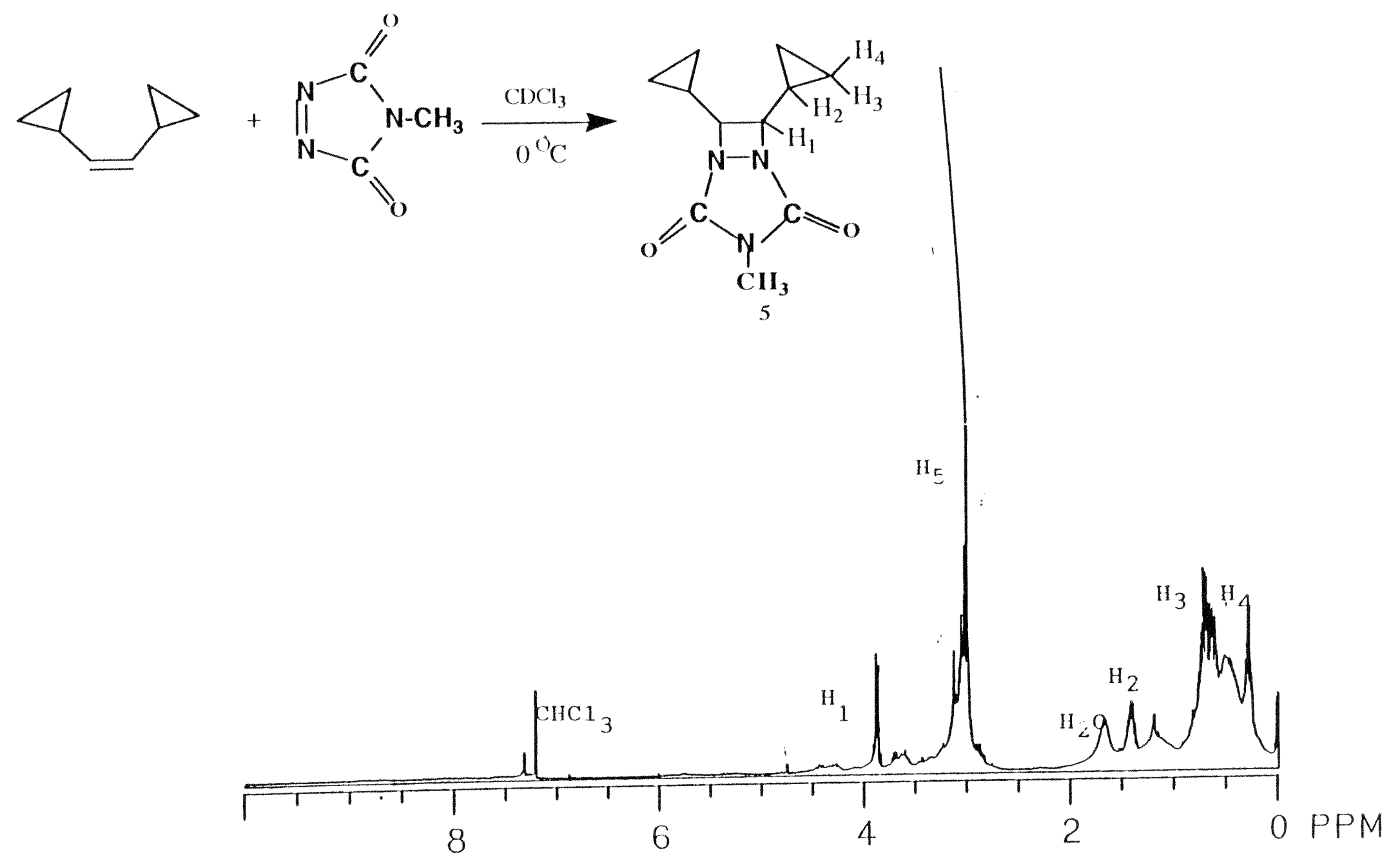


FIGURE XXXVII: 13 C NMIR SPECTRUM OF REACTION OF CIS-DICYCLOPROPYLETIYLENE WITII MTAD
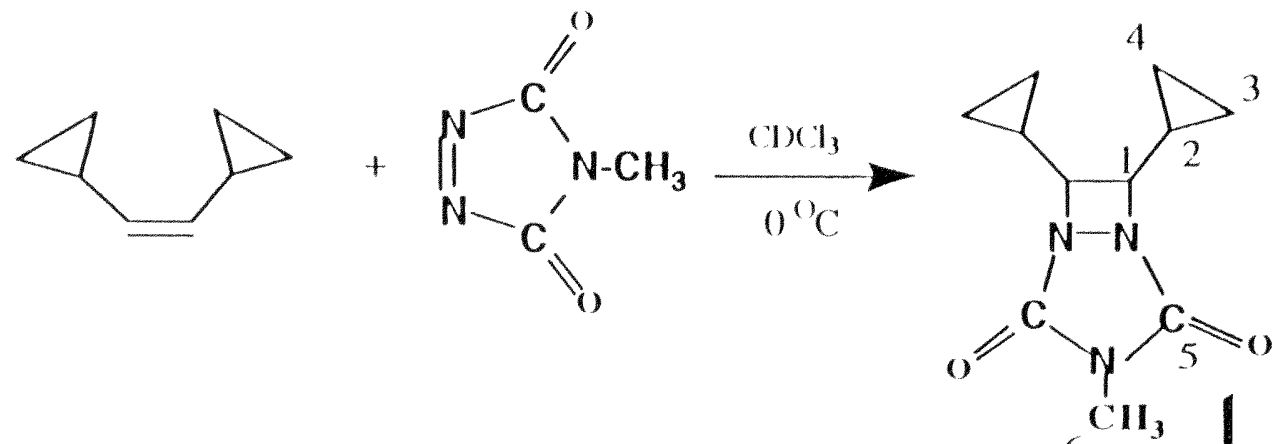

(1)

(5)

曾

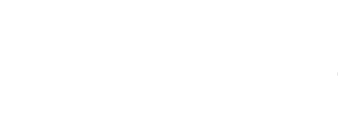

2
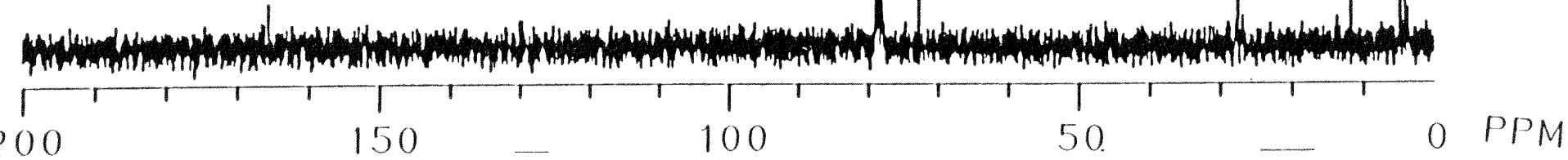
FIGURE XXXVIII: ATTACHED PROTON TEST SPECTRUM OF REACTION OF CISDICYCLOPROPYLETHYLENE WITH MTAD
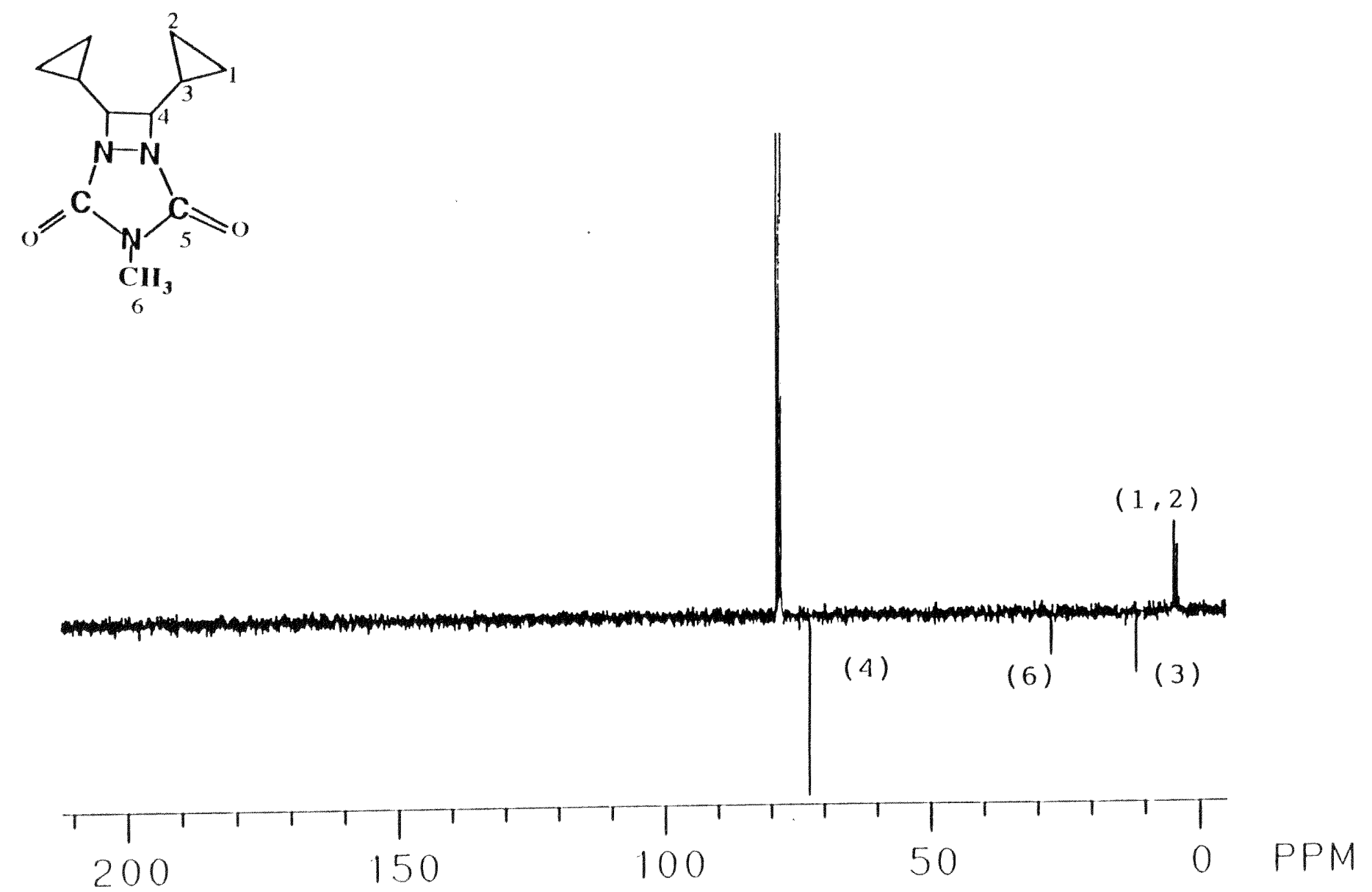
FIGURE XXXIX: COUPLING CONSTANT OF DIAZETIDINE OF REACTION OF CIS. DICYCLOPROPYLETHYLENE WITH MTAD
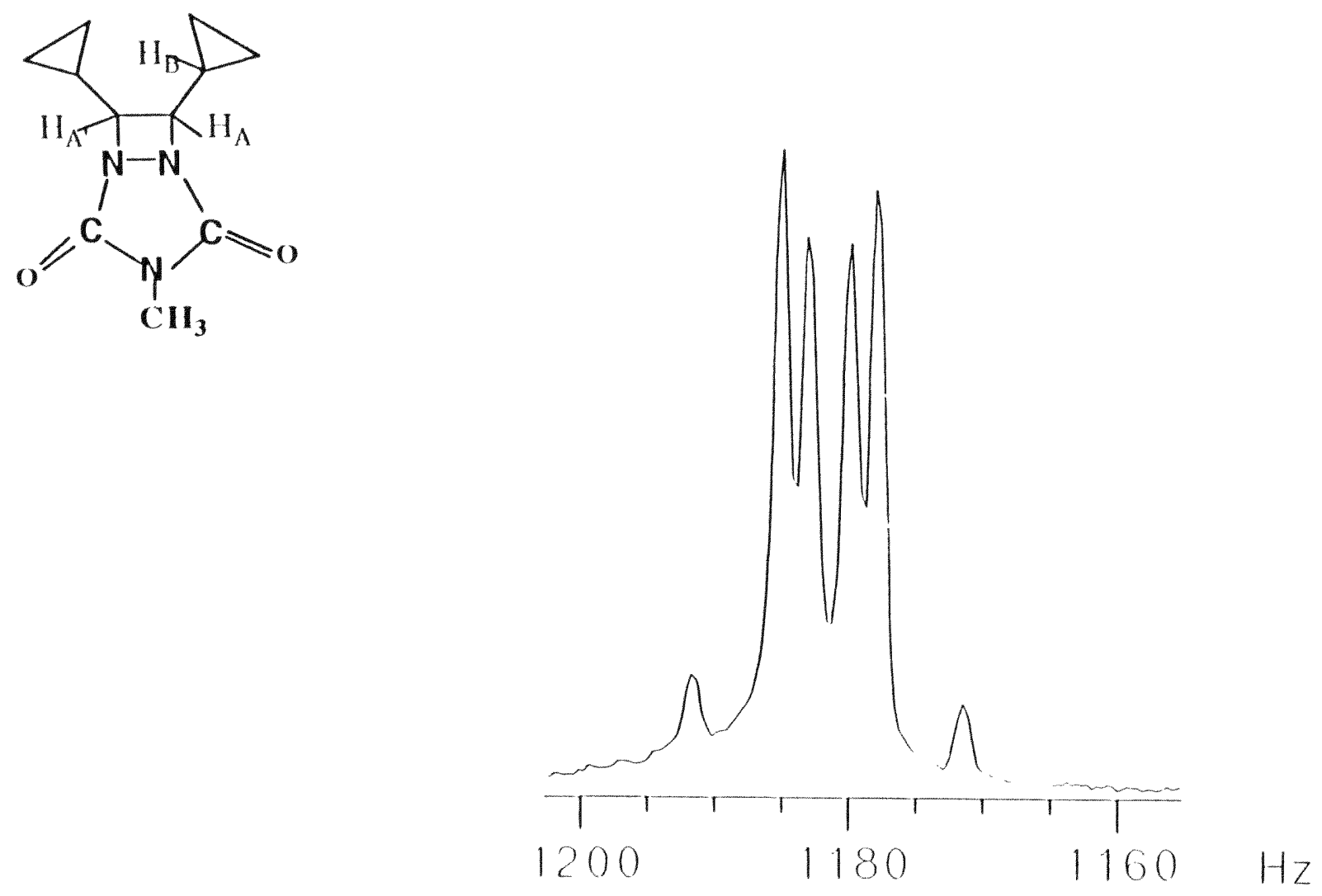


\section{SOLVENT TRAPPING STUDY:}

When the reaction of cis-dicyclopropylethylene with MTAD is carried out in the presence of methanol a new product is observed in addition to the $2+2$ product. The new product is observed in a 1:1.5 ratio as determined by gas chromatographic analysis and NMR spectroscopy ${ }^{1} \mathrm{H}-, 13 \mathrm{C}-\mathrm{NMR}$ and the Attached Proton Test (Figures XL-XLII). The two products were separated by column chromatography on silica gel (200-340 mesh, 5 in length, $\sim 1.5$ in $\mathrm{i}$. d.), using a 2:1 ethyl acetate/hexane as eluant. Characterization of the

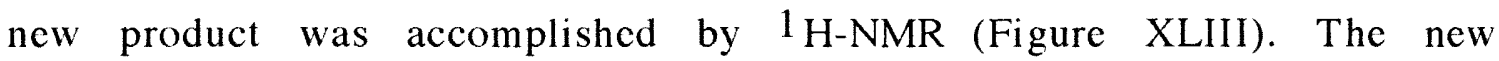
product is a methanol adduct ${ }^{1} \mathrm{H}-\mathrm{NMR}\left(300 \mathrm{MHz}, \mathrm{CDCl}_{3}\right) \delta(\mathrm{ppm}): 7.6$ $(\mathrm{b}, 1 \mathrm{H}), 3.5(\mathrm{~d}, 3 \mathrm{H}), 3.4(\mathrm{dd}, 1 \mathrm{H}), 3.1(\mathrm{~s}, 3 \mathrm{H}), 2.7(\mathrm{dd}, 1 \mathrm{H})$.

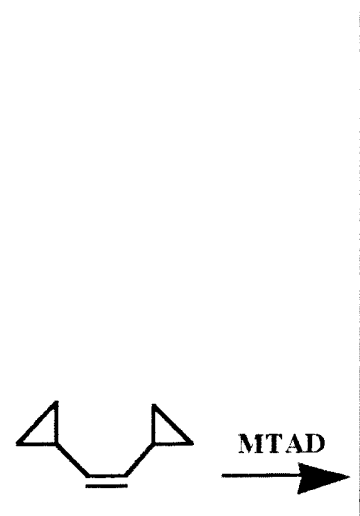

AZIRIDINIUM IMIDE
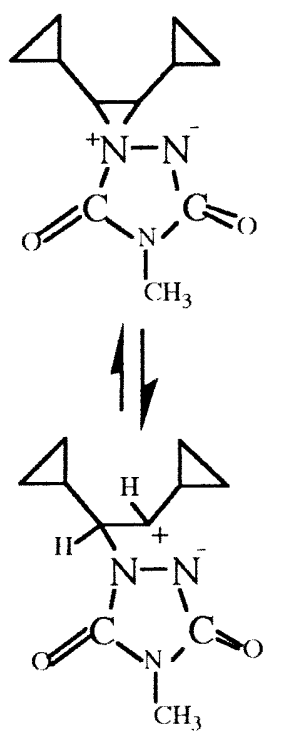

OPEN ZI
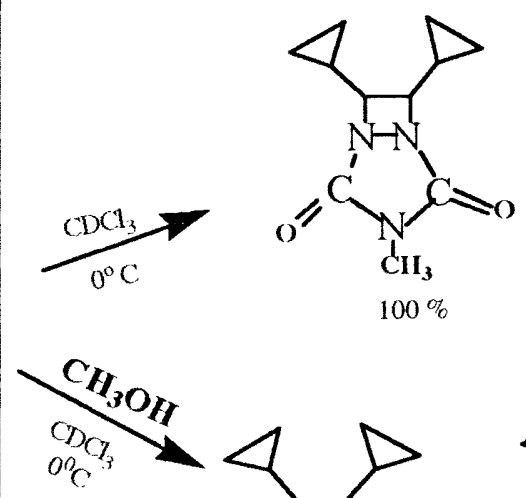

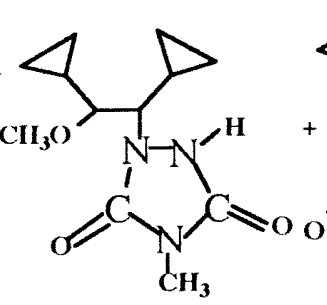

$75 \%$<smiles>Cn1c(=O)n(C2CC2)n(C2CC2)c1=O</smiles>

$25 \%$ 
The formation of the $2+2$ product and methanol adducts suggest the involvement of a zwitterion which rearranges to the $2+2$ product in competitition with nucleophilic attack by methanol.

If an open zwitterion is involved and bond rotations occur addition of methanol is expected to yicld four stereoisomers $(R, R)$, $(S, S),(R, S),(S, R)$, while the involvement of the closed zwittcrion (AZI) will lead to enantiomeric pairs $(R, S) \&(S, R)$ upon addition as shown below.
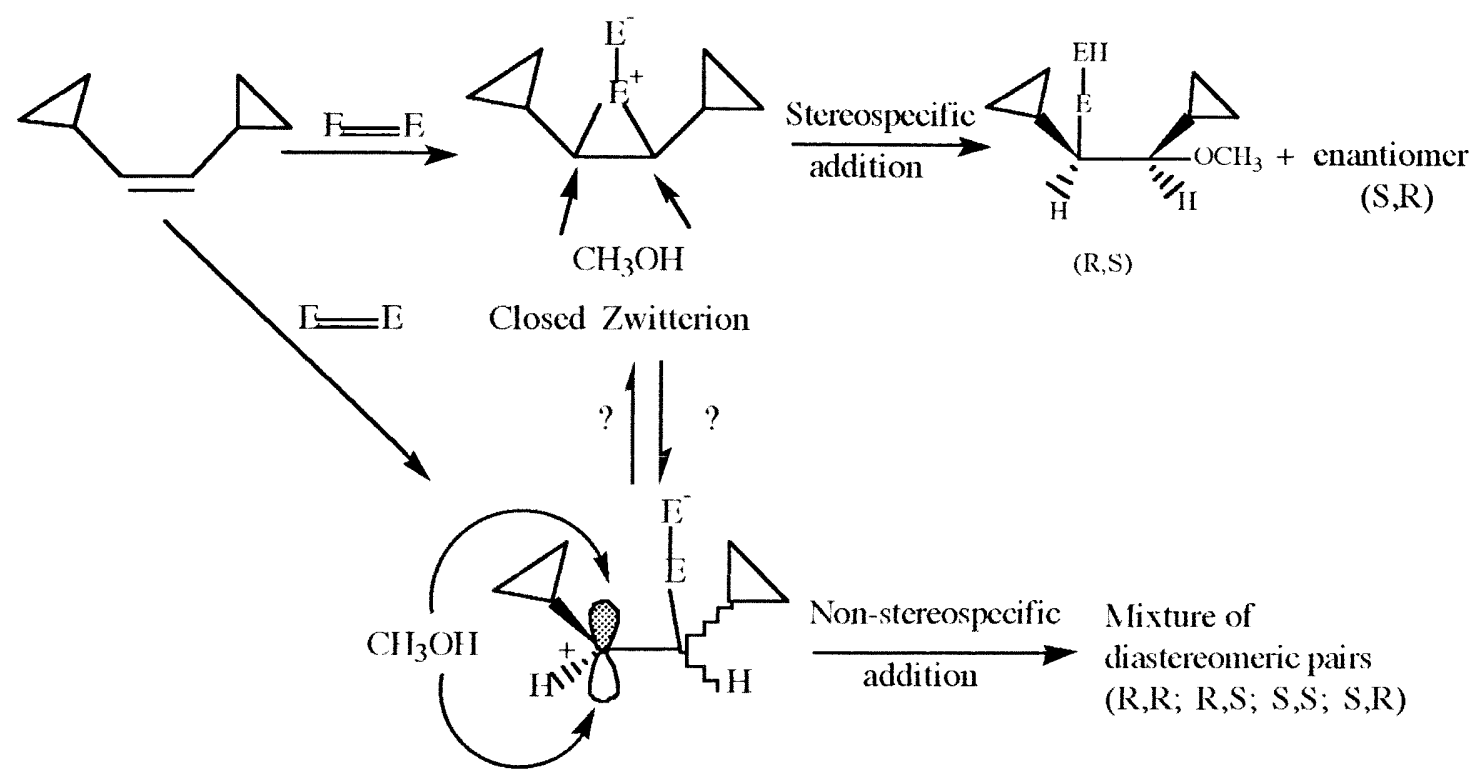

Open Zwitterion

The presence of an aziridinium imide (AZI) as the intermediate is supported when the isolated methanol adduct is mixed with a chiral reagent $\mathrm{Eu}(\mathrm{hcf}) 3$ and the mixture is characterized by NMR spectroscopy. ${ }^{1}$ H-NMR spectrum shows no major difference in the presence of the chiral reagent. Our result demonstrated the presence of one pair of enantiomers that will support the implication of an aziridinium imide as the intermediate (Figure XLIV). 
FIGURE XL: 1 II NMR SPECTRUM OF REACTION OF $c$ is -DICYCLOPROPYLETIYLENE IVITI MTAD IN THE PRESENCE OF METHANOL

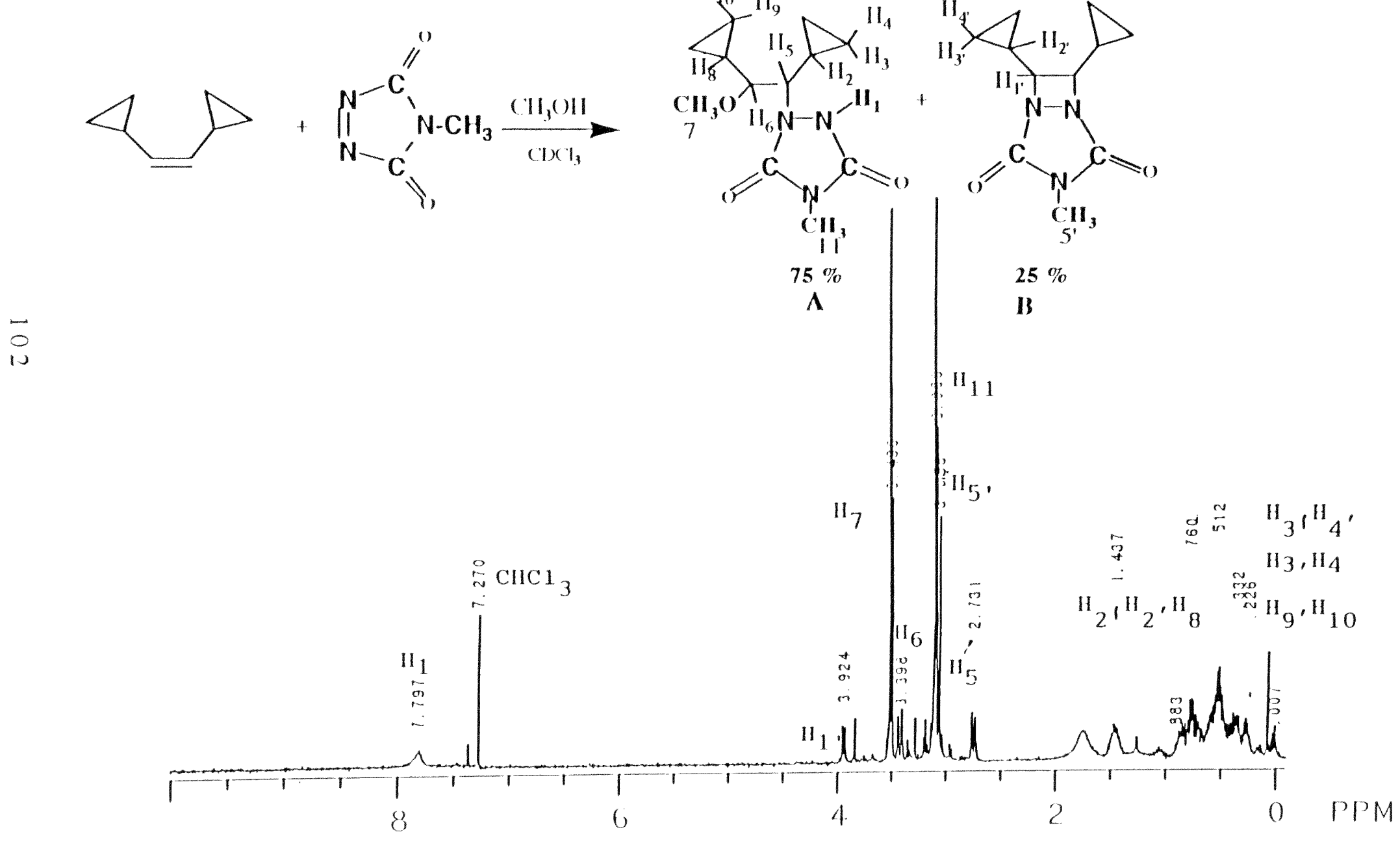


FIGURE XLI: $13^{3}$ C NMR SPECTRUM OF REACTION OF $c$ is-DICYCLOPROPYLETHYLENE WITH MTAD IN THE PRESENCE OF METHANOL
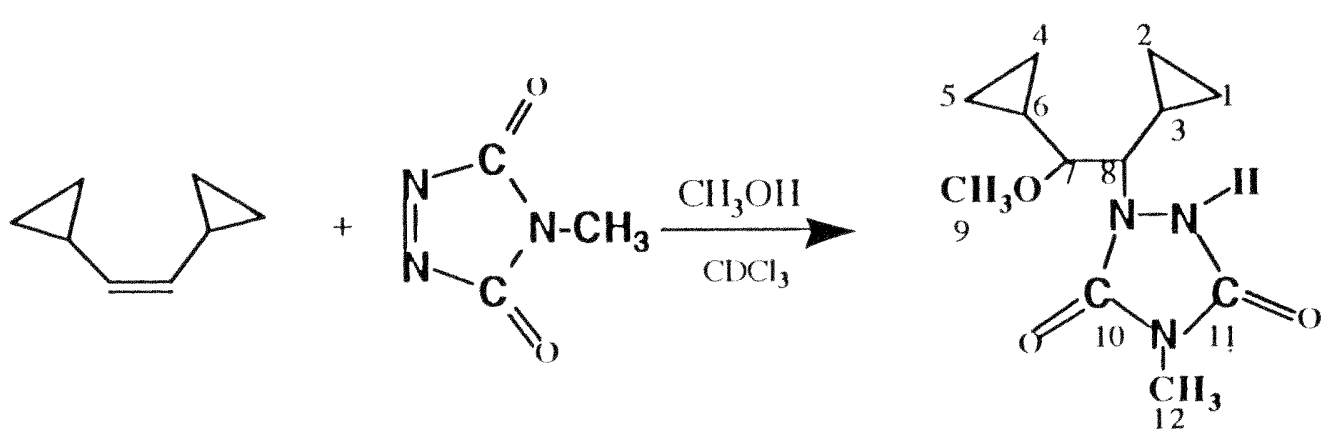

$75 \%$

A

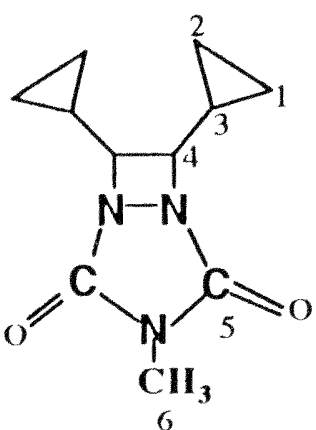

$25 \%$

$$
5^{\cdot}, 10,11
$$

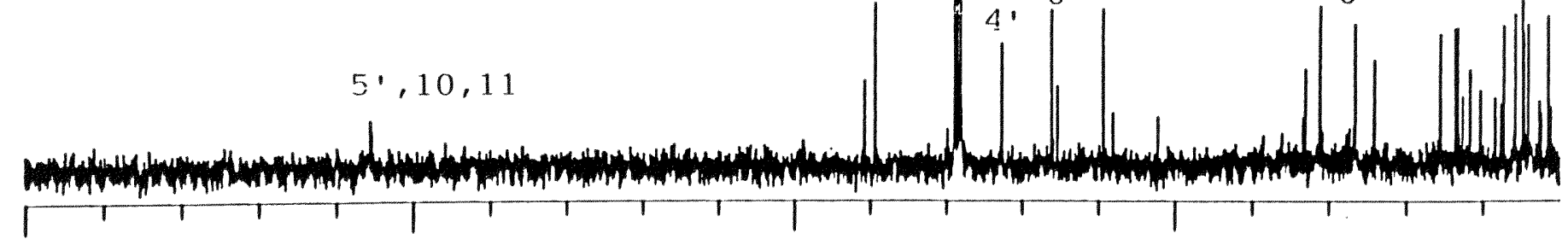

200

150

100

50

$0 \quad P P M$ 
FIGURE XLII: ATTACHED PROTON TEST SPECTRUM OF REACTION OF $c$ is DICYCLOPROPYLETHYLENE WITH MTAD IN THE PRESENCE OF METHANOL
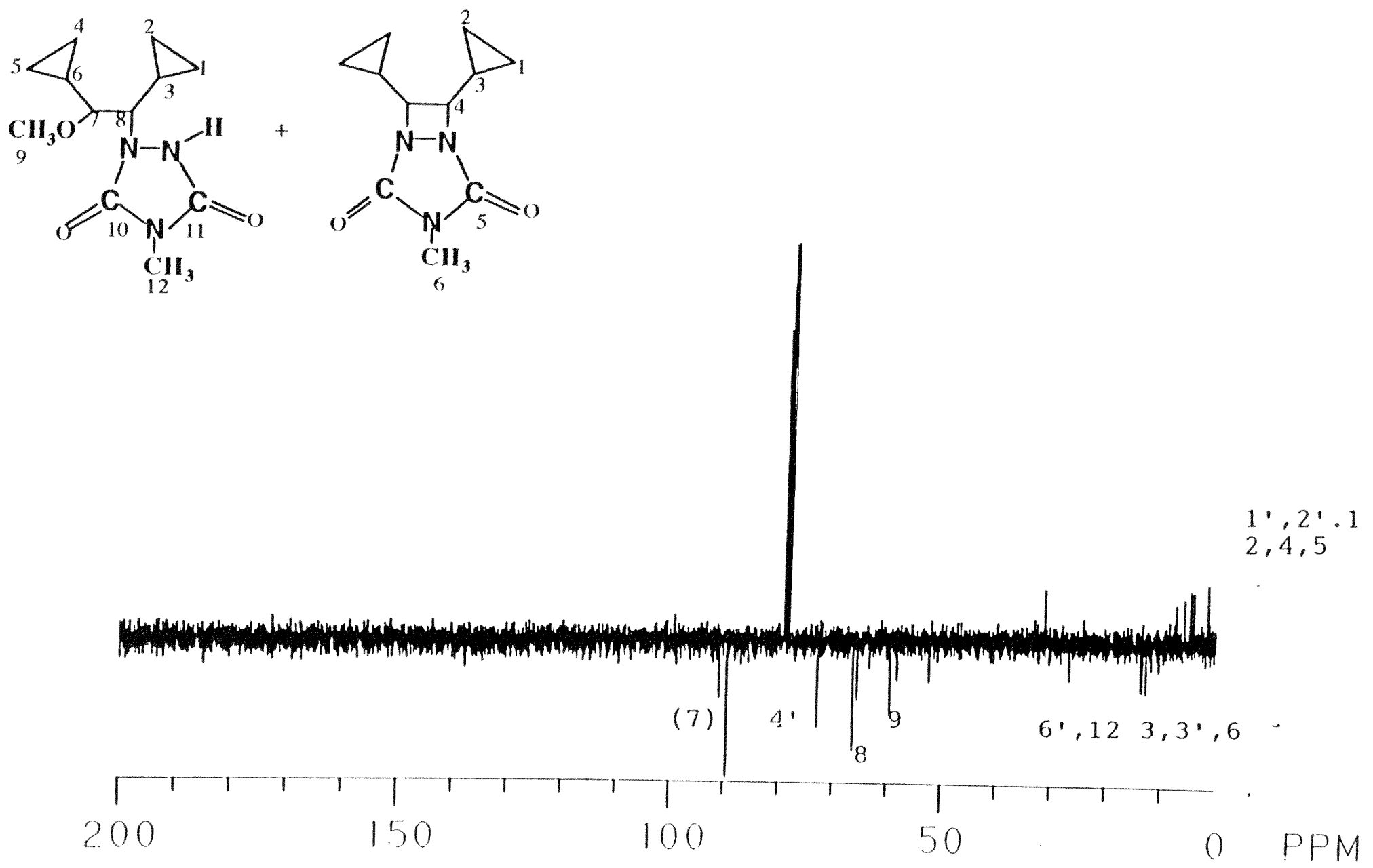
FIGURE XLIII: 1 H NMR SPECTRUM OF METHANOL ADDUCT OF REACTION OF $c$ isDICYCLOPROPYLETHYLENE WITH MTAD
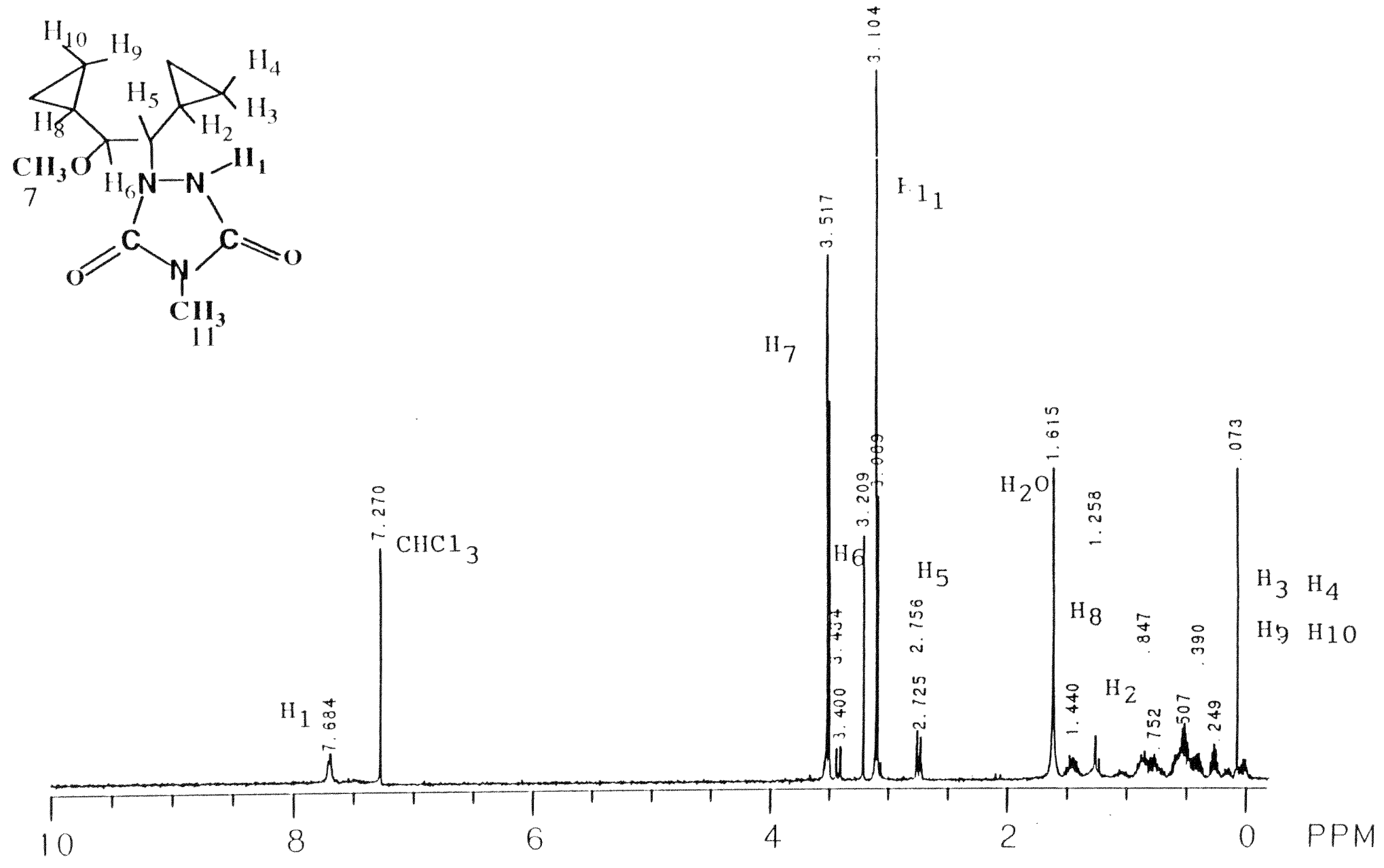
FIGURE XLIV: 1 H NMR SPECTRUM OF METHANOL ADDUCT OF REACTION OF $c$ isDICYCLOPROPYLETHYLENE WITH MTAD IN THE PRESENCE OF CHIRAL REAGENT<smiles>COC(C1CC1)n1c(=O)n(C)c(=O)n1C</smiles>

(R,S), (S,R)

$\vec{\circ}$

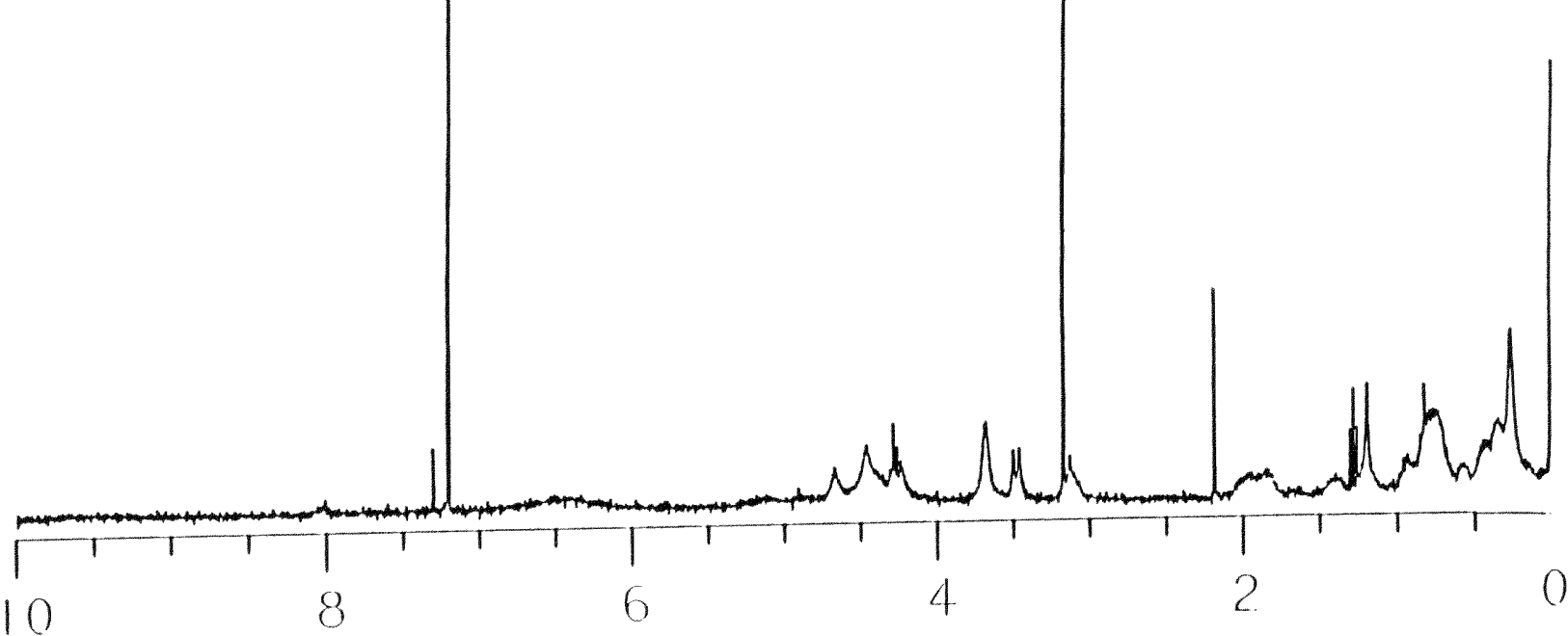




\subsubsection{REACTION OF 4-METHYL-1,2,4-TRIAZOLINE-3,5-DIONE (MTAD) WITH $\operatorname{trans-1,2-DICYCLOPROPYLETHYLENE~}$}

$\operatorname{trans-1,2-Dicyclopropylethylene}$ is expected to react in a fashion similar to cis-1,2-dicyclopropylethylene giving the 2+2 cycloaddition product. The stereochemistry of the diazetidine is expected to be retained if the intermediate resembles an AZI. If the intermediate is an open zwitterion mixed stereochemistry should be observed. Methanol adducts are expected to be formed supporting the intermediacy of a zwitterionic species.

\section{RESULTS:}

Approximately $10 \mathrm{uL}$ of a pure olefin (trans-1,2dicyclopropylethylene) collected by prep-GC were mixed with $1 \mathrm{~mL}$ of $\mathrm{CDCl}_{3}$ in an NMR tube kept at $0{ }^{\circ} \mathrm{C}$ with ice-water in a Dewar. An equimolar amount of MTAD (from Aldrich Co.) or a solution of MTAD in $\mathrm{CDCl}_{3}$ was added to the alkene solution. Upon addition of $\mathrm{MTAD} / \mathrm{CDCl}_{3}$ to the alkene, and after $5 \mathrm{~min}$ the red color fades away indicating that the reaction was complete. ${ }^{1} \mathrm{H}-\mathrm{NMR}$ was used to identify and characterize the reaction products. trans-1,2Dicyclopropyl ethylene reacts with $\mathrm{MTAD}$ in $\mathrm{CDCl}_{3}$ affording a cycloaddition product.

Since only small quatities of trans-1,2-dicyclopropylethylene were available, characterization by NMR spectroscopy was not feasible. Despite several attempts to synthesize and purify trans-1,2- 
dicyclopropylethylene from the cis isomer, a mixture containing $15 \%$ of the cis isomer was obtained. This reaction is expected to occur stereospecifically as observed in cis-dicyclopropylethylene and MTAD. Although our efforts, determination of the structure was not possible.

\subsubsection{REACTION OF 4-PHENYL-1,2,4-TRIAZOLINE-3,5-DIONE (PTAD) WITH 1,1-DIISOPROPYLETHYLENE}

1,1-Diisopropylethylene was used as a probe to evaluate the steric factors affecting reactions of 1,1 -disubstituted olefins with RTAD. The expected products are $2+2$ and ene type. The ene product is much less strained than the corresponding ene product from the reaction of 1,1-dicyclopropylethylene and RTAD. The outcome of this reaction provides insight into the steric or electronic, controlling the reaction pathways which affects the product distribution of reaction between 1,1-diisopropylethylene and RTAD.

\section{RESULTS:}

Approximately $10 \mathrm{uL}$ of a pure olefin (1,1-diisopropylethylene) collected by prep-GC were mixed with $1 \mathrm{~mL}$ of $\mathrm{CDCl}_{3}$ in an NMR tube kept at $0{ }^{\circ} \mathrm{C}$ with ice-water in a Dewar flask. An equimolar amount of PTAD (from Aldrich Co.) or a solution of PTAD in $\mathrm{CDCl}_{3}$ was added to the alkene solution. After addition of $\mathrm{PTAD} / \mathrm{CDCl}_{3}$ to the alkene, the reaction was monitored by gas chromatography, and NMR 
spectroscopy. Surprisingly, the red color associated with PTAD persisted for the first 8 hours at room temperature, indicating no or relatively slow reaction. After 24 hours a colorless solution indicates the reaction was complete. The results showed several small peaks from decomposition of PTAD. The reaction of 1,1-diisopropylethylene with PTAD was negligible although presence of several peaks indicate a decomposition reaction of PTAD.

Reaction of 1,1-diisopropylethylene and $\mathrm{RTAD}$ in $\mathrm{CDCl}_{3}$ reacts very slowly. A more polar solvent may promote the reaction by stabilizing the charged or polarized intermediate. Solvent trapping was attempted using $10 \mathrm{uL}$ of methanol. The methanol was added to a mixture containing $10 \mathrm{uL}$ of the pure olefin $(1,1-$ diisopropylethylene) and an equimolar amount of PTAD in an NMR tube. The solution was cooled at $0^{\circ} \mathrm{C}$ with ice-water in a Dewar flask. A very slow reaction was observed which took $\sim 48$ hours to reach completion. Preliminary results suggest the presence of two products characterized by ${ }^{1} \mathrm{H}$ NMR (Figure XLV). Presence of methanol adducts suggest the involvement of a polar intermediate in the reaction pathway, an increase in reactivity is observed as the polarity of the reaction solution is increased indicating the involvement of a polarized or zwitterionic intermediate.

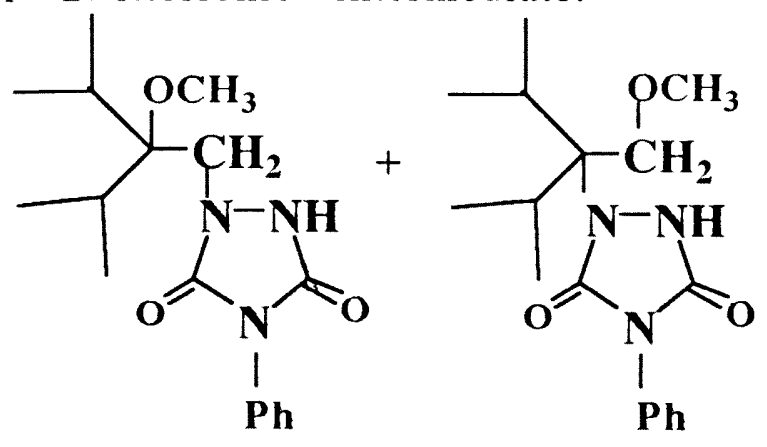


FIGURE XLV: ${ }^{1}$ H NMR SPECTRUM OF REACTION OF 1,1-DIISOPROPYLETHYLENE WITH PTAD IN THE PRESENCE OF METHANOL

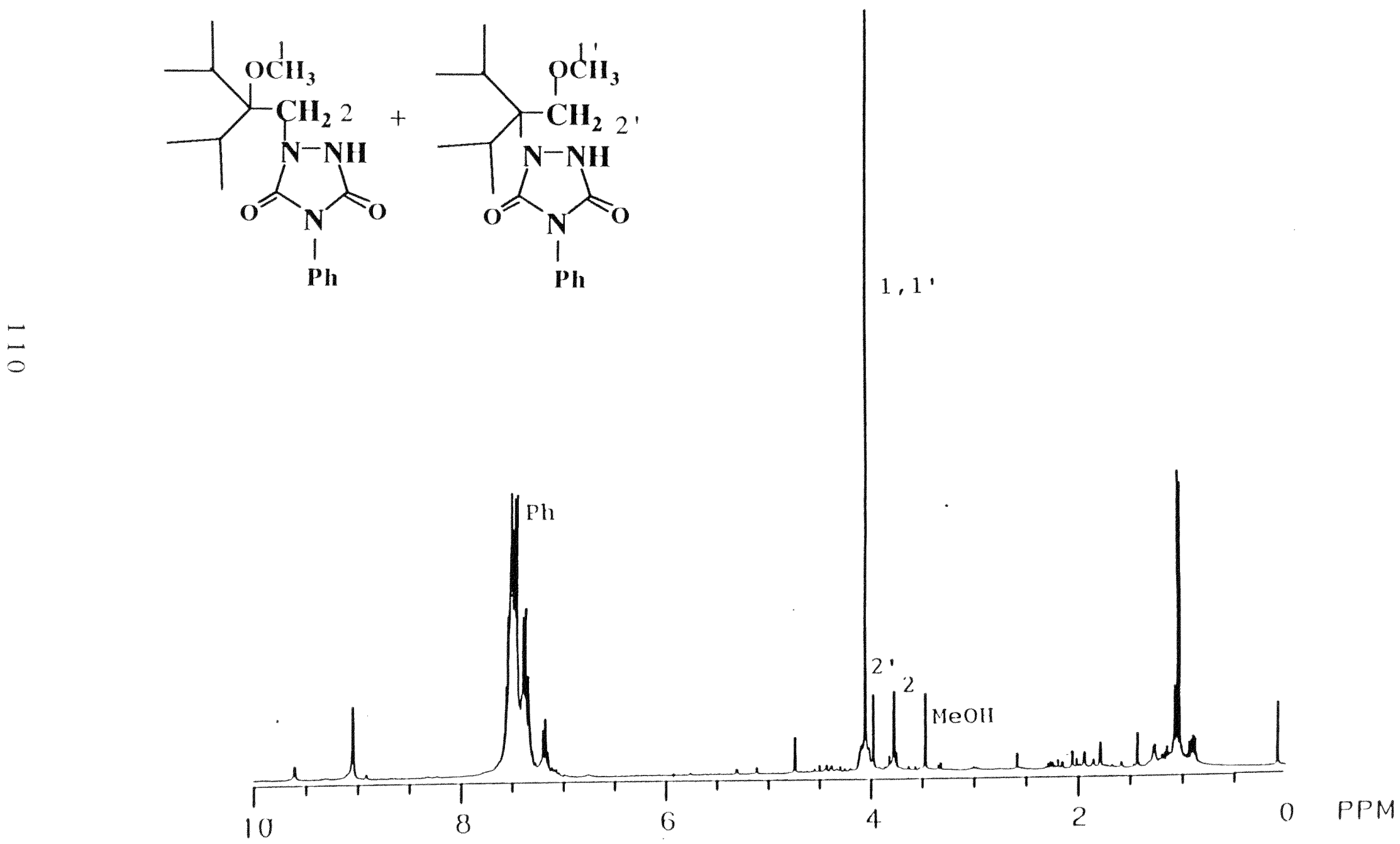

こ 


\subsection{REACTION OF TETRACYANOETHYLENE (TCNE) WITH DICYCLOPROPYL SUBSTITUTED ETHYLENES}

\subsubsection{REACTION OF TETRACYANOETHYLENE (TCNE) WITH 1, 1 -DICYCLOPROPYLETHYLENE}

Nishida ${ }^{30}$ reported the cycloaddition of the substrates $(1,1-$ dicyclopropylehylene, cis-and trans-1,2-dicyclopropylethylene) with tetracyanoethylene (TCNE) gives a $2+2$ product. cisDicyclopropylethylene and trans-dicyclopropylethylene require a polar solvent and elevated temperatures to react with TCNE. This type of cycloaddition takes place between an electron rich olefin with a strong electron demanding reagent as TCNE which is called donoracceptor cycloaddition. They observed the rate of the reaction is fastest for 1,1-dicyclopropylethylene and decreases for the reaction of cis- and trans-dicyclopropylethylene.

The low ionization potential of different cyclopropyethylenes and the correlation between ionization potential in the cycloaddition process of TCNE has been observed. 1,1-Dicyclopropylethylene has a high ionization potential and is the most reactive implying that the presence of the geminal cyclopropyl groups really enhances its reactivity. These studies reveal both the presence of the strongly stabilizing interaction of the cyclopropyl group with an adjacent electron deficient center in the highly polarized transition state as well as the high reactivity of TCNE are the factors that facilitate the cycloaddition. 


\section{GENERAL PROCEDURE:}

Twenty microliters of pure (1,1-dicyclopropylethylene) were mixed with $1 \mathrm{~mL}$ of $\mathrm{CD}_{2} \mathrm{Cl}_{2}$ or acetone, $\mathrm{d}_{6}$ and placed in a NMR tube which was kept in a Dewar llask containing an ice-water bath until the temperature equilibrated. An equimolar amount of TCNE was then added. After mixing the contents properly, a brown color developed and persisted.

\section{RESULTS:}

${ }^{1} \mathrm{H}-, \quad{ }^{1}{ }^{3} \mathrm{C}-\mathrm{NMR}$, Attached Proton Test (APT) and GC-MS werc used to characterize the cycloaddition product. (Figures XLVI-XLIX). ${ }^{1} \mathrm{H}-\mathrm{NMR}\left(300 \mathrm{MHz}, \mathrm{CD}_{2} \mathrm{Cl}_{2} \& \mathrm{CDCl}_{3}\right) \delta(\mathrm{ppm}): 2.4(\mathrm{~s}, 2 \mathrm{H}), 1.2(\mathrm{~m}, 1 \mathrm{H})$, $0.9(\mathrm{~m}, 4 \mathrm{H}), 0.75(\mathrm{~s}, 4 \mathrm{H}), 0.4(\mathrm{~m}, 1 \mathrm{H}) .13 \mathrm{C}-\mathrm{NMR}\left(300 \mathrm{MHz}, \mathrm{CD}_{2} \mathrm{Cl}_{2} \&\right.$ $\left.\mathrm{CDCl}_{3}\right) \delta(\mathrm{ppm}): 114(\mathrm{~s}), 112(\mathrm{~s}), 58(\mathrm{~s}), 50(\mathrm{~s}), 37(\mathrm{t}), 32(\mathrm{~s}), 20(\mathrm{~d}), 6$ $(\mathrm{t}), 4(\mathrm{t})$.

SOLVENT TRAPPING STUDY:

Nishida ${ }^{30}$ suggested that this type of reaction goes through a 1,4-dipolar intermediate as shown below.

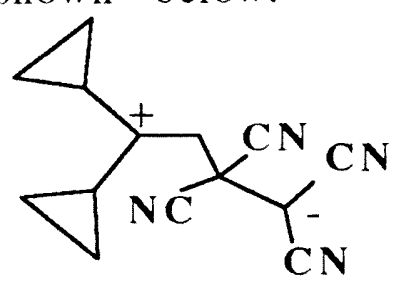


This type of intermediate will stabilize the positive charge by the cyclopropyl groups and the negative charge will be stabilized by the cyano groups. Solvent trapping was attempted at $0{ }^{\circ} \mathrm{C}$ and at $-70{ }^{\circ} \mathrm{C}$, no trapping was observed at either temperature, the intermediate might be too short-lived species to be trapped under our experimental conditions. 
FIGURE XLVI: ${ }^{1}$ II NAIR SPECTRUM OF REACTION OF 1,1-DICYCLOPROPYLETIYLENE WITII TCNE

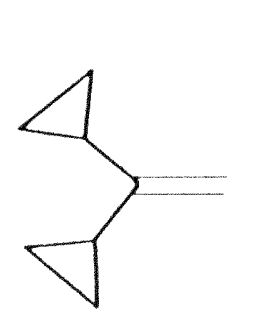

$+$<smiles>[14CH3]C([N])=C(C#N)C#N</smiles>

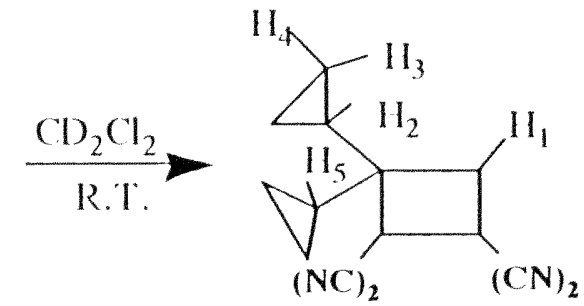

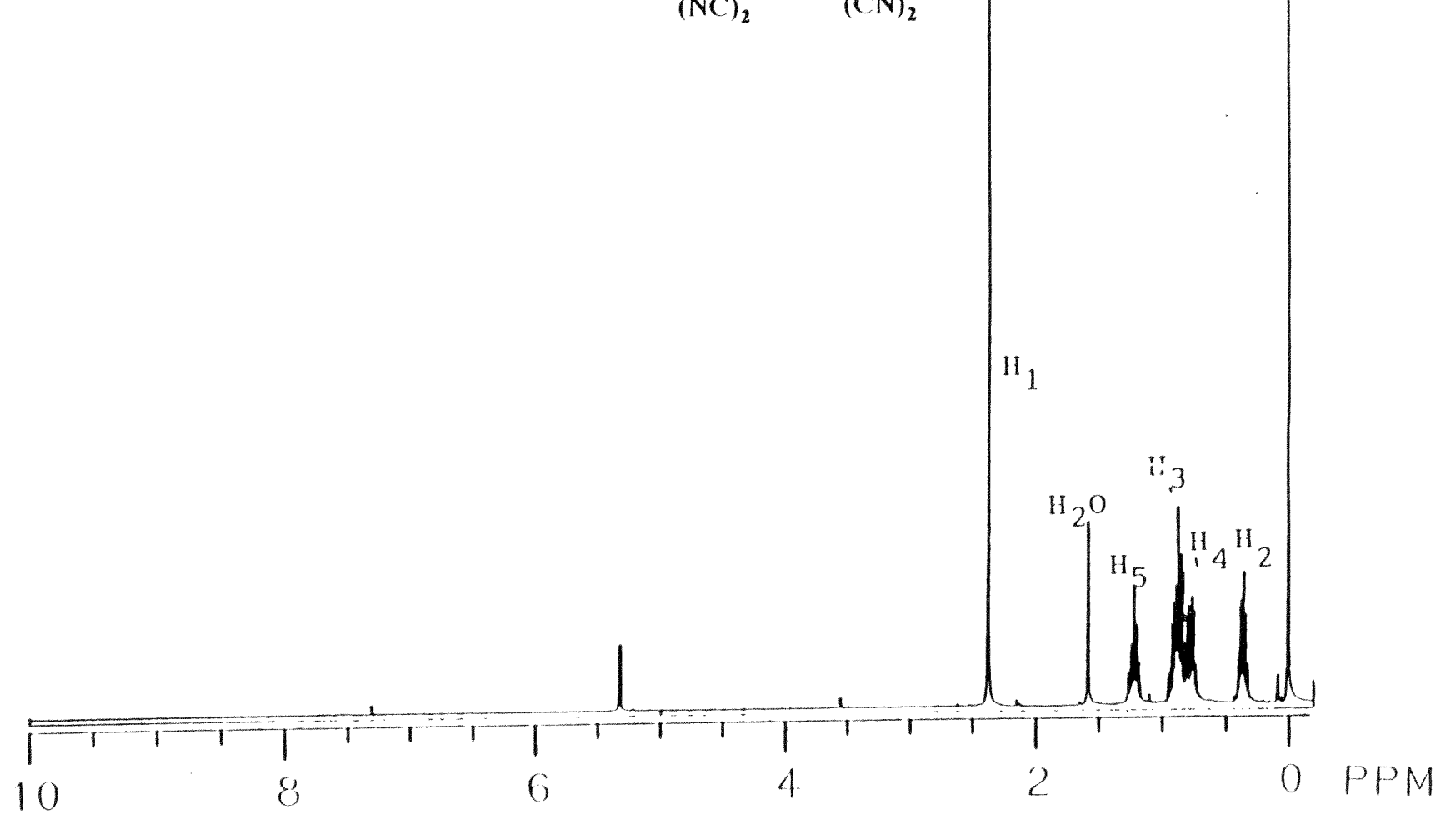




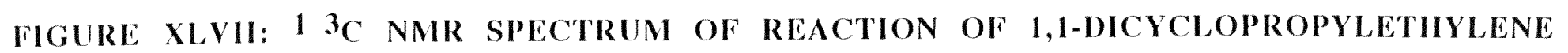
WITII TCNE

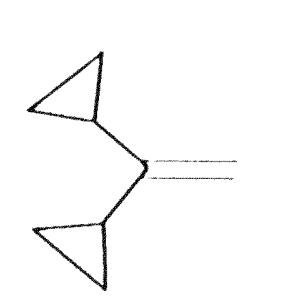

$+$
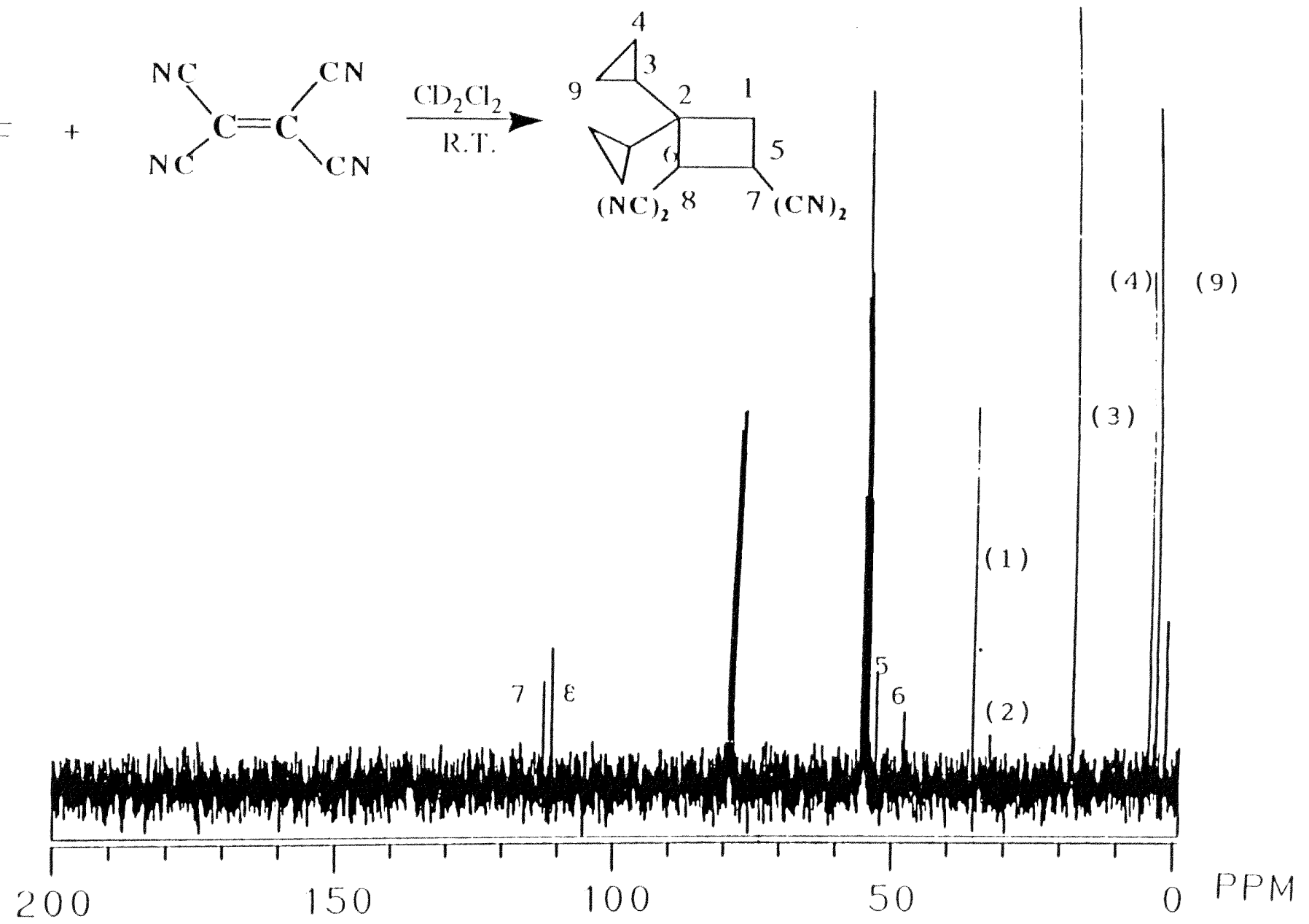
FIGURE XLVIII: ATTACHED PROTON TEST SPECTRUM OF REACTION OF 1,1DICYCLOPROPYLETHYLENE WITH TCNE

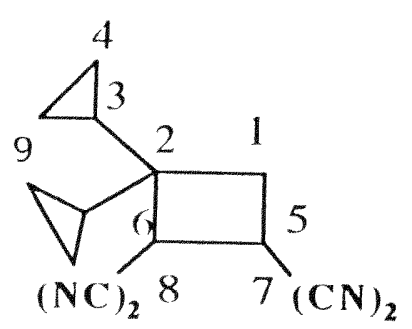

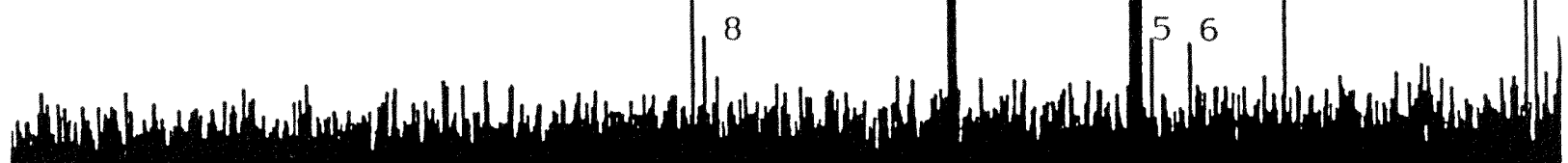

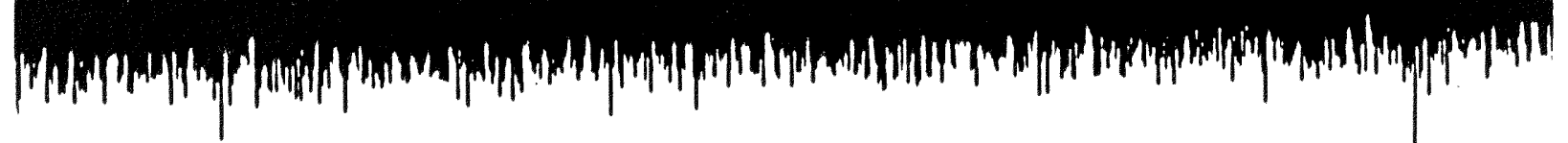

3

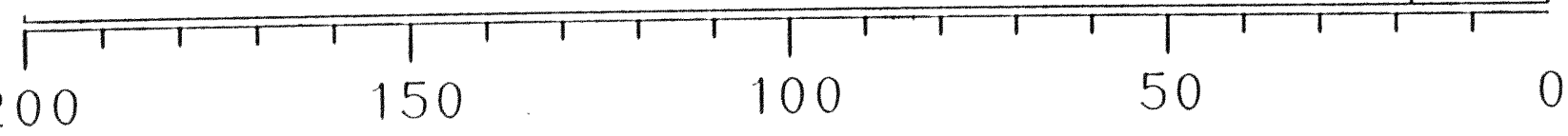


FIGURE XLIX: MASS SPECTRUM OF REACTION OF 1,1-DICYCLOPROPYLETHYLENE
WITH TCNE

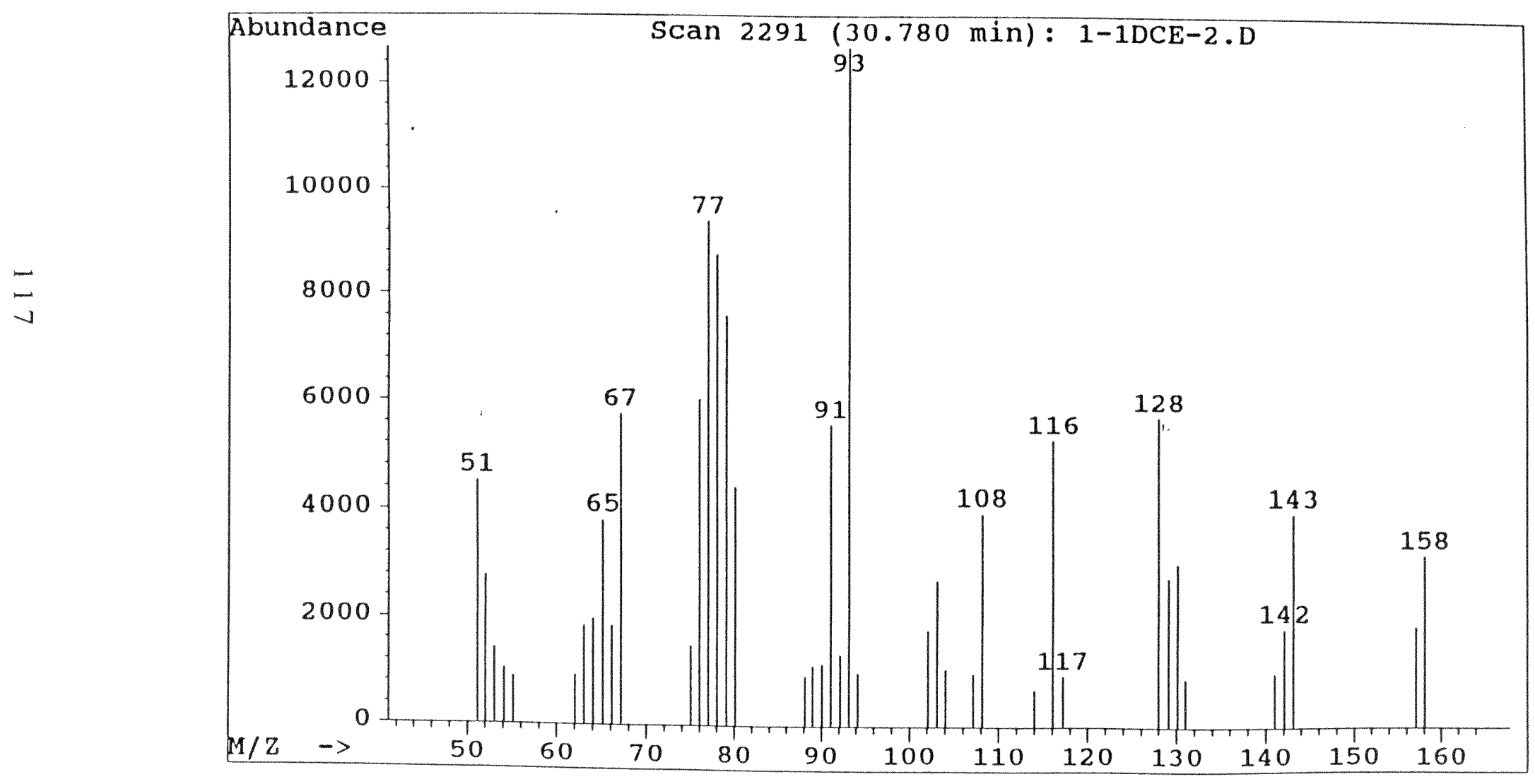




\subsubsection{REACTION OF TETRACYANOETHYLENE (TCNE) WITH $c$ is- DICYCLOPROPYLETHYLENE}

Nishida ${ }^{30}$ observed the lack of reactivity of the cis-and transdicyclopropylethylene with tetracyanoethylene at room temperature. He studied the introduction of a methyl group or a third cyclopropyl group in the structure of disubstituted olefins increases the rate of the reaction. However the ionization potential of the cis- and transdicyclopropyleythylene is lower than that of 1,1dicyclopropylethylene, their reactivity is much lower with respect to TCNE.

\section{RESULTS:}

Twenty microliters of pure cis-1,2-dicyclopropylethylene were mixed with $1 \mathrm{~mL}$ of $\mathrm{CD}_{2} \mathrm{Cl}_{2}$ or acetone, $\mathrm{d}_{6}$ and placed in a NMR tube which was kept in a Dewar containing an ice-water bath until the temperature equilibrated. An equimolar amount of TCNE was then added. After mixing the contents properly, no change was observed for $24 \mathrm{hrs}$. TCNE was added in excess to the reaction solution at room temperature and the reaction mixture was heated at reflux using benzene for $\sim 3$ hrs. Formation of one product was determined by NMR spectroscopy. ${ }^{1} \mathrm{H},{ }^{13} \mathrm{C}$ NMR was used to characterize the final product that was yielded almost quantitatively which corresponds to the cycloaddition product (Figures L-LI). Solvent trapping was attempted using methanol at $0{ }^{\circ} \mathrm{C}$ and at $-70{ }^{\circ} \mathrm{C}$. No reaction was detected at either temperature. 
FIGURE L: 1 II NMR SPECTRUM OF REACTION OF $c$ is-DICYCLOPROPYLETHYLENE WITII TCNE
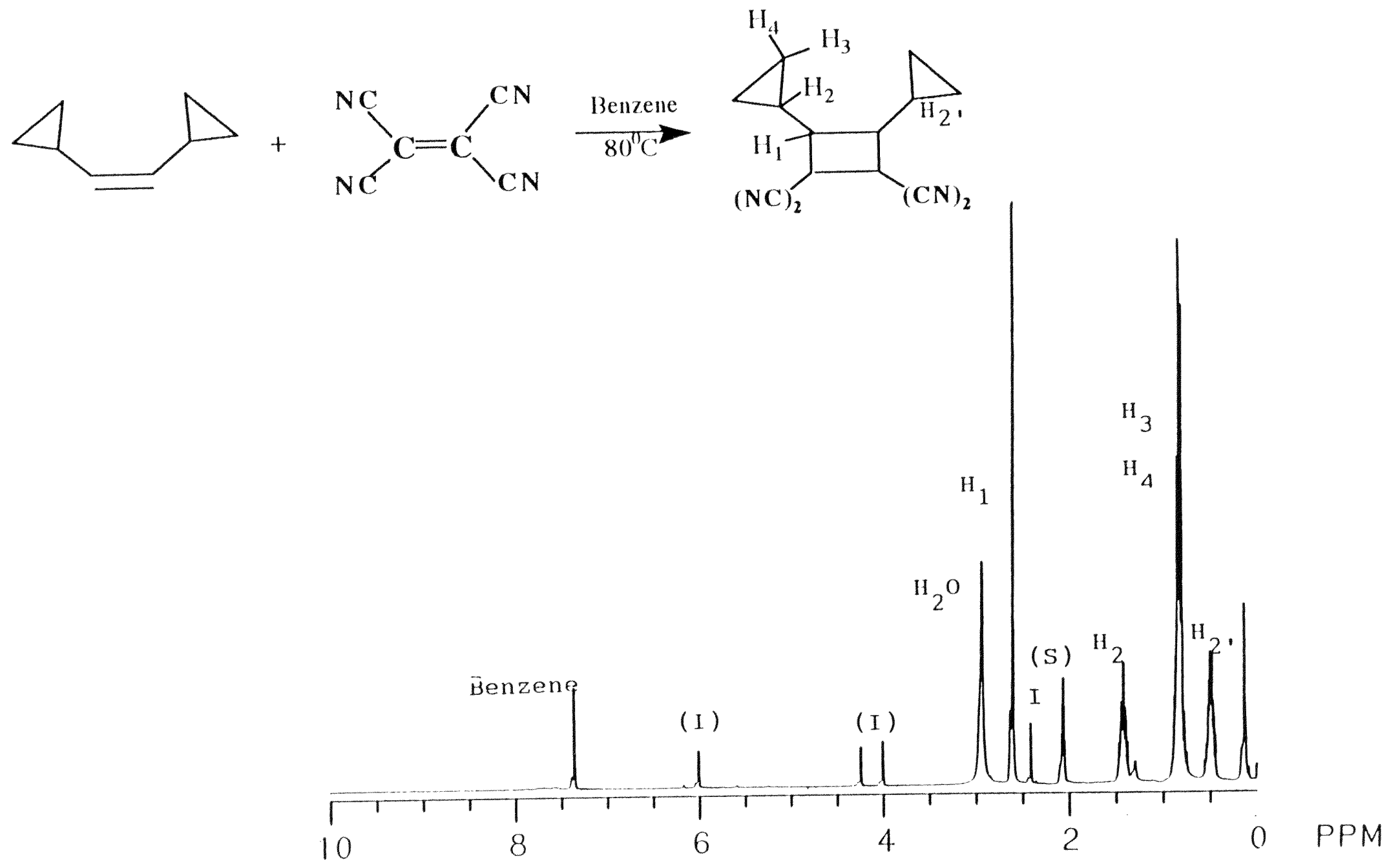
FIGURE LI: $13^{3}$ C NMIR SPECTRUM OF REACTION OF $c$ is-DICYCLOPROPYLETIYLENE IVITII TCNE
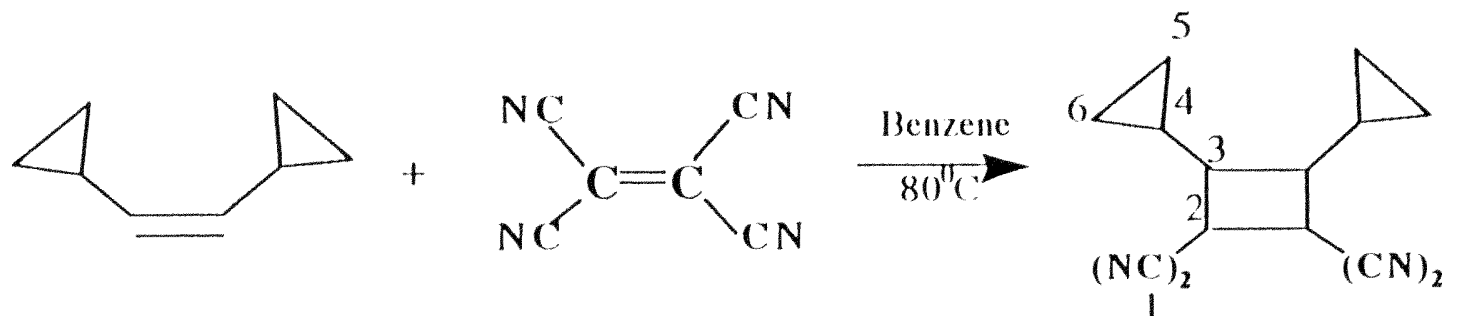

10

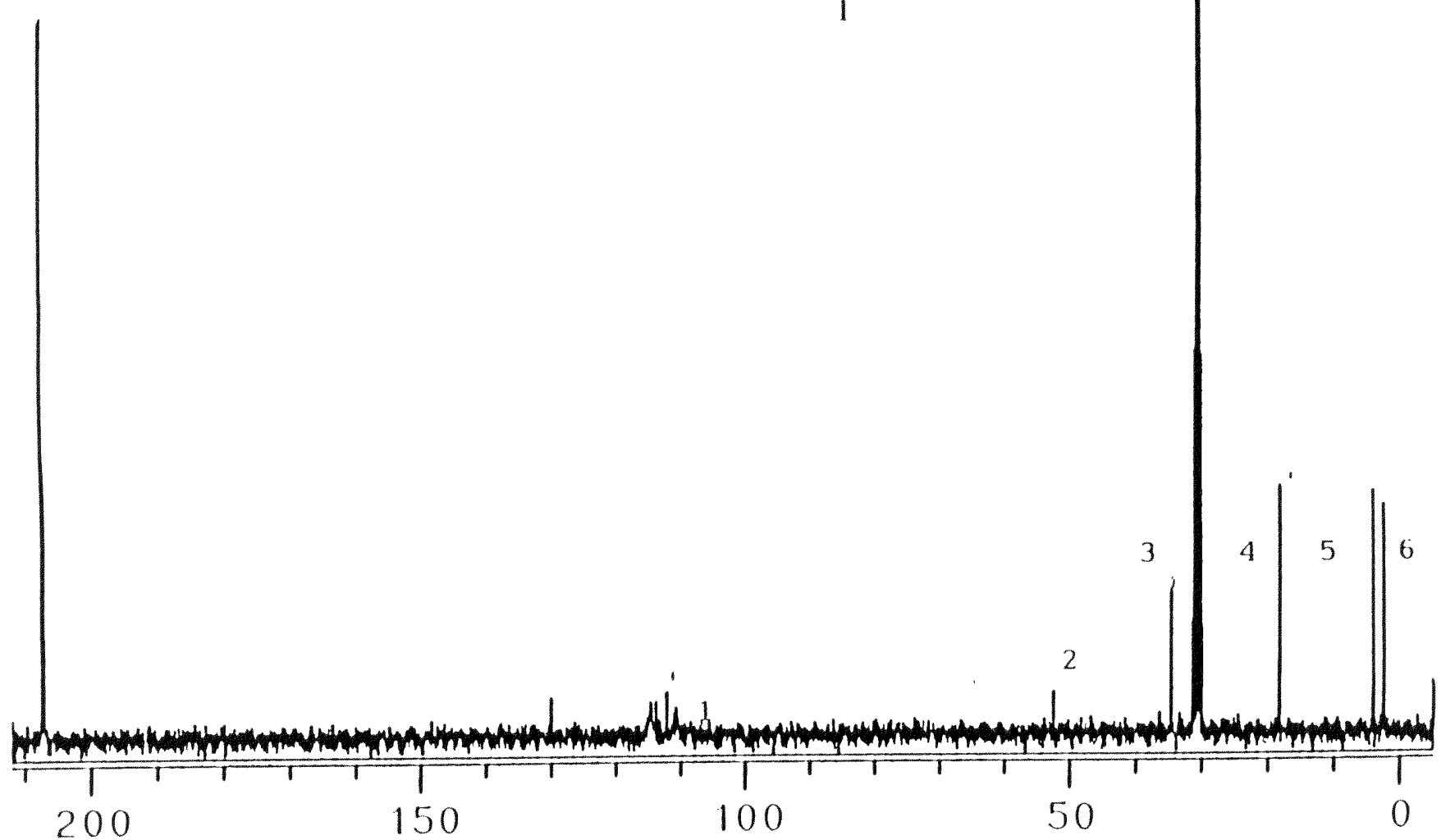




\section{S UMMARY}

Singlet oxygen and singlet oxygen mimics, TCNE and MTAD, exhibit dramatically different reactivitics and reaction pathways. Product studies demonstrated that singlet oxygen does not react with the substrates 1,1-dicyclopropylethylenc and cis-1,2dicyclopropylethylene, in the presence of ZnTPP as sensitizer.

TCNE reacts with 1,1-dicyclopropylethylene and cis-1,2dicyclopropylethylene affording the $2+2$ cycloaddition product. Our results imply a concerted mechanism involved in the reaction.

MTAD reacts with 1,1-dicyclopropylethylene to yield the 2+2 adduct and a product which appears to be a rearrangement of the expected ene product. In the presence of methanol, no $2+2$ or rearrangement products are observed at the expense of a methanol adduct (Markonikov). This suggest a closed zwitterionic type intermediate is involved in the reaction. We were not able to detect any intermediates by NMR at temperatures down $-70^{\circ} \mathrm{C}$.

cis-1,2-Dicyclopropylethylene reacts with MTAD to form 1,2diazetidine in nearly quantitative yield. Product distribution changes dramatically in the presence of methanol, the $2+2$ product is reduced to $25 \%$ with the formation of a methanol adduct $\sim 75 \%$. While the methanol adduct implies the involvement of a zwitterionic intermediate the stereospecificity of the products suggest a closed zwitterion, aziridinium imide, which cannot undergo bond rotation. Therefore we suggest a close zwitterion as the predominant intermediate in the reaction pathways of MTAD with cyclopropylethylenes. 


\section{REFERENCES}

1) Wasscrman, H. H. and Murray, R. W. Singlet Oxygen, Academic Press, New York, 1979.

2) Foote, C. S.; Wexter, S. S. and Ando, W. Chemistry of Singlet Oxygen. III Product selectivity, Tetrahedron Lett. 1965, 4111.

3) Horspool,W. M. and Pill-Soon, Song CRC Handbook of Organic Photochemistry and Photobiology, $1995,1407$.

4) Gollnick, K. Adv. Photochem. $1968,6,1-22$.

5) Foote, C. S. Definition of Type I and Type II photosensitized oxidation, Photochem. and Photobiol. $1991,54,659$,

6) Frimer, A. Singlet Oxygen, Vol II, Reaction modes and products, Part 1, CRC, 1985 .

7) Ohashi, S.; Leong, K. ; Matyjaszewski, K.; Butler, G. B. J. Org. Chem. 198 0, 45, 3467, b) Hoye, T. T.; Bottorff, K.; Caruso, A. J.; Dellaria, J. F. J. Org. Chem. 1980,4 5, 4287.

$8)$ Fenical, W.; Kearns, D. R.; Radlick, P. J. Am. Chem. Soc. 1969 , $91, \quad 7771-7772$.

9) a) Anastassian, A. G. and Yakali, E. J. C. S. Chem. Comm. 1972 , 92. b) Findlay, D. M.; Ray, M. L. and Mc Lear, S. Can. J. Chem. $1972,50,3186$.

10) Burrage, M.; Cookson, R.; Gupte, S.; Stevens, I. J. C. S. Perkins II, $1975,1325$.

11) Yamaguchi, K.; Yabushita, S.; Fueno, T. and Howk, K. N.; On the mechanism of photooxygenation reactions. Computational 
cvidenec against the diradical mechanism of singlet oxygen ene reactions., J. Am. Chem. Soc. $1981,103,5() 43$.

12) Rigandi, J.; Capdevielle, P.; Cambrisson S.; and Manny, M.; Tetrahedron Lett. $1974,2757$.

13) Yamaguchi, K., Theoretical calculation of singlet oxygen reactions, Singlet Oxygen, Frimer, A. Ed. CRC Press, Boca Raton, Fl., Vol III, $1985,119$.

14) Jefford, C. Chemical Society Reviews, $1993,59$.

15) Jensen F.; Foote C. J. Am. Chem Soc. 1987, 109, 6376-6385.

16) O'Shea K.; Foote C. J. Am. Chem. Soc. $1988,110,7167$.

17) Sharp, D. B., Abstracts of paper, 139th meeting of the American Chemical Society, New York, 1960; American Chemical Society; Washington, DC., 1960, 79.

18) Kearns, D. R. J. Am. Chem. Soc. 1969,9 1, 6554-6553.

19) Frimer A. A.; Barlett, P. D.; Boschung, A. F.; Jewet, T. J. G. J. Am. Chem Soc. $1977,99,7977-7986$.

20) Stephenson, L. M. Tetrahedron Lett.1980,21 1005.

21) Greene F.; Seymour C. J. Am. Chem. Soc. $1980,102,6384-$ 6385 .

22) Nelsen, S.; Kapp, D. J. Am. Chem Soc. 1985,107 , 5548-5549.

23 ) Squillacote, M.; Mooney, M.; De Filippis, J. J. Am Chem. Soc. $1990,112,5364-5365$.

$24)$ Orfanopoulos, M.; Smonou, I.; Khan, S.; Foote, C.; Elemes, Y.; Mavridis, I.; Pantidou, A. J. Am. Chem. Soc. $1995,117,7081$ 7087 . 
25) Footc, C.; Poon, T.; Parks, S.; Elemes, Y. J. Am. Chem. Soc. 1995 , $117, \quad 10468-10473$.

26) Fukui, K.; Kagaki, S.; Fujimoto, H. J. Am Chem. Soc. 1975,97 , $7480-7484$.

27) Dewar, M. J. S.; Thicl, W. J. Am. Chem. Soc. 1977 ,99, 23382339.

28) Kearns, D. R. Chem Rev. $1971,71,395$.

29) Harding, L. B.; Goddard, W.A. II; J. Am. Chem. Soc. 1980,102 , $439-449$.

$30)$ Nishida, S.; Moritani, I.; Teraji, T. J. Org. Chem. 197 3, $38,1878$.

$31)$ Tsuji, T.; Nishida, S.; Acc. Chem. Res. 1984,1 7, 56-61.

32) Rappoport, Z. The Chemistry of the Cyclopropyl group; Parts 1 and 2; John Wiley \& Sons Ltd.: New York, 1987. Greenberg, A.; Liebamn, J. F. Strained Organic Molecules ; Academic Press: New York, 1978 .

33 ) Coulson, C. A.; Moffit, W. E. Phil. Mag. $1949,1,40$.

$34)$ Walsh, A. D. Nature London, 194 7, 159, 167, 712. Walsh, A. D.; Trans. Faraday Soc. $1949,45,179$. Sudgen, T. M. Nature (London), $1947,160,367$.

35) Drumright, R. E.; Mas, R. H.; Merola, J. S.; Tanko, J. M. J. Org. Chem. $1990,55,4098-4102$.

36) Jorgensen, W. C.; Salem, L. The Organic Chemist book of Orbitals; Academic Press: New York, 1973.

37 ) a) Hoffman, R. Tetrahedron Lett. 197 0, 2907. b) Hoffman, R.; Davidson R. B. J. Am. Chem. Soc. 197 1, 9 3, 5699. 
38) Clark, T.; Spitnagcl, G. W.; Klose, R.; Schlcyer, P. v. R. J. Am. Chem. Soc. $1984,106,4412$.

39 ) a) Shimazaki, M.; Hara, H.; Suzuki, K.; Tsuchihashi, G.; Tetrahedron Lett. $1987,28,5891$. b) Shimazaki, M.; Hara, H.; Suzuki, K.; Tetrahedron Lett. $1989,30,5443$. c) Shimazaki, M.; Hara, H.; Suzuki, K.; Tetrahedron Lett. 1989,30 , 5447. d) Shimazaki, M.; Moritomo, M.; Suzuki, K.; Tetrahedron Lett. $1990,31,3335$.

40) Shono, T.; Fujita, K.; Kumai, S.; Watanabe, T.; Nishiguchi, I. Tetrahedron Lett. 1972 , 3249, b) Clark, T.; Spitznagel, G. W.; Klose, R.; Schleyer, P. v. R., J. Am. Chem Soc. 198 4, 106, 4412.

$41)$ Cramer, C.; Barrows, S. J. Org. Chem. 1994,59,7591-7593.

42) Olah, G. A. Chemical Reviews, $1992,92,93$

43 ) Greenberg, A.; Liebman, J. F. Strained Organic Molecules; Academic Press: New York, 1978 .

44) Frimer, A. A. J. of Photochem. $1985,25,211-226$.

45) Ketley, A.D.; McClanahan, J. L. Angew. Chem. Int. Ed. 1964,30 , $940-942$.

46) Maercker, A. Angew. Chem. Internat. Ed. $1967,6,557$.

47) Higgins, C.; Baldwin, W. Analytical Chem. 1963 , 473-475.

48) Kobrich, G.; Merkel, D. Angew. Chem. Internat. Ed. 1970 , 9, 243.

49) Kobrich, D. Angew. Chem. Internat. Ed. $1965,4,49$.

50) Texier-Boullet, F.; Foucaud, A. Synthesis $1979,884$.

51 ) Schlosser, M; Christmann, K. F. Angew. Chem. Internat. Ed. $1966,5,126$. 
52) Slaugh, L. H. Tetrahedron $1966,22,1741-1746$.

53 ) Karabatsos, G. J. Am. Chem. Soc. 1960 , 1409-1412.

54) Frimer, A. A.; Roth, D. J. Org. Chem. $1979,44,3882$.

55) Orfanoupolous, M.; Smonou, I.; Foote C. J. Am Chem Soc. 1990 , $112,3607-3614$.

56) Kopccky, K. R.; Reich, H. J. Can. J. Chem. 1973,4 3, 267. 